\title{
Validation of an Experimentally Motivated Predictive Model for the Spontaneous Clustering of Receptors on the Cell Membrane
}

\author{
Emine Guven
}

Follow this and additional works at: https://researchrepository.wvu.edu/etd

\author{
Recommended Citation \\ Guven, Emine, "Validation of an Experimentally Motivated Predictive Model for the Spontaneous \\ Clustering of Receptors on the Cell Membrane" (2015). Graduate Theses, Dissertations, and Problem \\ Reports. 5726. \\ https://researchrepository.wvu.edu/etd/5726
}

This Dissertation is protected by copyright and/or related rights. It has been brought to you by the The Research Repository @ WVU with permission from the rights-holder(s). You are free to use this Dissertation in any way that is permitted by the copyright and related rights legislation that applies to your use. For other uses you must obtain permission from the rights-holder(s) directly, unless additional rights are indicated by a Creative Commons license in the record and/ or on the work itself. This Dissertation has been accepted for inclusion in WVU Graduate Theses, Dissertations, and Problem Reports collection by an authorized administrator of The Research Repository @ WVU. For more information, please contact researchrepository@mail.wvu.edu. 
Validation of an Experimentally Motivated Predictive Model for the Spontaneous Clustering of Receptors on the Cell Membrane

\title{
Emine Güven
}

\author{
Dissertation submitted \\ to the Eberly College of Arts and Sciences \\ at West Virginia University \\ in partial fulfillment of the requirements for the degree of \\ Doctor of Philosophy in \\ Mathematics
}

\author{
Ádam M. Halász, Ph.D, Chair \\ Harvey Diamond, Ph.D \\ Hong Jian Lai, Ph.D \\ Adrian Tudoraşcu, Ph.D \\ Jeremy S. Edwards, Ph.D
}

Department of Mathematics

\section{Morgantown, West Virginia \\ 2015}

Keywords: membrane receptors, hierarchic clustering, VEGF

Copyright (c) 2015 Emine Güven 


\title{
ABSTRACT \\ Validation of an Experimentally Motivated Predictive Model for the Spontaneous Clustering of Receptors on the Cell Membrane
}

\author{
Emine Güven
}

Membrane receptors are specialized membrane-bound proteins that facilitate communication between the intracellular and extracellular membrane. They control signal initiation in many important cellular signaling pathways. Cell signaling (or signal transduction) provides the logical inputs individual cells need in order to perform their role in the context of the organism. Signaling molecules such as hormones, neurotransmitters or growth factors, are secreted by cells in the organism as a result of certain conditions; the cells receiving the signal change (or maintain) their state in response to the signaling input. The incoming information is processed and the response is formulated by a complex bio-molecular network.

For many ligand / receptor families, receptor dimerization or cross-linking is a necessary step for activation, making signaling pathways sensitive to the distribution of receptors in the membrane. Microscopic imaging and modern labeling techniques reveal that certain receptor types tend to co-localize in clusters. The origin of these clusters is not well understood; they are likely not the result of chemical binding, but of a pre-existing micro-domain structure of the membrane. In this work, we analyze a set of micrographs resulting from a study of vascular endothelial growth factor (VEGF) receptor. VEGF is a protein that is involved in the process of the growth and maintenance of blood vessels. The micrographs represent static snapshots of VEGF receptors. They are obtained by fixing the cells from a cell culture, separating their cell membrane, and then labeling the receptors with nano-gold particles. The samples are then imaged by high-resolution transmission electron microscopy (TEM).

The first part of the work presented here consists of characterizing the two dimensional point distirbutions obtained by identifying the location of the labelled receptor particles. We first applied a number of statistical tests used to establish whether the distributions are consistent with random placement, and whether clustering was present. After establishing the presence of clustering in virtually all images, we proceeded to separate the points in each image into clusters using hierarchic distance based clustering. This method relies on a characteristic length scale that is not a priori identified. Building on previous work, we developed a more refined approach to the identification of an optimal length parameter. We implemented this approach to cluster identification as well as a procedure that assigns a geometric shape to each cluster, in computer script that performs all of these analyses for a set of files. Using the analysis pipeline, we processed approximately 80 images 
that were available and summarized a number of image parameters, measures of clustering, as well as distributions of cluster sizes.

The second part of the dissertation aims to develop and validate a stochastic model of clustering, based on the hypothesis of pre-existing domains that have a high affinity for receptors. The proximate objective is to clarify the mechanism behind cluster formation, and in the longer perspective, to estimate the effect on signaling. We showed that the observed particle distribution results were consistent with the random placement of receptors within the clusters and, to a lesser extent, the random placement of the clusters on the cell membrane. We then defined a simple statistical model, based on the pre-existing domain hypothesis, to predict the probability distribution of cluster sizes.

The model parameters can be identified by fitting to the experimentally derived cluster size distributions. Using a Metropolis-Hastings algorithm, we found that the majority of the images (close to $75 \%$ ) could be fit individually. The remaining images exhibited large scale features that were not meant to be captured in the model. The global fit of the 60 images with a single model parameter set was less successful. We obtained better results by separating the images into groups using k-means clustering, and then performing global fits to each group taken separately. The biological significance of these emerging groups is not clear at the moment; however, the process yielded sets of parameter values that can readily be used in dynamical calculations as estimates of the quantitative characteristics of the clustering domains. 


\section{Dedication}

To my chemistry teacher Meryem Bursa for her immeasurable support during my studies in high school and college.

To my children Ada and Fatih, whose love made my desire to finish my PhD every day and night I stayed away from them.

To my husband Ufuk, for taking care of our kids while I was finishing this dissertation.

To all of my friends for all of their support and encouragement. 


\section{Acknowledgements}

First and foremost, I wish to thank my advisor and dissertation chair Dr. Adam Halasz for his patient guidance, encouraging me during my studies as a student, teaching assistant and research assistant. His guidance and professional style will always inspire me as I continue my career.

I would also like to thank Dr. Diamond, for his advice, being my committee member and assistance in keeping my progress on schedule.

I also thank my other committee members, Dr. Lai, Dr. Tudorascu, and Dr. Edwards for their valuable recommendations during this study.

To Dr. Tudorascu for his help and support during my first years as a graduate student in the United States.

I gratefully acknowledge support from NIH grant R01 GM104973 as well as funds from the State of West Virginia.

Finally, I am especially grateful to my family and friends who supported me over the years. 


\section{Contents}

$\begin{array}{ll}\text { Abstract } & \text { ii }\end{array}$

Dedication $\quad$ iv

Acknowledgements $\quad$ v

$\begin{array}{ll}\text { Introduction } & 1\end{array}$

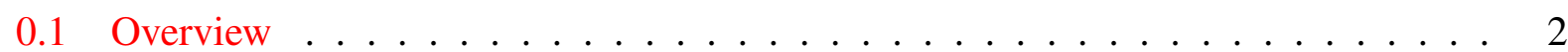

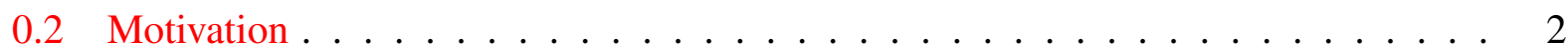

0.3 Biological Background . . . . . . . . . . . . . . . . 3

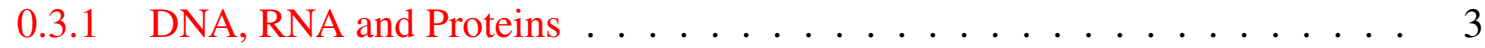

0.3 .2 Cell Signaling and VEGF Receptors . . . . . . . . . . . . . . . 4

1 Data Analysis $\quad 7$

1.1 Experimental Aspects . . . . . . . . . . . . . . . . . . . 7

1.1.1 Source of Data . . . . . . . . . . . . . . . 8

1.1.2 Experimental Methods . . . . . . . . . . . . . . . . . 9 9

1.2 Distributions of Random Geometric Points . . . . . . . . . . . . . . . . . 11

1.2.1 Probability Density Function . . . . . . . . . . . . . . . . . 12

1.2.2 Cumulative Distribution Function . . . . . . . . . . . . . . . 14

1.2.3 Deriving the Nearest Neighbor Probability Distribution Function . . . . . . 16

1.3 Traditional Measures of Clustering . . . . . . . . . . . . . . . . 18

1.3.1 Nearest Neighbor and Next Nearest Neighbor Distance Distributions . . . . 18

1.3.2 The Hopkins Statistic Test . . . . . . . . . . . . . . . . . . . . 19

1.3.3 The Ripley Statistic Test . . . . . . . . . . . . . . . . . . . 21

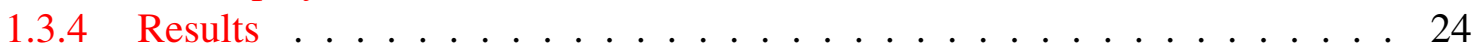

1.4 Hierarchic Clustering . . . . . . . . . . . . . . . . . . . . . . . . . . . . . . . 25

1.4.1 Dependence on Length . . . . . . . . . . . . . . . . . . 28

1.4.2 Identifying the Optimum Length . . . . . . . . . . . . . . . . 28

1.4.3 Domain Reconstruction Algorithm and Cluster Analysis . . . . . . . . . . 31 
2 Model $\quad 36$

2.1 Attractive Micro-domains . . . . . . . . . . . . . . . . 37

2.1.1 A Working Hypothesis for the Mechanism of

Receptor Clustering . . . . . . . . . . . . . . 38

2.1 .2 Model Definition . . . . . . . . . . . . . . . . . . . . 40

2.1.3 Understanding the Spatial Distribution

other than Microdomain-Induced Clustering . . . . . . . . . . . . . . . . . 42

2.1.4 Predicting the Distribution of the

Number of Particles per Cluster . . . . . . . . . . . . . . . . . 43

2.2 Spatial Distribution . . . . . . . . . . . . . . . . . 43

2.2.1 Analysis of Distribution within Clusters . . . . . . . . . . . . . . 43

2.2.2 Analysis of Distribution Between Clusters . . . . . . . . . . . . . . . . 44

2.3 Number of Particles by Cluster . . . . . . . . . . . . . . . . . . . . 45

2.3.1 Distribution of Domain Sizes and Shapes . . . . . . . . . . . . . 45

2.4 Fitting the Stochastic Model of Cluster Distribution . . . . . . . . . . . . . . 49

2.4.1 Model Definition . . . . . . . . . . . . . . . . . . . . 49

2.4.2 Fitting the Model to the Data . . . . . . . . . . . . . . . . . 54

2.4.3 Cluster Number Distributions . . . . . . . . . . . . . . . . 59

$3 \quad$ Discussion and Future Work $\quad 66$

$\begin{array}{ll}\text { Appendix } & \mathbf{6 8}\end{array}$

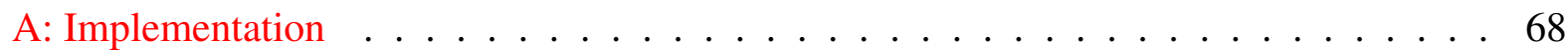

B: Statistical Analysis Program Scripts . . . . . . . . . . . . . . . . 68

C: Creating Figures from Structure . . . . . . . . . . . . . 85 


\section{List of Figures}

1 Genetic code ............................ 4

2 "Central Dogma" of molecular biology . . . . . . . . . . . . . . 4

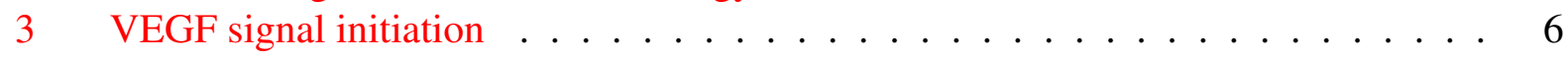

4 Sample TEM image . . . . . . . . . . . . . . . . . . . . . 9

5 Membrane sheet preparation . . . . . . . . . . . . . . . 10

6 High resolution TEM images and particle maps . . . . . . . . . . . . . . . 13

7 Deriving of the nearest neighbor distance distribution . . . . . . . . . . . 17

8 Nearest and next-nearest neighbor distributions from TEM images . . . . . . . . 20

9 Hopkins statistic of selected images . . . . . . . . . . . . . . . . . . . . . . . . 22

10 Hopkins and Ripley statistic of image 5-16616 . . . . . . . . . . . . . . . 23

11 Ripley's K and L for selected images . . . . . . . . . . . . . . . . . . . 24

12 Hopkins statistic for selected images . . . . . . . . . . . . . . . . . . 24

13 Distributions of charateristic parameters by data set . . . . . . . . . . . 26

14 Clustering measures for image $5-16616 \ldots \ldots$. . . . . . . . . . . . 27

15 Number of clusters $\left(N_{C}\right)$ versus the length parameter $(L)$ for random points . . . . 29

16 Short distance NND and $N_{C}(L)$ are consistent with random placement of points . . 30

17 Estimating the optimal $L$ from the inflection point of $N_{C}(L) \ldots \ldots$. . . . . 30

18 Cluster assignments for image 5-16616 using different $L$ values . . . . . . . . . . 32

19 Algorithmic identification of $L$ using the inflection point . . . . . . . . . . . . 33

20 Work flow for identifying a geometric shape containing a cluster . . . . . . . . . 33

21 Contour building algorithm . . . . . . . . . . . . . . . 35

22 Apparent microdomains in image 5-16622 . . . . . . . . . . . . . . . . . 37

23 Cluster identification and sizes in image $5-16626 \ldots \ldots$. . . . . . . . . . . . . . . . . . . . . . . . . . . . .

24 Physical domains to model domains . . . . . . . . . . . . . . . . . 39

25 Microdomains to compartments . . . . . . . . . . . . . . . . . 41

26 Possible microdomains in image $5-16624 \ldots \ldots \ldots \ldots$. . . . . . . . . . . . . . . . . . . . . . . . . . . .

27 Nearest neighbor distribution for image $5-16616 \mathrm{~b} \ldots \ldots \ldots \ldots$. . . . . . . . . . . . . . . . 42

28 Cluster identification and contours for image $5-16646 \ldots$. . . . . . . . . . . . . . . . . . . . . . . .

29 Cumulative vs. individual clustering measures . . . . . . . . . . . . . . . 45

30 Localization of cluster centers . . . . . . . . . . . . . . . . . . 46 
31 Scatter plots of clustering results . . . . . . . . . . . . . . . 48

32 Histograms of all the clustering information . . . . . . . . . . . . . . 49

33 Scatter plots of model fitting with k-means . . . . . . . . . . . . . . . . . 60

34 Cumulative cluster scaling for two different data set . . . . . . . . . . . . . . 61

35 Histograms of all the model prediction unknowns . . . . . . . . . . . . . . 61

36 Histograms of all the model calculations . . . . . . . . . . . . . . . 62

37 Global fit file by file results . . . . . . . . . . . . . . . . . . . 63

38 Scatter plots of some model parameters by time and antibody type . . . . . . . . . 64

39 Scatter plots of more model parameters by time and antibody type . . . . . . . . 65 


\section{List of Tables}

1 Experimental data sets $\ldots \ldots \ldots \ldots \ldots \ldots$

2 Characteristics of the five data sets . . . . . . . . . . . 25

3 Individual fit parameter results . . . . . . . . . . . . . . 58

4 Model fitting results with k-means . . . . . . . . . . . . . . 59 


\section{Introduction}

The subject addressed in this dissertation arose from an interdisciplinary research project being done with expertise in applied mathematics and computational biology in this department, in collaboration with researchers from Center for Spatio Temporal Modeling of Cell Signaling (STMC) at the University of New Mexico. The main goal of this project is to understand how living cells communicate with the external world. In general, our goal is observing and understanding how the spatial and static organization of vascular endothelial growth factor (VEGF) and related receptors (VEGFR) change during signaling. The work in this dissertation relies on experimental data graciously provided by the Cell Pathology Lab led by Prof. Bridget Wilson, at the University of New Mexico Health Science Center in Albuquerque, NM. The data have been generated on the spatial organization of the molecules by labeling them with nano-gold particles and applying imaging techniques on the particles (receptors) by using high resolution transmission electron microscopy (TEM).

In this study, porcine aortic endothelial (PAE) cells over-expressing KDR (PAE/KDR) were used which is PAE-KDR grown in cultures that stimulated with VEGF for different time scales whereas some cells were not stimulated (resting). The main goal of this dissertation is to describe and quantify the distribution of receptors, in order to facilitate the study of changes during signaling. In the remainder of this chapter, we explain the biological background and fundamentals of cell membrane related to cell signaling and how cell signaling transduction occurs. In Chapter 2, we give an overview of the distribution of random particles which represent receptors. In Chapter 3 , we use a hierarchical clustering algorithm to quantify clustering previously developed by Espinoza and her co-workers. In Chapter 4, we fit experimental data to the model and discuss the results presented here to understand the role of clustering on cell signaling. We will then discuss the possible future work that can be done to improve and relate the work with dynamic properties of VEGF receptor clustering. The Matlab scripts developed for the project are included in the Appendix. 


\subsection{Overview}

In this project, we focus on VEGF receptors. We analyze sets of TEM (transmission electron microscopy) images of membrane sheets of PAE-KDR cells, where VEGF receptors were labeled with immune-gold particles of $6 \mathrm{~nm}$ in diameter. The distribution of membrane receptors on cell surface is mostly heterogeneous since receptors usually form clusters on the membrane surface [25]. High resolution experiments using microscopic imaging and modern labeling techniques reveal that certain receptor types tend to co-localize in clusters, ranging from a few to hundreds of members [16]. Static images of receptors, even in the absence of ligand, typically reveal a clustering pattern. The simplest explanation would be that the receptors form multi-molecular aggregates through cross-linking. However, clustering is observed for VEGF receptors, as well as for other receptor types for which there is no evidence of a binding mechanism leading to aggregates that contain more than two receptors. Our working hypothesis is that some of the micro-domains found on the cell membrane have a specific molecular composition that results in an affinity for the receptors; receptors may diffuse in and out of the domain boundaries, but the crossing probability is asymmetric.

VEGF mediated signaling [8] is involved in angiogenesis and is important in normal development, as well as in conditions including wound healing, diabetes and cancer. For various cancers, several clinical trials are testing the efficacy of anti-VEGF/VEGF receptor therapies [2]. VEGF receptors share many properties of other receptor tyrosine kinases. Similarly to VEGF receptors, they form ligand-bound dimers in order to activate their intracellular tyrosine kinase domains [14]. Irrespective of the mechanism that causes the clusters, concentrating the receptors in small areas should result in higher dimerization rates, with consequences on signal initiation.

To investigate the observation made from the experiments on VEGF receptors, we have developed a simple spatial stochastic model so that we can describe the characteristics of clusters quantitatively. This model will be able to resolve the distribution of intra-cluster receptors and inter-clusters. The overall goal of this work is to investigate the mechanism that causes the observed clustering and the possible effect of this phenomenon on signaling.

\subsection{Motivation}

The cell membrane is a live selective barrier that separates intracellular components from the extracellular environment. Membrane receptors, or cell surface receptors, are specialized membranebound proteins that facilitate communication between the intracellular and extracellular membrane. Bio-chemical receptors bind very selectively to one (or possibly small number of) molecular species, called ligands. Ligand-receptor binding is one of the elementary processes that facilitate 
cell signaling. Cellular signaling (or signal transduction) is mediated by interactions of proteins. As those proteins become activated, and start signal cascades, cells receive information from their neighbors through a family of proteins known as receptors. Membrane bound receptors physically straddle the cell membrane. Ligand binding ultimately results in changes of the chemical state of the intracellular part (domain) of the receptor molecule. This is the starting point in a chain of events that carries the information (about the presence of the ligand) into the signal-processing network of the cell.

As for many biological processes, the proper integration of signals is critically important. The membrane receptor is degraded with changes in the genes that encode and regulate the receptor protein. Disordered receptor function is involved in several diseases. When the membrane receptors become deficient, the signal transduction can be hindered, which then cause diseases such as asthma, allergies, and cancer. Therefore, cellular proteins on the cell membrane interaction have been studied extensively. Important protein systems include the VEGF (vascular endothelial growth factor) family, which is implicated in various forms of cancer [27, 28] where the VEGF receptor known for its role in cancers.

\subsection{Biological Background}

\subsubsection{DNA, RNA and Proteins}

Deoxyribonucleic acid (DNA) is a molecule that contains the genetic information the cell requires to develop, maintain and reproduce life essential organisms and also in many viruses. DNA is a nucleic acid located in the cell nucleus which is called nuclear DNA, and the mitochondria also has a small amount of DNA called mitochondrial DNA (mDNA). Watson-Crick discovered the very well-known current structure of DNA in 1953. The DNA is made up of a list of codes that has four chemical bases: adenine $(A)$, guanine $(G)$, and cytosine $(C)$, thymine $(T)$. The famous doublehelix structure of DNA consists of two strands that look like a twisted ladder. Each strand pair is made of deoxyribose and phosphate groups. In Figure 1 there is an illustration of how chemical codes bond to form the bases where adenine forms a base pair with thymine, and cytosine forms a base pair with guanine. The DNA is very highly significant because of carrying all the instruction for cells to produce a particular protein. DNA can replicate, or make copies of itself by using patterns of a strand for duplicating the sequence of bases in the double helix. This is a significant property of DNA when cells divide and generate new cells that supply an exact copy of the DNA that represents the old copy of the cell.

Like DNA, Ribonucleic Acid (RNA) is also a nucleic acid along with proteins and carbohydrates whereas it is more often formed as a single-strand folded onto itself. DNA has thymine (T), 


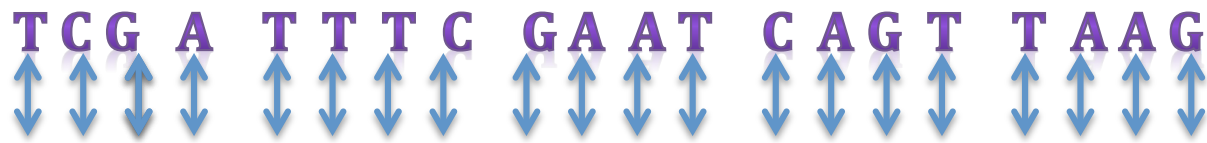 AGC T AA AG C T T G G T C A ATT C}

Figure 1: Main strand and its complimentary strand of a sample genetic code.

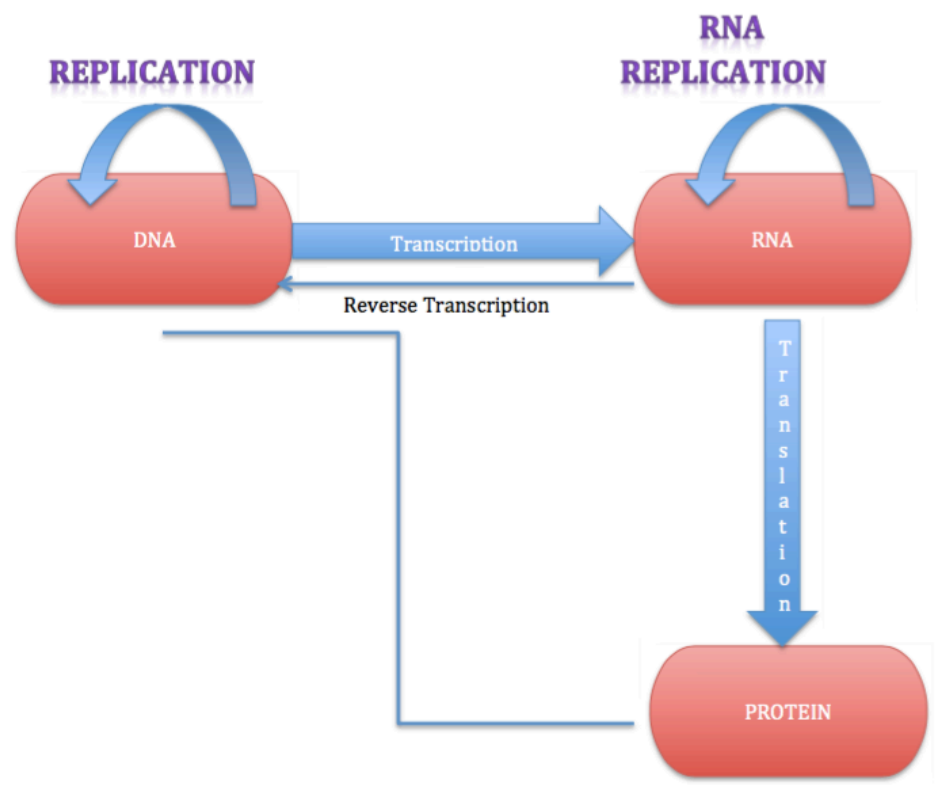

Figure 2: Flowchart of the central dogma of life.

whereas RNA has uracil $(U)$. RNA nucleotides include sugar ribose rather than the deoxyribose. RNA plays roles such as in coding, decoding, regulation and expression of the genes. DNA maintains the protein-encoding information; on the other hand RNA uses that information to enable it to synthesize the particular protein. That information encoded in the sequence of RNA, translates a genes message into a proteins amino acid sequence. The property is determined by protein. This process is called the central dogma of life which is shown as a cartoon illustration in Fig.2.

\subsubsection{Cell Signaling and VEGF Receptors}

Ligand is a generic term for a protein that binds to a receptor and maintains the function on the intracellular domain of the membrane. Cell signaling occurs in response to a ligand that binds to a specific receptor causing a set of changes inside the cell. By signaling, the message moves inside of the cytoplasm of a cell. Functionally these changes can lead to muscle contraction, changes in 
transcription or translation and protein activity, and apoptosis or cell division. Cell signaling which is also known as signal transduction mediates hormones, neurotransmitters, and environmental changes. Many ligands cannot enter the cytosol due to their size or polarity and are unable to cross the lipid bilayer of the plasma membrane. An abnormal deviation in signal transduction has been implicated in several pathologies such as allergies, asthma, and cancer. Therefore, signal transduction pathways have been found interesting, and there are a lot of drugs on the market to target them $[1,6,7,10]$. Because of the experimental innovations and imaging technologies, the knowledge of plasma membrane structure and cellular signaling continues to grow [15, 30, 31].

Vascular endothelial growth factors (VEGFs) are master regulators of vascular development during embryogenesis (vasculogenesis) as well as blood-vessel formation (angiogenesis) in the adult. In mammals, five VEGF ligands have been identified that take roles in several different splice variants and processed forms. VEGF receptor -1, -2 and -3 (VEGFR123) bind in a way that have overlapped patterns to three receptor tyrosine kinases (RTKs). In certain respects, VEGFs share similar regulatory mechanisms with other RTKs, such as the epidermal growth-factor receptors (EGFRs). These mechanisms include receptor dimerization and activation of the tyrosine kinase, as well as creation of docking sites for signal transduction. The VEGFRs also seem to be unique when it compared with other RTK families in terms of their ability to transduce signals that form the three-dimensional vascular tube, and in regulating vascular permeability that leads to oedema and swelling of tissues. As it is demonstrated in Fig.3, mammalian vascular endothelial growth factors (VEGFs) bind to the three VEGF receptor (VEGFR) tyrosine kinases that form VEGFR homodimers and heterodimers. Moreover, VEGFR signaling is modulated by different co-receptors and VEGFs as well as VEGFRs bind to co-receptors. These protein interactions can affect VEGFR-mediated responses, such as affecting the half-life of the receptor complex [20].

The motivation of this project is coming from the considerable evidence that the cell membrane is separated into isolated compartments, or regions, which we call micro-domains, such as lipid rafts [19] and protein islands [33]. The formation of clusters and their role in cell signaling has a great impact in promoting studies in the roles of membrane micro-domains in signal initiation through computational methods [24]. There is general agreement that the composition of these micro-domains is heterogeneous. Furthermore, micro-domain stability on the membrane cytoskeleton is influenced by membrane proteins and lipids. These interactions form picket fences and corrals which limit the diffusion of membrane elements of the cytoskeleton [29, 11].

Our starting point to quantifying VEGF receptor localization and clustering is the work of Espinoza and co-workers [5], who initially developed some of the methods for the analysis of nano-gold labelled membrane proteins. Their method provides a number to identify clusters and compare the extent of clustering between experimental conditions where the hierarchical clustering approach is used to compute a hierarchy of clusters that depend on a clustering distance $d$. Similar to their work on hierarchical distance-based clustering, the first objective is to derive a natural length scale. If the particles are grouped into naturally defined clusters (in the sense of a clear scale 


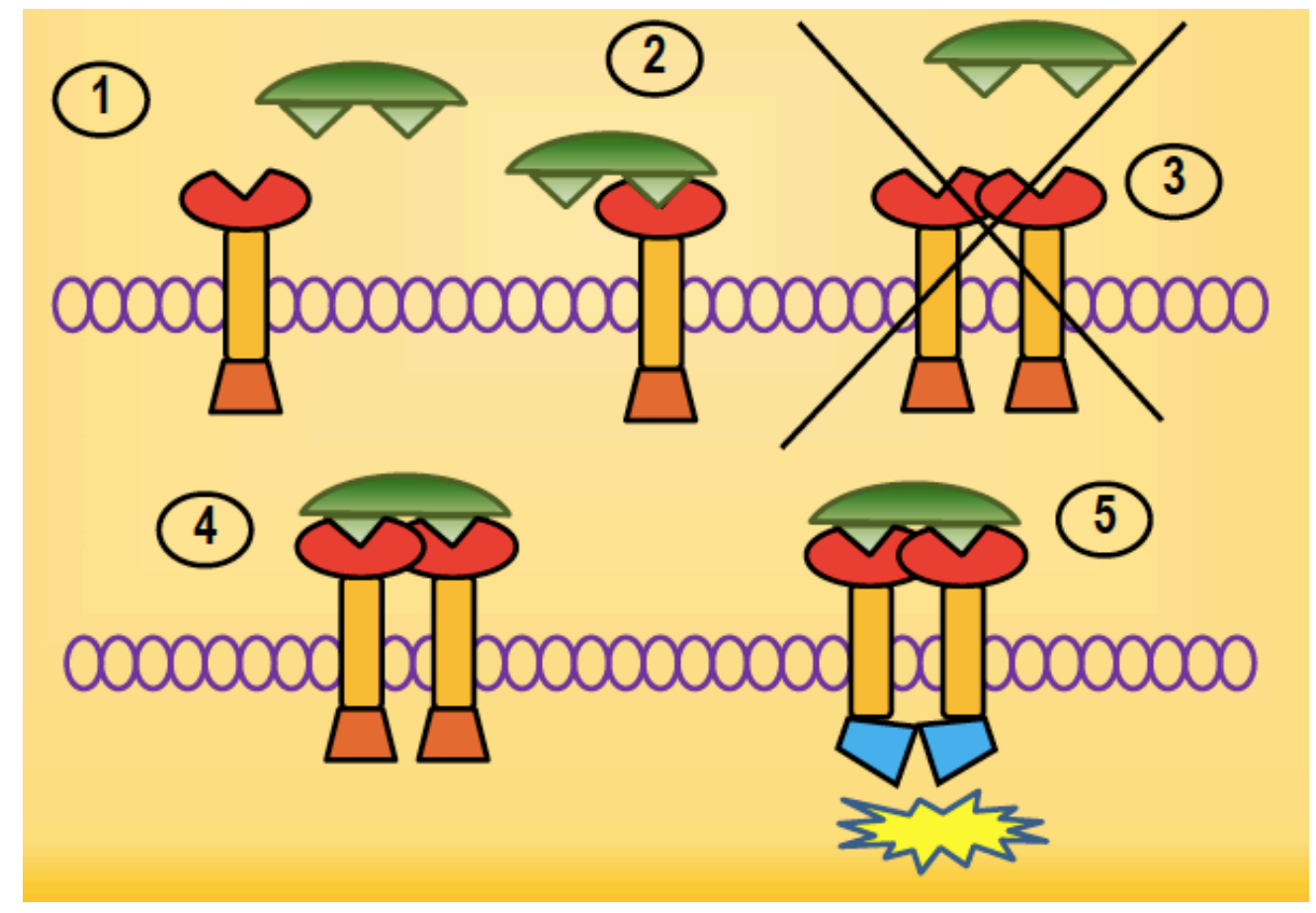

Figure 3: Steps leading up to VEGF signal initiation. Ligand binding (2) is thought to be necessary for dimerization (4), which is required for the mutual activation of the receptors (5). Ligand-independent dimerization (3) may be possible, but that is likely to have a limited role, since would allow signaling in the absence of ligand.

separation between intra- and inter-cluster distances), this natural length scale can be interpreted either as the typical, or the largest intra-cluster distance between a particle and its nearest neighbor. The second definition seems more practical since this would result in correctly separating the particles into clusters. A precise mathematical definition is the necessary step to the point of how to choose this distance. We start with visualizing the distribution of clusters for various length scales to observe dependency on length when we apply distance based hierarchic clustering. Moreover, a reliable and rational definition of clusters that properly conveys the subjective notion, and can be compared with model predictions, requires careful mathematical construction. 


\section{Chapter 1}

\section{Data Analysis}

This section is devoted to the direct characterization of the experimental data, focusing on an objective and methodical description of the observations, avoiding an explicit hypothesis that would explain the observations.

In the first subsection we briefly describe the experimental conditions and the methods used to obtain the TEM images, as well as the extraction of coordinate information from them. Examples of nearest neighbor distribution probability in one and two dimensional spaces are discussed along with the calculation of probability density and cumulative distribution functions. Nearest neighbor distance distribution for several particles are derived and used to analyze the localization of particles (receptors). The mathematical tools for understanding spatial organization are spatial statistics and cluster analysis. Static data has been previously studied using some of these methods, to better understand the spatial organization of molecules on the cell membrane [22, 21, 23, 34]. One of the tools to understand the resulting patterns is the Hopkins statistic, which distinguishes non-clustered from modestly and highly clustered distributions. Hopkins and Ripley statistic tests are used to characterize the distributions of receptors in resting and activated cells. These statistics are given by a plot of the statistic for simulated random data to be compared with a plot of the statistic computed from the experimental data where these methods can distinguish between more and less clustered data.

\subsection{Experimental Aspects}

Cells communicate with the outside world through membrane receptors. A receptor can recognize one of many possible stimuli such as hormones, antibodies, peptides, and other cells in the ex- 
tracellular environment, and translate this input to intracellular responses ("output"). The process of signaling is potentially affected by changes in the organization and composition of the plasma membrane [17], it therefore is very important and interesting to understand the organization of membrane proteins, both in resting cells and activated cells, by tracking their dynamic features during signaling [12]. Experimental investigation over the last couple of decades has focused on the process of binding of ligand to cell surface receptors. Dynamic aspects of these phenomena have attracted more interest following early work of equilibrium binding properties [13].

\subsubsection{Source of Data}

The data we analyze here is a set of transmission electron microscopy (TEM) micrographs obtained in the Cell Pathology Lab led by Prof. Bridget Wilson, at the University of New Mexico Health Science Center in Albuquerque, NM. Porcine aortic endothelial cells expressing human VEGFR2 (PAE-KDR) grown in cultures were prepared by separating their cell membrane and labeling VEGF receptors therein with metal beads a few nanometers in diameter. These beads appear as dark dots in the TEM micrographs. The images provide information on the localization of VEGF receptors. The primary goal of the experiments, and of this dissertation, is to characterize and understand the distribution pattern that was observed. For this purpose, the location of the labelled receptors (identified as dark spots) in each micrograph was recorded in a two-dimensional coordinate system (see Fig.4). The object of our analysis are the sets of geometric points obtained in this manner.

\begin{tabular}{|l||l|l|l|}
\hline \multicolumn{4}{|c|}{ Experimental Data Sets } \\
\hline Condition & $\begin{array}{l}\text { Number } \\
\text { of images }\end{array}$ & \multicolumn{2}{|c|}{ Number of Receptors } \\
\cline { 3 - 4 } & (total) & (average) \\
\hline Abcam 0 min & 21 & 2625 & 125 \\
Abcam 2 min & 14 & 2457 & 175 \\
Abcam 5 min & 29 & 3930 & 135.5 \\
CS 0 min & 8 & 1015 & 126.8 \\
CS 2 min & 10 & 935 & 93.5 \\
\hline
\end{tabular}

Table 1: List of experimental data sets. Condition refers to the antibody used for labelling (Abcam or $\mathrm{CS}$ ), and the time cells were exposed to VEGF before sampling. Each condition resulted in a set of high definition (25000x magnification) micrographs that were processed through the data analysis pipeline presented here. Columns 3 and 4 indicate the number of receptors (also referred to as points or particles) that were identified in the respective set of images. 

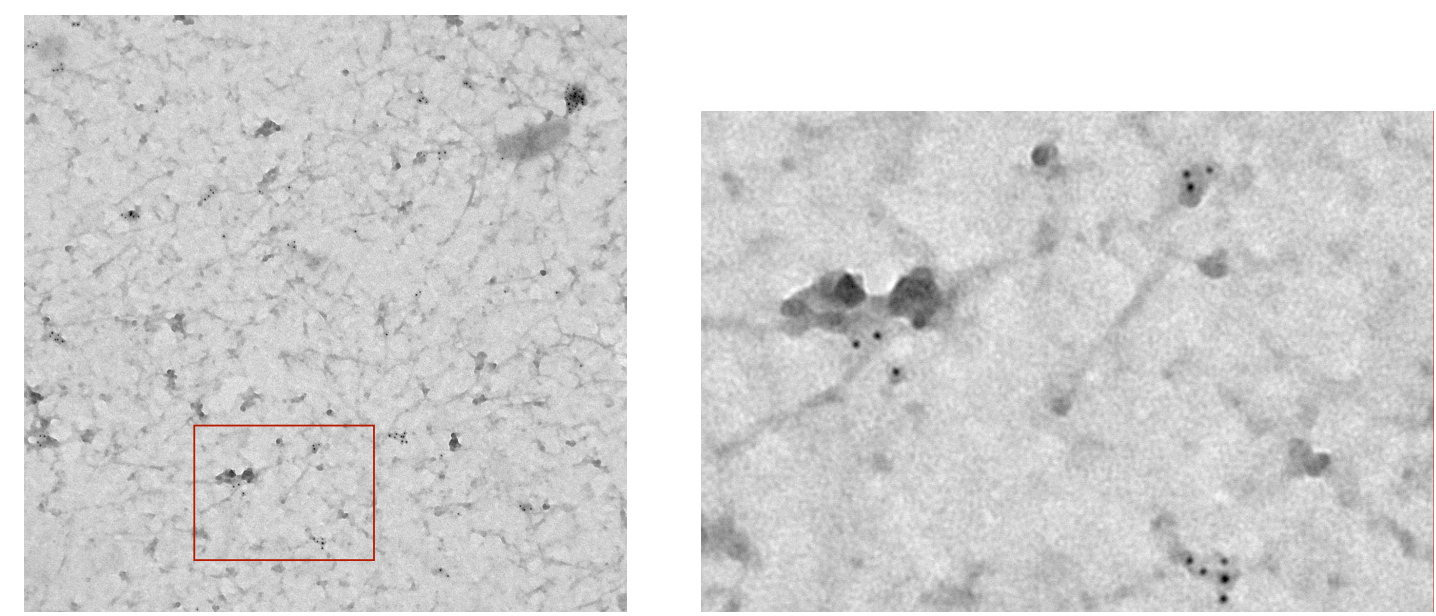

Figure 4: Our data consists of transmission electron microscopy (TEM) images of nano-gold labelled VEGF receptors on the membrane of PAE-KDR cells, courtesy of the Wilson lab at UNM. The images shown are details of the same image to its left. The gold particles appear as dark spots, whose coordinates are extracted in a semi-automatic procedure.

\subsubsection{Experimental Methods}

Transmission electron microscopy (TEM) provides high resolution information about the spatial organization of membranes. Labeling of membrane components by using electron-dense probes results in microgaphs that have details on the level of individual molecules.

The cells used in this experiment were stimulated with VEGF for selected times. Samples were prepared by rapidly ripping and fixing membrane sheets (see diagram, Fig.5), the cytoplasmic face, then the cytoplasmic tails of specific proteins are labeled of specific gold nanoparticles[32]. Membrane sheets are labeled with electron probes (10 $\mathrm{nm}$ gold nanoparticles). Labelled samples were then imaged by transmission electron microscopy. The gold nanoparticles used for labeling appear as dark spots on the resulting micrographs.

The micrographs use the same magnification (1:25000); each micrograph has $2650 \times 2650$ pixels with approximately $1.44 \mu \mathrm{m}$. This corresponds to a square field of view of approximately $1.44 \mu \mathrm{m}$, or an area of approximately $14.7 \mu \mathrm{m}^{2}$. In a typical micrograph, we identified around 100 to 200 receptors; outliers range from approximately 50 to approximately 1500 . The complete prepared membrane sheets are approximately $20 \times 20$ microns, so we estimate the total area of the cell membranes around $1000 \mu \mathrm{m}^{2}$. According to the vendor, the PAE/KDR cells express approximately $10^{5}$ receptors, which should work out to approximately 1500 receptors on average in an approximately $15 \mu \mathrm{m} \mathrm{m}^{2}$ area. This would imply that the labeling efficiency is no more than 10 

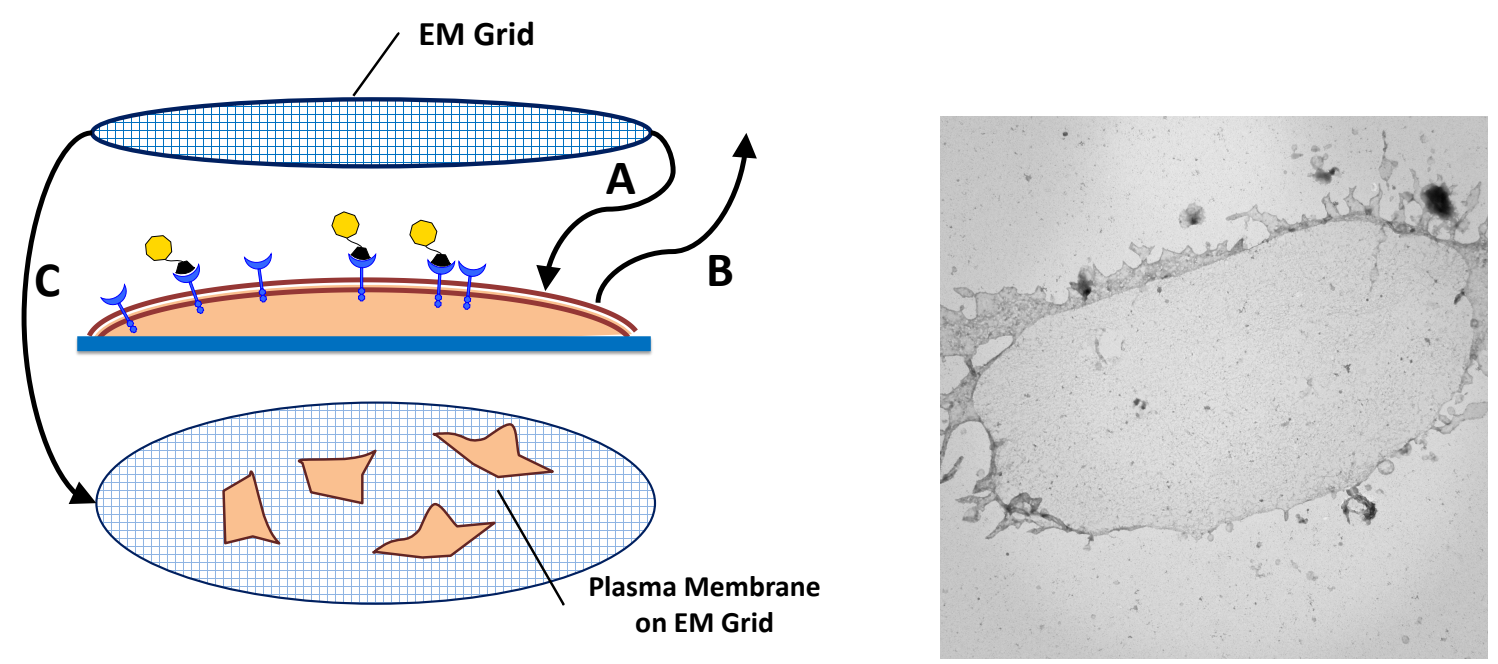

Figure 5: Left: Preparation of plasma membrane samples, following [35]. (A) Lower the EM grid onto the cell on the coverslip, (B) remove the EM grid to produce fragments of the plasma membrane on the grid (C). Right: TEM image of a large membrane sheet fragment.

pixels; it is also likely that the expression level was significantly lower than what was claimed.

In this project, we study TEM images of PAE-KDR cells, porcine aortic endothelial cells that artificially express VEGFR-2 (KDR) receptors. The receptors are labelled with $10 \mathrm{~nm}$ diameter gold particles [35]. Here, each labelled receptor is represented by a dark spot (see Fig.4). The locations of the spots are used to determine the location of receptors with high precision. Sources of uncertainty include the size of the probe and that of the molecule. The images should be interpreted as snapshots of the position of the receptors, at the moment when the cell was prepared for imaging.

We use ImageJ [26] with the ParticlePicker plugin and the CoLocalization plugin. We copy these plugins to the plugins directory of ImageJ (preferably, create separate folders under plugins to hold each file). After starting the ImageJ software, we use "Plugins Compile and Run Plugin" to compile these two programs to extract the receptor coordinates location in two dimensional space. Cropping of the image is done by hand to enable finding the correct location of each receptor. By adjusting the adding and removing probes on the data, we handle the missing particles. Then we export the resulted file into a text document to plot the receptors (particles) on a cartesian coordinate plane by using Matlab. Sources of position uncertainty include the size of the probe and that of the molecule. Since the diameter of the probe is approximately $10 \mathrm{~nm}$, inter-receptor separations smaller than this size can not be resolved and the two receptors are recorded as a single particle. It is important to note that as the experimental techniques are improved, quantifying the transmembrane proteins and understanding the distribution and localization of receptors would be improved. 
The major feature we are interested in is that the particles are distributed unevenly within the images. The detailed fine scale structure of the membrane both in activated and resting cells clustering needs to be observed with a robust and sensitive quantification tools and computations. Therefore, determining the role of spatial organization in the regulation of transmembrane signaling is another key to improve sensitive quantifications.

The predictions from visual analysis and from the Hopkins and Ripley statistic tests are a starting point to characterize the distributions of membrane receptors in both types. There is no overall density variation (we sort of did measure it, the number of points per image is related to the density) trend. The main feature is that particles tend to locate in clusters of sizes ranging from a handful to a few tens. Much of our analysis is focused on this. Then we use hierarchic distance based clustering to identify clusters of particles. The definition of clusters relies on the choice of a characteristic length, which is used to declare that two particles are part of the same cluster. There are subtleties to the method by which the clusters are identified, we will discuss this separately. Overall, we can reliably group the particles into clusters. Either way, one observation is that the number of receptors observed varies significantly between different micrographs. This may be due to uneven labeling, or a non-uniform overall distribution of the receptors.

\subsection{Distributions of Random Geometric Points}

The point distributions derived from our set of TEM micrographs appear random to a large extent, but the majority of the images also exhibit patterns that are non consistent with uniform random placement. We will threfore seek to describe the data with non-uniform random (or probabilistic or stochastic) distributions.

The notion of probability is assigned to the outcomes of a random experiment, that can be repeated any number of times, and has several possible outcomes. The outcome is a random variable (denote it by $X$ ), and the set of all of its possible values is called the sample space $(\Omega)$. For example, the number returned by the rand () function of Matlab can be regraded as a random variable $^{1}$; the sample space $\Omega$ is the interval $[0,1]$. For a subset $A \subset \Omega$ of the sample space, the probability that $X \in A$ (that the outcome of one instance of the experiment is in $A$ ), is the limit, over a large number $N$ of repeats of the experiment, of the ratio of the number $N_{A}$ of times the result is favorable to the total number of trials $(N)$ :

$$
P(A)=P(X \in A)=\lim _{N \rightarrow \infty} \frac{N_{A}}{N}
$$

For example, the probability that rand () returns a number in the interval $A=[0.2,0.3]$ is $P(A)=$ 0.1 . The estimation is correct because the built-in function generates uniformly distributed random

\footnotetext{
${ }^{1}$ Strictly speaking, it is pseudo-random variable.
} 
numbers in $[0,1]$. For this reason, for any interval $[a, b] \subset[0,1]$, the probability is proportional to the length of the interval:

$$
P([a, b])=P(a \leq X \leq b)=b-a .
$$

\subsubsection{Probability Density Function}

We will often compare the observed $(x, y)$ positions of labelled receptors to sets of random variables. Consider a single, real, continuous random variable $X$. It is similar to the outcome of rand (), but its range of possible values is not (necessarily) bounded, and its distribution is not uniform. The behavior of $X$ is characterized by a probability density function (PDF), defined on the set of possible values $\Omega \subset \mathbb{R}, f: \Omega \rightarrow \mathbb{R}_{+}$, which has the following property:

$$
P(X \in[a, b] \subset \Omega)=\int_{a}^{b} f(x) \mathrm{dx}
$$

Any probability density function on $\mathbb{R}$ must satisfy the following conditions

$$
f(x) \geq 0, \quad(\forall x) ; \int_{-\infty}^{\infty} f(x) \mathrm{dx}=1
$$

The probability density function of a continuous, real, random variable $X$ that takes values in $A=[a, b] \subseteq \mathbb{R}$ can be used to obtain the mean value $\langle X\rangle$ of $X$, as well as the expectation $\langle Q\rangle$ of any observable $Q=\varphi(X)$ that depends on $X$,

$$
\langle x\rangle=\int_{a}^{b} x f(x) \mathrm{dx} \quad ; \quad\langle Q\rangle=\langle\varphi(x)\rangle=\int_{a}^{b} \varphi(x) \mathrm{dx}
$$

The notion of probability density can be directly generalized to several (real) dimensions. A random variable in two dimensions can be thought of as a point whose coordinates are given by a pair of random variables $(X, Y)$; the corresponding PDF is defined on $\mathbb{R}^{2}$ and takes positive real values, $f: \mathbb{R}^{2} \rightarrow \mathbb{R}_{+}$. The probability that the two dimensional random variable (random point) takes values in a region $A \subset \mathbb{R}^{2}$, is the integral of the probability density over the region,

$$
P((X, Y) \in A)=\int_{(x, y) \in A} f(x, y) \mathrm{dx} \mathrm{dy} .
$$



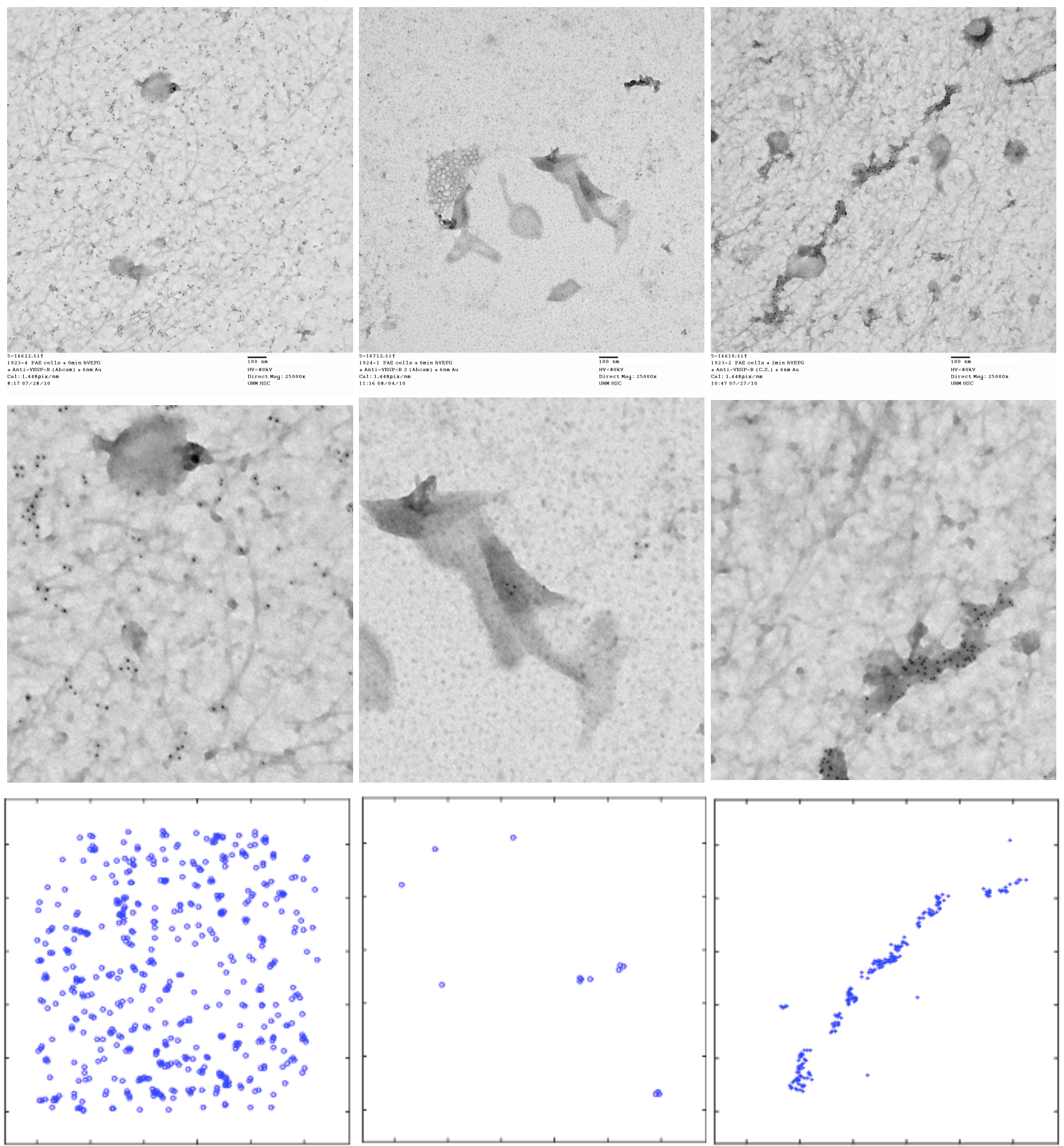

Figure 6: Transmission electron microscopy (TEM) images of nano-gold labelled VEGF receptors on the membrane of PAE-KDR cells and maps of particles. The gold particles appear as dark spots, whose coordinates are extracted in a semi-automatic procedure. Top row: high resolution images; Middle row: details of each image; Bottom row: particle maps generated using the particle coordinates. 


\subsubsection{Cumulative Distribution Function}

The cumulative distribution function (CDF) $F(x)$ describes the probability that a (one dimensional) continuos random variable $X$ takes on a value less than or equal to a number $x$. The CDF is useful in connecting continuous and discrete distributions. The CDF is obtained by integrating a continuous PDF $f(x)$ :

$$
F(x)=P(X \leq x)=\int_{-\infty}^{x} P(\zeta) d(\zeta)
$$

If $X$ has discrete set of possible values $\left\{x_{1}, x_{2}, \cdots, x_{k}\right\}$ with probabilities $p_{1}, p_{2}, \cdots p_{k}$ the CDF is

$$
F(x)=P(X \leq x)=\sum_{X \leq x_{j}} p_{j}
$$

It is useful to clarify what 'uniform distribution' means in terms of probability densities. A prototypical example is the PDF for points uniformly distributed on a line segment of length $L$. Since the points are uniformly distributed, we expect the PDF to be constant, $f(x)=\lambda$, for $x \in[0, L]$, and zero otherwise. The probability that a random point falls within $x$ and $x+\delta x$ is ${ }^{2} \delta P=f(x) \delta x$. Adding up the probabilities for a set of intervals that form a partition of $[0, L]$ should equal 1 ; it also amounts to a Riemann sum that approximates the integral of the PDF:

$$
1=\int_{0}^{L} f(x) d x=\int_{0}^{L} \lambda d x=\lambda L \Rightarrow \lambda=f(x)=\frac{1}{L} .
$$

The mean value or expectation of $X$ is:

$$
\langle X\rangle=\int_{0}^{L} x f(x) \mathrm{dx}=\int_{0}^{L} \frac{x}{L} \mathrm{dx}=\frac{1}{L} \cdot \frac{L^{2}}{2}=\frac{L}{2} .
$$

The CDF may be calculated by integrating from the lower end of the range of values ( 0 in this case):

$$
F(x)=\int_{0}^{x} f(\xi) \mathrm{d} \xi=\int_{0}^{x} \frac{1}{L} \mathrm{~d} \xi=\frac{x}{L} .
$$

In the two dimensional case (see Fig.7 consider a random point uniformly chosen from a circle of radius $R>0$, defined as

$$
\mathcal{C}(R)=\left\{(x, y) \in \mathbb{R}^{2} \mid\left(x^{2}+y^{2}\right)^{1 / 2} \leq R\right\} .
$$

\footnotetext{
${ }^{2}$ We will generally assume that $\delta x$ is small compared to $x,|\delta x / x| \ll 1$.
} 
In this case, the PDF $f(x, y)$ will take a constant value $\lambda>0$ for all $(x, y)$ such that $\left(x^{2}+y^{2}\right)^{1 / 2} \leq$ $R$, and should be zero otherwise. Again, the value of $\lambda$ is obtained from normalization:

$$
1=\int_{-\infty}^{\infty} \mathrm{dx} \int_{-\infty}^{\infty} \mathrm{dy} f(x, y)=\int_{x^{2}+y^{2} \leq R^{2}} \lambda \mathrm{dx} \mathrm{dy}=\lambda \cdot\left(\pi R^{2}\right) \Rightarrow \lambda=\frac{1}{\pi R^{2}}
$$

The probability that the point falls in any subset $A \subseteq \mathcal{C}(R)$ of the circle is the ratio between the area of $A$ and that of the circle:

$$
P((x, y) \in A)=\int_{A} f(x, y) \mathrm{dx} \mathrm{dy}=\frac{1}{\pi R^{2}} \int_{A} 1 \mathrm{dx} \mathrm{dy}=\frac{s(A)}{\pi R^{2}} .
$$

It is useful to consider the probability that the point falls within a circle of radius $r \leq R$; by Eq.1.14, this is simply the ratio of the areas of the two circles, $\left(\pi r^{2}\right) /\left(\pi R^{2}\right)=(r / R)^{2}$. We may regard the distance of the random point $(X, Y)$ from the origin ${ }^{3}, \rho=\sqrt{X^{2}+Y^{2}}$, as a random variable. It is real and continuous, but it can only take non-negative values. The cumulative distribution function $F(r)$ (corresponding to $\rho \leq r)$ is precisely the probability that the random point $(X, Y)$ is located within the circle $\mathcal{C}(r)$. By Eq. 1.7, the PDF $f(r)$ is the derivative of the $\mathrm{CDF}$, and can be calculated as follows:

$$
f(r)=\frac{\mathrm{d}}{\mathrm{dx}} F(r)=\frac{\mathrm{d}}{\mathrm{dx}}\left(\frac{r^{2}}{R^{2}}\right)=\frac{2 r}{R^{2}}
$$

The mean radius works out to

$$
\langle\rho\rangle=\int_{0}^{R} r f(r) \mathrm{dr}=\frac{2}{R^{2}} \cdot \frac{R^{3}}{3}=\frac{2}{3} R .
$$

Before moving on to more detailed calculations, let us clarify how the detailed description of a single random point relates to the distributions of many points we want to analyze. The simplest assumption is that a sample of $N$ points distributed in an area (such as the field of view of a micrograph) may be regarded as $N$ distinct, independent outcomes of the same "random experiment", which consists of generating one random point $(X, Y)$. Therefore, the probability of having $N_{A}$ of the $N$ points in a given area of the image is an approximation of the "true" probability $P(A)$, consistent with Eqs. 1.1 and 1.6.

Consider now that we have $N$ particles, each following the same PDF $f(x, y): \mathbb{R}^{2} \rightarrow \mathbb{R}_{+}$. Focusing on a region $A \subseteq \mathbb{R}^{2}$, the probability that one particle ends up in $A$ is given by an integral

\footnotetext{
${ }^{3}$ Also known as the Euclidean norm of the vector $(X, Y)$.
} 
as in Eq. 1.6. Since each of the $N$ particles falls into $A$ with this same probability, $N \cdot P(A)$ is the average number of particles that should fall into $A$ :

$$
\left\langle N_{A}\right\rangle=N \cdot P(A)=N \cdot \int_{(x, y) \in A} f(x, y) \mathrm{dx} \mathrm{dy}=\int_{(x, y) \in A} N \cdot f(x, y) \mathrm{dx} \mathrm{dy} .
$$

The integrand in the last version of Eq. 1.17 can be interpreted as the averaged local particle density, corresponding to $N$ independent particles that are each distributed randomly, according to the $\operatorname{PDF} f(x, y)$.

\subsubsection{Deriving the Nearest Neighbor Probability Distribution Function}

To obtain the PDF of the nearest neighbor distance, in a set of uniformly distributed random points, we calculate the probability for a point falling between $r$ and $r+d r$ from a randomly chosen point $A{ }^{4}$ As shown in Figure (7), the randomly chosen point is the highlighted (yellow) point in the center of the circle.

The highlighted point ( $M$ in Fig.7) is the nearest neighbor to $A$ only if there are no other random points closer to $A$. We want to calculate the probability for the highlighted green point falling between $r$ and $r+d r$, but nothing inside of $r$. We will use $w(r)$ to denote the corresponding nearest neighbor probability distribution function, and $w(r) d r$ is the probability ${ }^{5}$ that the nearest neighbor of a randomly chosen point $A$ is at a distance between $r$ and $d r$. Eqs. 1.3 and the normalization property Eq. 1.4 provide the probability that the nearest neaighbor distance $R$ is below or above a given positive value $r$,

$$
P(R<r)=\int_{0}^{r} w(\rho) d \rho ; P(R>r)=1-\int_{0}^{r} w(\rho) d \rho .
$$

We assume that there are $\lambda=N / A$ average number of points per unit area distributed randomly. Then $w(r) d r$ is the product of the probability of no points falling closer than $r$ and the probability $\lambda \cdot 2 \pi r d r$ that there is a point between $r$ and $r+d r$ which is,

$$
\begin{gathered}
w(r) d r=\left(1-\int_{0}^{r} w(\rho) d \rho\right) \cdot 2 \pi \lambda r d r \Leftrightarrow \\
w(r)=2 \pi \lambda \cdot r \cdot\left(1-\int_{0}^{r} w(\rho) d \rho\right) .
\end{gathered}
$$

\footnotetext{
${ }^{4}$ Because the distribution is uniform, the result should be the same, independently of the location of $A$ or whether it is part of the sample.

${ }^{5}$ Again, we assume $\mathrm{dr} \ll r$ and therefore $|w(r) \mathrm{dr}| \ll 1$.
} 


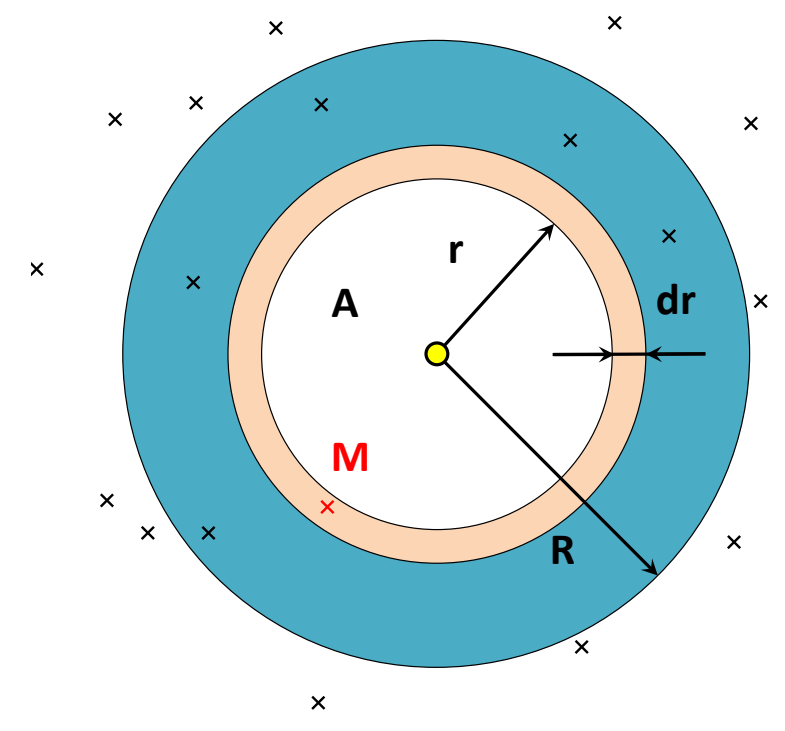

Figure 7: The randomly chosen point is the highlighted yellow point in the center of the circle where we want to calculate the probability for point $M$ (red) falling between $r$ and $r+d r$, but nothing inside of $r$.

By differentiating the equation with respect to $r$ and applying Leibniz' rule to the integral, we obtain

$$
\begin{aligned}
\frac{d w(r)}{d r} & =2 \pi \lambda r\left(-\frac{d}{d r} \int_{0}^{r} w(\rho) d \rho\right)+2 \pi \lambda\left(1-\int_{0}^{r} w(\rho) d \rho\right) \\
& =-2 \pi \lambda r \cdot w(r)+2 \pi \lambda\left(1-\int_{0}^{r} w(\rho) d \rho\right) .
\end{aligned}
$$

Now if we go back to Eq. (1.19), notice we can express the integral term as $w(r) / r$,

$$
w^{\prime}(r)=-2 \pi \lambda r w(r)+\frac{w(r)}{r} \Rightarrow \frac{w^{\prime}(r)}{w(r)}=-2 \pi \lambda r+\frac{1}{r}
$$

Using $\frac{d}{d r} \ln w(r)=\frac{w^{\prime}(r)}{w(r)}$, we obtain

$$
\frac{d}{d r} \ln w(r)=-2 \pi \lambda r+\frac{1}{r} \Rightarrow \ln w(r)=-\pi \lambda r^{2}+\ln r+\mathcal{C}^{\prime} \Rightarrow w(r)=\mathcal{C} r e^{-\pi \lambda r^{2}}
$$

In the second step, we redefined the integration constant $\mathcal{C}=\exp \mathcal{C}^{\prime}$. Its value is set by normalization, and the final result is:

$$
w(r)=2 \pi \lambda r \exp \left(-\pi r^{2} \lambda\right)
$$

The Eq. (1.20) is the nearest neighbor distribution function in two dimensions. Generalizations include the distribution of the second nearest or next-neighbor distance, and similar calculations in $d=1,3, \cdots$ dimensions. 


\subsection{Traditional Measures of Clustering}

One of our goal is to characterize the distribution of receptors in our micrographs, in a way that quantifies the degree of clustering. This is useful as a way to classify such images and to compare how the patterns change between different experimental conditions or cell types. It is also a prerequisite for any mathematical modeling that aims at explaining the observed distributions. This chapter of the dissertation is concerned with describing the point distributions. We are interested in deviations from uniform distribution, and the presence of structure, non-random features, especially clustering. In general, a cluster is a subset of a set of items (points in our case); members of a cluster are in some way closer to each other than to the rest of the elements in the set. Clustering will be discussed at length in section 1.4.

All the statistics we will discuss here are based on the distances between receptors, clusters and random points within and between clusters. A widespread approach to cluster analysis relies on the mutual (Euclidean) distance between pairs of points. The definition of the distance between two particles between $p_{1}=\left(x_{1}, y_{1}\right)$ and $p_{2}=\left(x_{2}, y_{2}\right)$ is as follows:

$$
d\left(p_{1}, p_{2}\right)=\sqrt{\left(x_{1}-x_{2}\right)^{2}+\left(y_{1}-y_{2}\right)^{2}}
$$

We will sometimes refer to the "distance" between a point and a cluster of points. If $C=$ $\left\{p_{i}=\left(x_{i}, y_{i}\right)\right\}$ is a set of points, then the distance between a point $q=(u, v)$ and the cluster $C$ is the smallest distance between the solitary point $q$ and any point in $C$ :

$$
d(q, C)=\min _{p_{i} \in C}\left(q, p_{i}\right)
$$

Given $N_{T}$ random points distributed uniformly in a total area $A_{T}$, the average number of points that fall into a subdivision of it (of area $A_{1}$ ) should be simply proportional to the corresponding area: $N_{1}=A_{1} \cdot \lambda$, where $\lambda=N_{T} / A_{T}$ is the average density of particles. The NND distribution (Eq. 1.20) is one of several statistical measures used to test the uniformity of a distribution of points. If we have a sample of points (locations), the theoretical formula can be compared directly to the observed values of the nearest-neighbor distance. We regard each image as a sample like this. Other statistical measures, such as Ripley's K and L functions, as well as the Hopkins statistic, compare a set of values calculated from the sample to distributions that would correpond to the random placement of points.

\subsubsection{Nearest Neighbor and Next Nearest Neighbor Distance Distributions}

The nearest-neighbor distance (NND) is obtained by selecting, for each point in a distribution, the point from the rest of the set that is the closest. It gives a measure of the local density of points. If 
points are distributed uniformly, then the average nearest-neighbor distance should be close to the radius of the area available to each point. The actual distribution can be derived analytically for a random configuration (see 1.2.3 for the derivation).

$$
w(r)=2 \pi \lambda r \exp \left(-\pi r^{2} \lambda\right)
$$

The above equation gives the nearest neighbor distribution function in two dimensions. Nearest neighbor distance distributions consist of the distance between each each particle and its nearest neighbors location. If the average distance is less than the average for a random distribution, the distribution of the clusters analyzed are considered clustered. If the average distance is greater than a random distribution, the feature is considered dispersed. Since we are interested in the effect of clustering phenomenon on cell signaling, we expect that the major effect of clustering will come from the density of particle points with an important secondary effect coming from the size of the clusters [35]. Therefore, a calculation and an analysis of the nearest-neighbor distances for small distances is important. (See Fig.8 for a sample nearest-neighbor distance distribution plots.)

We also constructed next-nearest neighbor distance distributions for all the images. Similarly to the nearest neighbor distributions (Fig.8) obtained from our set of micrographs, the next neraest neighbor distribution also show consistent deviations from random prediction. A significant fraction of the distribution is well approximated by the theoretical distribution corresponding to a smaller inter-particle distance, with a correction for a minimal seperation. A smaller fraction of the distances is outside this high density mode. The next nearest neighbor distributions (Fig.8) may be more relevant to clustering, since VEGF receptors may for dimers, and thus the nearest neighbor of a receptor may be its dimer partner. The majority of the distance distribution falls in a high density mode that is well approxmated by the dashed curve.

\subsubsection{The Hopkins Statistic Test}

Consider a data set $D$ consisting of $N$ particle (or probe) coordinates $\left\{r_{i}=\left(x_{i}, y_{i}\right)\right\}_{i=1 \ldots N}$. The Hopkins statistic is defined as follows. For $m \ll N$, choose $m$ random sampling points $s_{i}=$ $\left(x_{i}, y_{i}\right)$ in the area covered by the image. Let $\tilde{r}_{k}=r_{j_{k}}$ be the positions for $m$ randomly chosen probes from $D$. We define

$$
U=\sum_{i=1}^{m} d^{2}\left(s_{i}, D\right), \quad W=\sum_{k=1}^{m} d^{2}\left(\tilde{r}_{k}, D\right)
$$

where the point to cluster distances $d\left(s_{i}, D\right)$ and $d\left(\tilde{r}_{k}, D\right)$ are defined as in Eq.1.22. The Hopkins statistic is

$$
H=\frac{U}{U+W}
$$



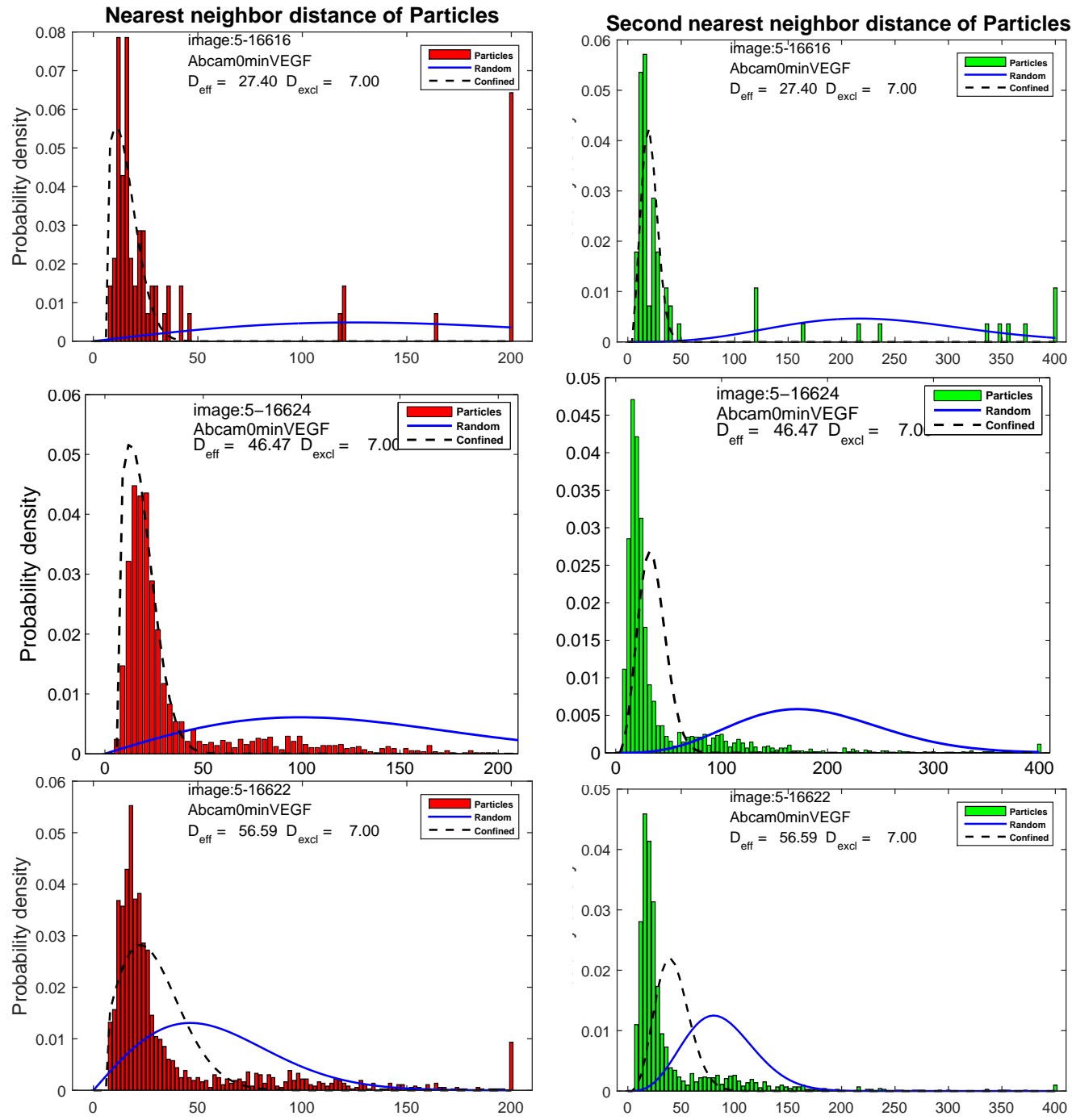

Figure 8: Analysis of clustering in the resting cells using nearest and next nearest neighbor distance distributions. Compared to plots on the left, a larger fraction of the distances on the right falls outside the high density mode, possibly forming a second mode that corresponds to inter-cluster distances. 
The $H$ values are in the interval $[0,1]$. The expected value for uniformly distributed data is 0.5 . $H>0.5$ indicates clustering in the image. The plots in Fig.9 are histograms obtained by repeated samplings of $H$ for different random selections of points. The Hopkins statistic does not provide the number of clusters. In our study, we estimate the statistic for the first nearest neighbor distance. Hopkins statistics test which indicates some clustering within the randomly generated data as the bar graph has moved to the right of the expected curve for random data. The reason is that the Hopkins test is not accurate for data sets that contain a small numbers of points. It is clear that a more quantitative assessment would really be helpful in quantifying the clustering in this data.

\subsubsection{The Ripley Statistic Test}

\section{Ripley's K function}

The $K$ function is

$$
K(r)=\lambda^{-1} A(r)
$$

where $A(r)$ is the area of a disk with a radius $r$ of a randomly chosen particle, and $\lambda$ is the density (number per unit area) of particles. Or alternative definition is mean of nearest-neighbor distance from random points to their nearest neighbors. Because there are $n$ particles in $D$, the mean of particles in a disk of radius $r$ about each of the particles is

$$
A(r)=\lambda \pi r^{2}
$$

for a uniformly distributed data. The Ripley's $K$ functions is

$$
K(r)=\frac{A(r)}{\lambda}
$$

with the expected value $\pi r^{2}$ of the uniformly distributed particles.

\section{Ripley's L Function}

The $L$ function is

$$
L(r)=\sqrt{\frac{K(r)}{\pi}}=\sqrt{\frac{A(r)}{\lambda \pi}}
$$



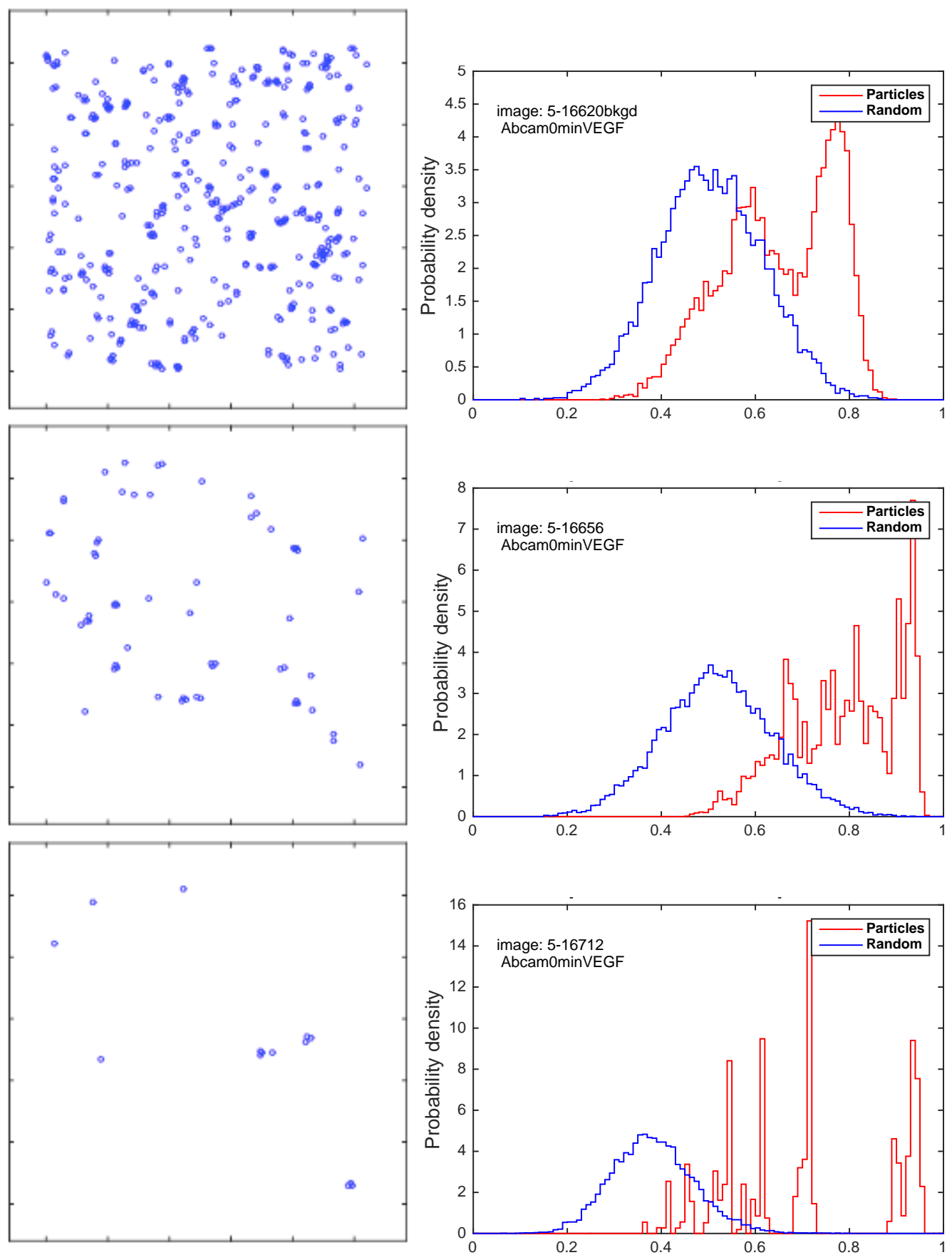

Figure 9: Analysis of clustering in the resting cells using the Hopkins statistic. From top to bottom: Experimental samples demonstrating the distribution of VEGFR on membrane sheets. 

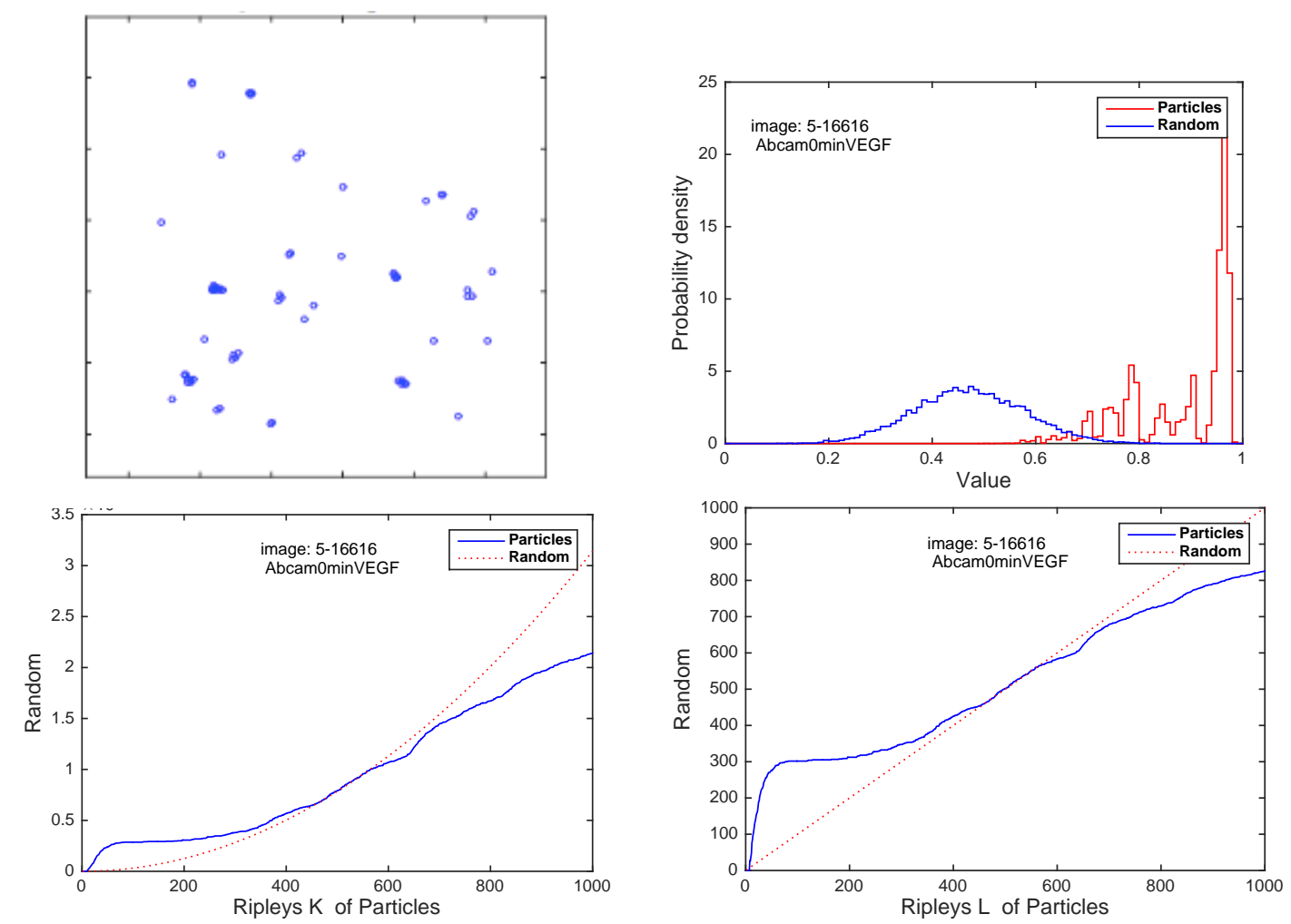

Figure 10: Hopkins and Ripley K and L statistic tests for image:5-16616 given by a plot of the statistic for simulated random data to be compared with a plot of the statistic computed from the experimental data and cluster centroids.

which is a preferred usage for Ripley statistic test in recent publications [22, 23]

Ripley statistics in Figure (10) are given by a plot of the statistic for simulated random data to be compared with a plot of the statistic computed from the experimental data.These methods can distinguish between more and less clustered data. However, they do not provide a straightforward quantitative measure of the extent of clustering. Both are sensitive enough to test our hypothesis, and each provides different information about the clustering.

The plots of the statistics for simulated random data are compared with the plot of the statistics computed from the experimental data [35]. These methods are applied to distinguish between more and less inter-clustered data and intra-clustered data. 

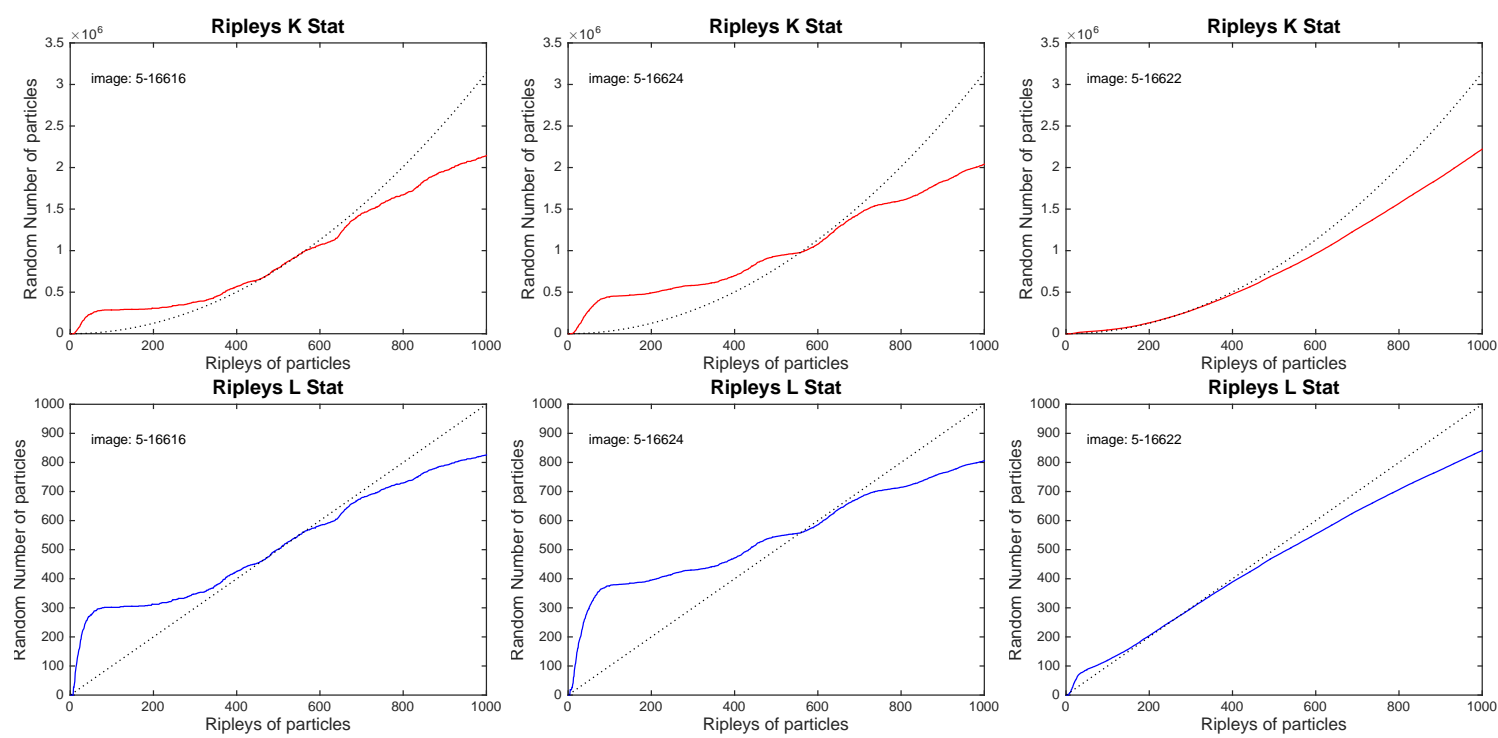

Figure 11: Ripley's K and L for a few images. The image on the right is not typical.
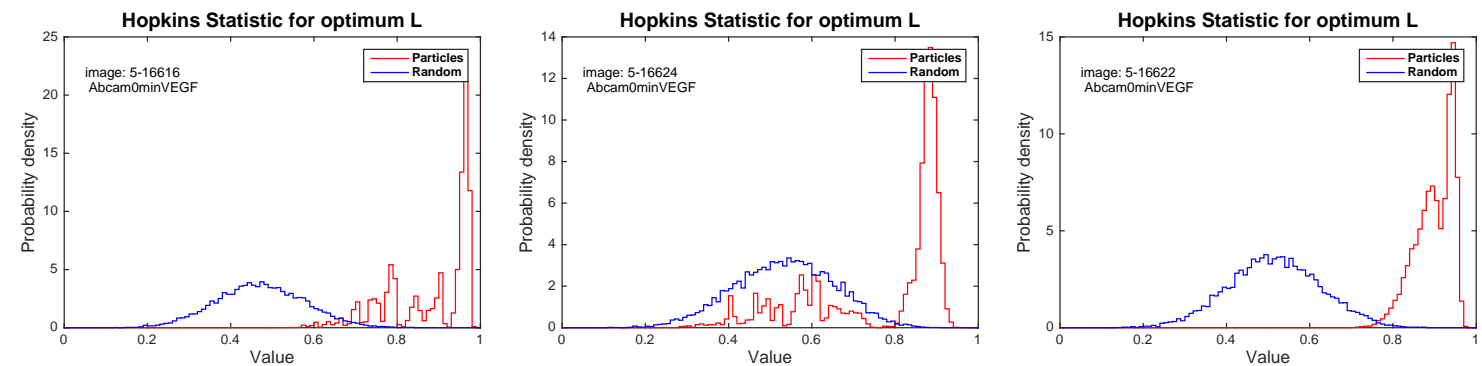

Figure 12: Hopkins test results for a the same images as shown in Fig.11. The image on the right is not typical.

\subsubsection{Results}

The analyses outlined above were performed for each of the images in the dataset. This was made possible by automating the process, and performing the calculations in a script that loops over the images. We generated several plots for each image file, for each type of analysis. It is not possible or useful to include all of these results here. Below I present brief summaries of the findings and illustrate with samples of individual results or with summary representations.

The Hopkins and Ripley tests are traditionally used to establish whether a set of geometric points is clustered. With very few exceptions, Ripley tests confirmed that indeed, the points in our images exhibit clustering, and their distribution is not consistent with random uniform placement. Sample plots are shown in Fig. 12. 


\begin{tabular}{|l||l|l|l|}
\hline \multicolumn{4}{|c|}{ Experimental Data Sets } \\
\hline $\begin{array}{l}\text { Antibody Type } \\
\text { and Time }\end{array}$ & $\begin{array}{l}\text { Total Number of } \\
\text { Images }\end{array}$ & $\begin{array}{l}\text { Total Number of } \\
\text { Receptors }\end{array}$ & $\begin{array}{l}\text { Average Number } \\
\text { of Receptors }\end{array}$ \\
\hline Abcam 0 min & 21 & 2625 & 125 \\
Abcam 2 min & 14 & 2457 & 175 \\
Abcam 5 min & 29 & 3930 & 135.5 \\
CS 0 min & 8 & 1015 & 126.8 \\
CS 2 min & 10 & 935 & 93.5 \\
\hline
\end{tabular}

Table 2: Experimental data sets: column 1 is the type of antibody with related time $t$ in minutes at which the cells were fixed, columns 2 through 4 gives the number of images in total, total number of particles and the average number of particles per image for corresponding data set respectively.

As it is shown in 2, we have 81 micrographs that consist of two different subsets; Abcam and CS. We describe the general behavior of data by using histograms after we apply a set of analyzing methods. Those analyzing methods include deriving clustering length scale empirically, then based on the length scale we apply the DRA to find the final domains which refers to clusters for our static data. Then we plot histograms for all those corresponding described parameters to seek the distribution of data sets. The bars represent the frequency of occurrence by classes of data. Our histograms as shown in Fig. 13 show the basic information about the data set, such as central location, width of spread, and clusters. We use histograms to assess the current situation of the micrographs and to study results of improvement actions before we apply our predictive model which will be described in the next chapter. The histograms shape and statistical information help us decide how to improve our modeling approach. If the system is stable, we can find unknown predictive parameters about the future system of data. After improvement action has been carried out, we continue collecting data and making histograms to see if our predictive model has worked. Our histograms for different parameters and features of the data have shown consistent deviations which we think that outliers for a handful set of data usually remain the same, and the majority of the data follow a normal distribution in the first instant.

\subsection{Hierarchic Clustering}

Clustering is the task of grouping a set of objects in such a way that more similar objects are in the same group which is called a cluster. The clusters are more similar to each other where as different clusters are less similar. However, we use distance in geometry to group objects in a cluster that are closer to each other. The method of distance based hierarchic clustering has been applied in various contexts [9]. Hierarchical clustering is also known as connectivity based clustering is based on the particles being more related with nearby particles than to the particles that are far 

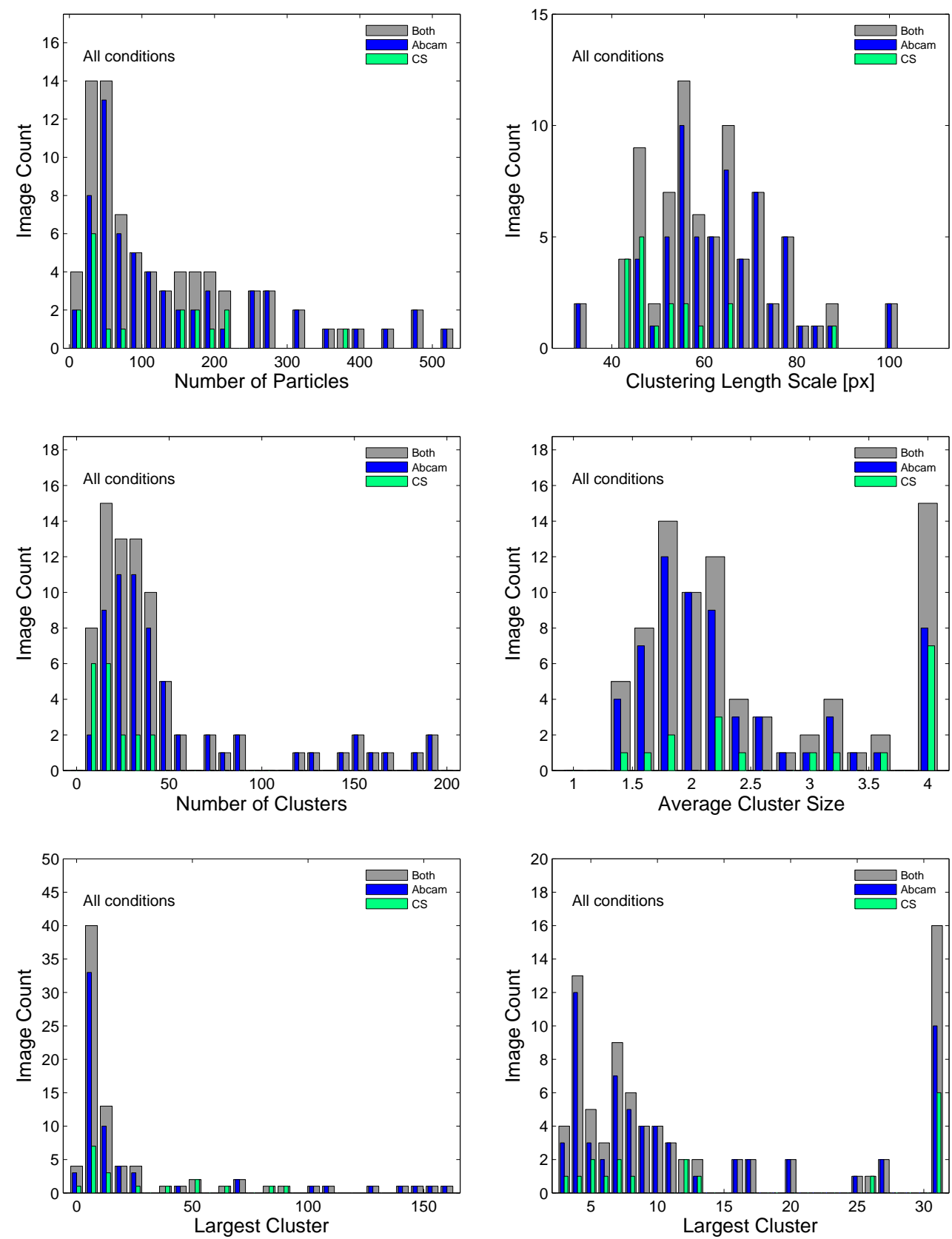

Figure 13: Histograms of all the characteristic information of the data and clusters in each data set after we performed the clustering analysis for each data set. In order to identify the characteristic length between two points that defines a clustering, we use the hierarchic clustering method in two dimensional space. 

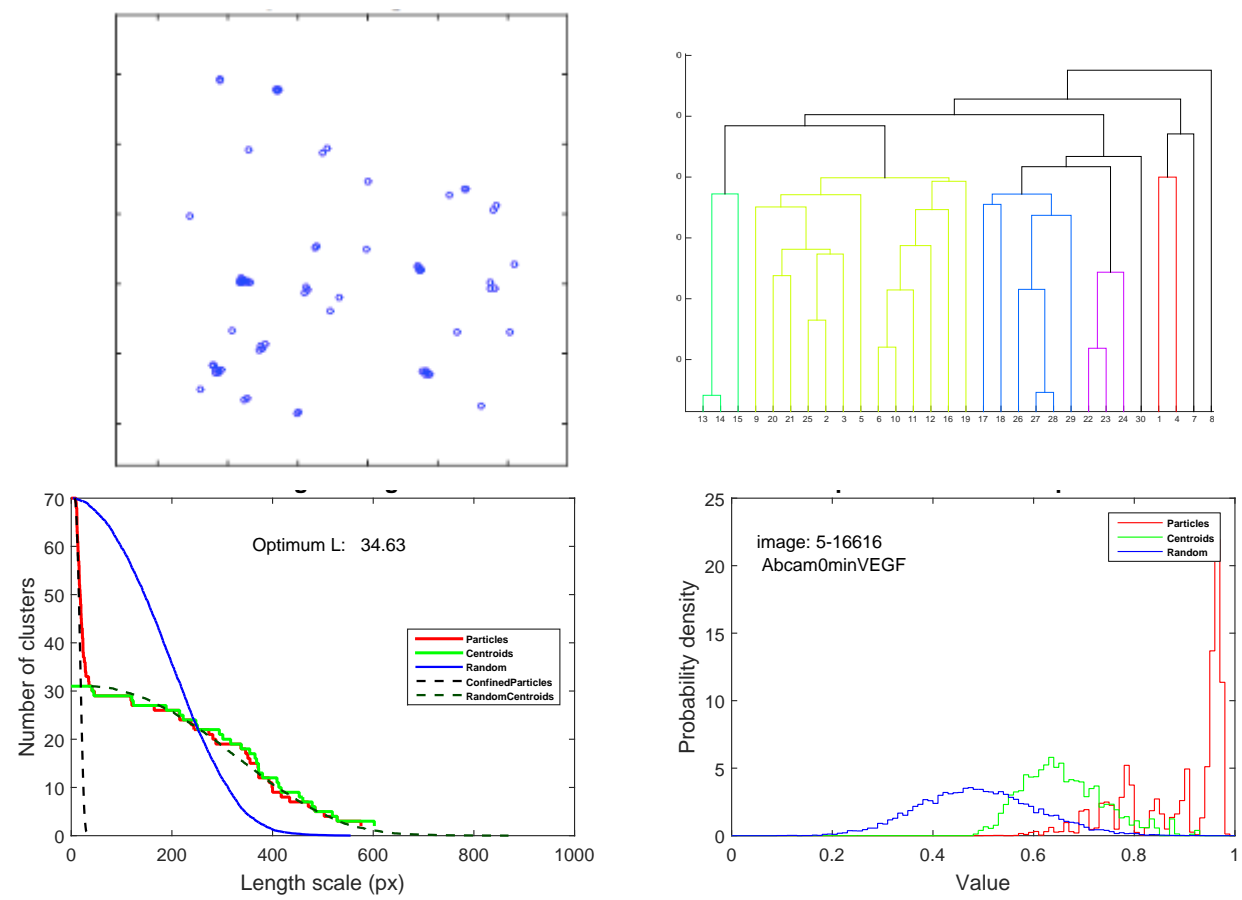

Figure 14: a) particles map of image 5-16616 with 70 particles; b) dendrogram plot of 70 particles with 29 leaf nodes by using linkage function in Matlab; c) cluster scaling curve where the optimal clustering length is $L=34.63$; d) the Hopkins clustering test.

away. With different distances different cluster may form and dendrogram is used to represent those clusters. We use single-linkage clustering where we start with single elements and aggregate them into clusters as in Figure (14). The dendrogram function from Matlab is used to generate and display the hierarchical clustering of the data. Our starting point is the work of Espinoza and coworkers [5], who initially developed the method to analyze the nano-gold labelled membrane proteins.

Given a set of points $\mathcal{P}=\{\mathrm{A}, \mathrm{B}, \cdots\}$ in a metric space of distance $d($,$) , we may group the$ points into distance based clusters defined by a chosen scale $L \geq 0$ as follows.

i. Two points $(A, B)$ are in the same cluster if their distance is less than $L$,

$$
d(\mathrm{~A}, \mathrm{~B}) \leq L \Rightarrow \mathrm{A} \sim_{\mathrm{L}} \mathrm{B} .
$$

ii. The relation $A \sim_{L} B$ is extended by transitivity, i.e., two points are in the same cluster if there is a third point which is in the same cluster with each of them:

$$
\left(A \sim_{L} B \& A \sim_{L} C\right) \Rightarrow B \sim_{L} C .
$$


The relation $A \sim_{L} B$ defined above is also symmetric, and is therefore an equivalence relation on the set of points $\mathcal{P}$. Clusters are uniquely defined as the equivalence classes induced by $\sim_{\mathrm{L}}$.

\subsubsection{Dependence on Length}

The scale $L$ is crucial to the definition of the clusters. If we compare the clusters induced by two different scales $L_{1}<L_{2}$, on the same set of points, we easily find that two points that are in the same cluster with respect to the shorter scale are always in the same cluster with respect to the longer scale,

$$
\left(L_{1} \leq L_{2}\right) \Rightarrow\left((\forall) \mathrm{A}, \mathrm{B} \in \mathcal{P}, \mathrm{A} \sim_{\mathrm{L}_{1}} \mathrm{~B} \Rightarrow \mathrm{A} \sim_{\mathrm{L}_{2}} \mathrm{~B}\right) .
$$

In other words, each cluster induced by $L_{1}$ is a subset of a cluster induced by $L_{2}$, and as the length parameter is increased, the clusters grow in size and their number decreases.

One way to characterize the distribution of particles is to analyze the dependence of the number of clusters on the lengh parameter, $N_{C}(L)$. We have established that $N_{C}(L)$ is a decreasing function for any given set of points. As $L \rightarrow 0$, each point in $\mathcal{P}$ is its own cluster, and as $L$ increases beyond the largest point to point distance, the entire set $\mathcal{P}$ forms a single cluster. ${ }^{6}$

We found through numerical simulations that the curve for randomly distributed points in an area can be obtained as a scaled version of a universal function, as shown in Figure (15). The scaling law is as follows:

$$
N_{\mathrm{C}}(L)=N_{\text {total }} \cdot \phi\left(\frac{L}{\sqrt{A_{\text {total }} / N_{\text {total }}}}\right) .
$$

The knowledge of this universal function is useful in comparing our experimentally derived distributions with corresponding random sets of points.

\subsubsection{Identifying the Optimum Length}

The short distance part of nearest neighbor distributions and cluster scaling curves are consistent with particles distributed randomly and uniformly in a smaller area as it is shown in Fig.16. If

\footnotetext{
${ }^{6}$ The analysis presented here differs from that in [5], which focused on clusters of two or more particles. The curve obtained there would approach 0 , respectively 1 at small and large values of $L$, with at least one maximum in between.
} 


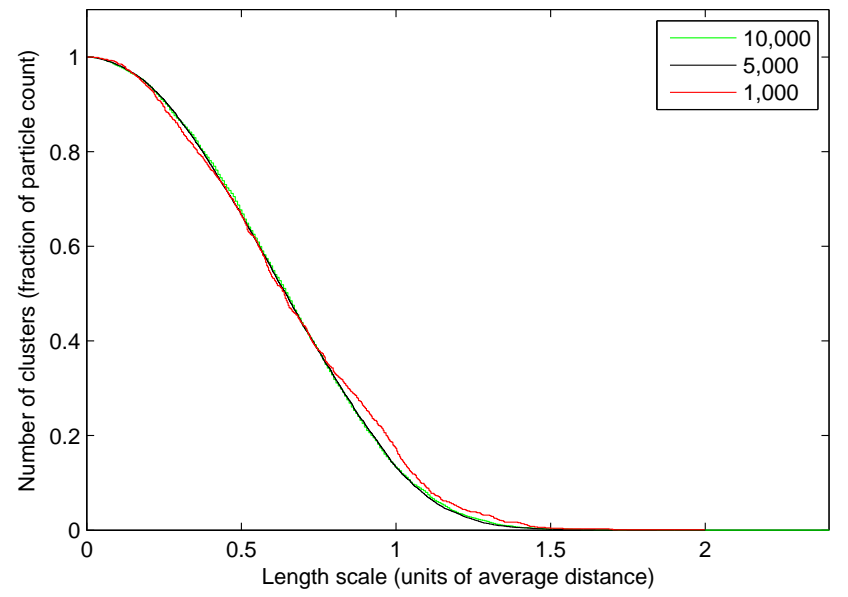

Figure 15: The dependence of the number of clusters $N_{\mathrm{C}}$ on the length scale $L$ (for $N_{\text {total }}$ points distributed randomly in a square of area $A_{\text {total }}$ ) appears to follow a universal function as shown in Eq. (1.31). The curves shown are from $N_{\text {total }}=\{1000,5000,10000\}$ points randomly placed in a unit square.

there was a perfect scale seperation between the distribution of clusters and that of particles within clusters, the $N_{C}(L)$ curve would have plateau. The spatial distribution of particle within clusters is consistent with random placement. We identify the optimal $L$ empirically as two times the $L$ value corresponding to the inflection point.

As a starting point, we visualize the distribution of clusters for various length scales. If the particles are grouped into naturally defined clusters (in the sense of a clear scale separation between intra- and inter-cluster distances), this natural length scale can be interpreted either as the typical, or the largest intra-cluster distance between a particle and its nearest neighbor. The second definition seems more practical since this would result in correctly separating the particles into clusters as it is shown in Fig. 16. The short distance part of nearest neighbor distributions and cluster scaling curves are consistent with particles distributed randomly and uniformly in a smaller area. The definition of cluster which includes number of clusters and cluster sizes of the data with the distribution of particles within clusters varies by choosing different length scales. When the length scale is 30 pixels each particle on the cell membrane behaves as a different cluster, whereas after length scale is 200 pixels the clustering formation merges to be one big cluster as in Figure (18). Therefore, we decided to construct a more reliable mathematical approach to understand what length scale represents each experimental data. We perform hierarchic distance based clustering using the built-in Matlab function linkage(); the output of this function can then be represented as a dendrogram via another function (dendrogram ()$). Z$ is an $(m-1) \times 3$ matrix, where $m$ is the number of particles in an experimental data. Columns 1 and 2 of $Z$ build from cluster indices linked in pairs to form a binary tree. The nodes vary from 1 to number of particles. Nodes are the trivial clusters from which all higher cluster sizes are built. Each newly-generated cluster, corresponding to row $Z(I,:)$, is assigned the index $m+I$ where $m$ is the number of particles in each experimental data 

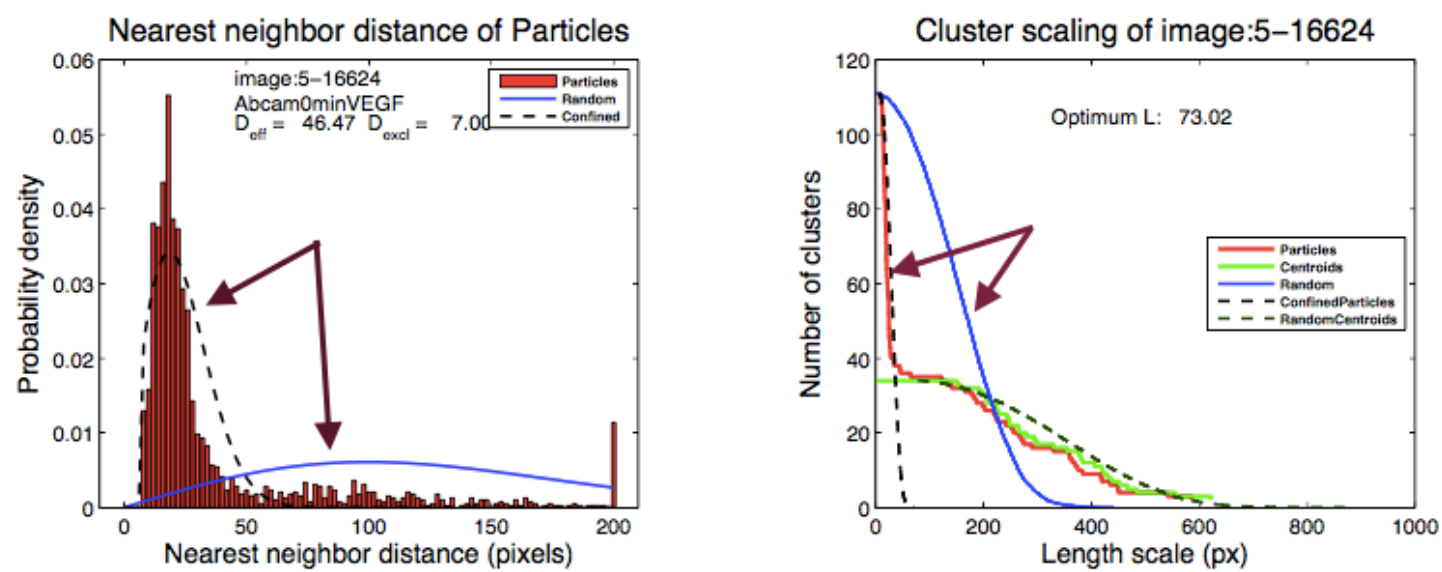

Figure 16: The short distance part of nearest neighbor distributions and cluster scaling curves are consistent with particles distributed randomly and uniformly in a smaller area.
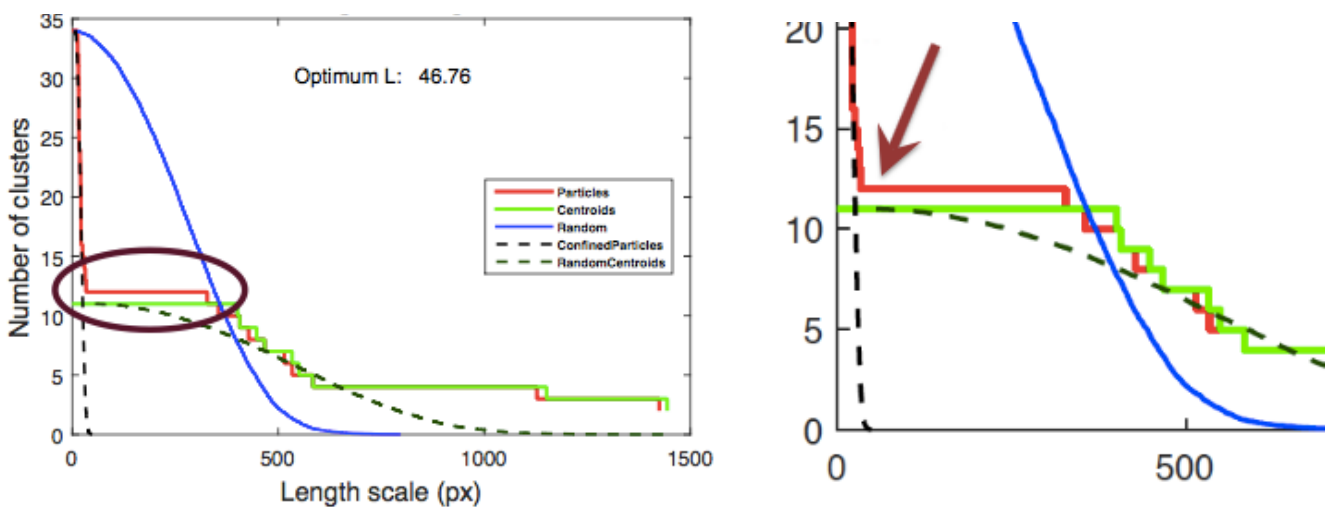

Figure 17: We identify the optimum $L$ of each micrograph empirically as two times the $L$ value corresponding to the inflection point where the plateau starts i.e. in the cluster scaling curve of particles are represented with red curve that shows a dramatic decrease until optimum length scale $L$. 
set. The indices of the two component clusters is contained in $Z(I, 1: 2)$ to form cluster number $m+I$. The interior nodes of the clustering tree includes $\mathrm{m}-1$ clusters. By merging the two clusters in row $Z(I,:)$, the linkage distances are contained in $Z(I, 3)$.

Example: Suppose there are 30 initial nodes and at step 17 cluster 8 and cluster 11 are combined. Suppose their distance at that time is 2 . Then $Z(17,:)$ will be $[8,11,2]$. The formed cluster will then have an index as $17+30=47$. If cluster 42 appears in a later row, it means the cluster created at step 17 is involved into some larger cluster.

The third column of $\mathrm{Z}$ matrix has the information of the distances between two clusters. Therefore, we can now generate the curve $N_{C}(L)$ based on all the distances between two clusters, which we call it cluster scaling curve. The concept of the characteristic clustering distance that will describe the nanoscale distance between particles that are in clusters. The function $N_{C}(L)$ is noisy, as is indicated in (17) for random data and for the experimental data. We plot this curve by length scale versus number of clusters for each experimental data set in two dimensional space. We then investigate the rate of change with respect to the length scale where we think that the twice of maximum slope represents the characteristic length for a data.

\subsubsection{Domain Reconstruction Algorithm and Cluster Analysis}

We have created an algorithm that constructs the size and shape of domains in our model to identify the area and perimeter of the clustering. First we apply the hierarchical clustering with a distance $L$ for a TEM image. The particles in a cluster form a connected graph. The connection graph is built by putting an edge between pair of particles where the distance between them is less than $L$. To extend the contiguous area we add rectangles of height $L$ along convex shape of connection graph. As the process goes on, it circles around the points in a counter-clockwise direction where all the interior points are to the left hand side. When the contour is built, the last point in the contour is identical to the first one as shown in Figure (21). To straighten the boundary of the region defined this way, we extend the area by adding rectangles of height $L$ along the contour of the connection graph in Figure (21(c)). The reconstructed region is the reunion of the inside of the contour graph, and the circles and rectangles around the vertices and edges of the contour (shaded, respectively grey areas on Figure (21(d)). The contour graph or tight contour for a cluster of points is defined by the sequence of boundary points and the segments that connect them. The list is constructed by adding new points to the contour, based on the existing points and a reference direction. As the contour is built, it circles around the points in a counter-clockwise direction, so that all the interior points are to the left hand side. When the process is finished, the last point in the contour is identical to the first one. 

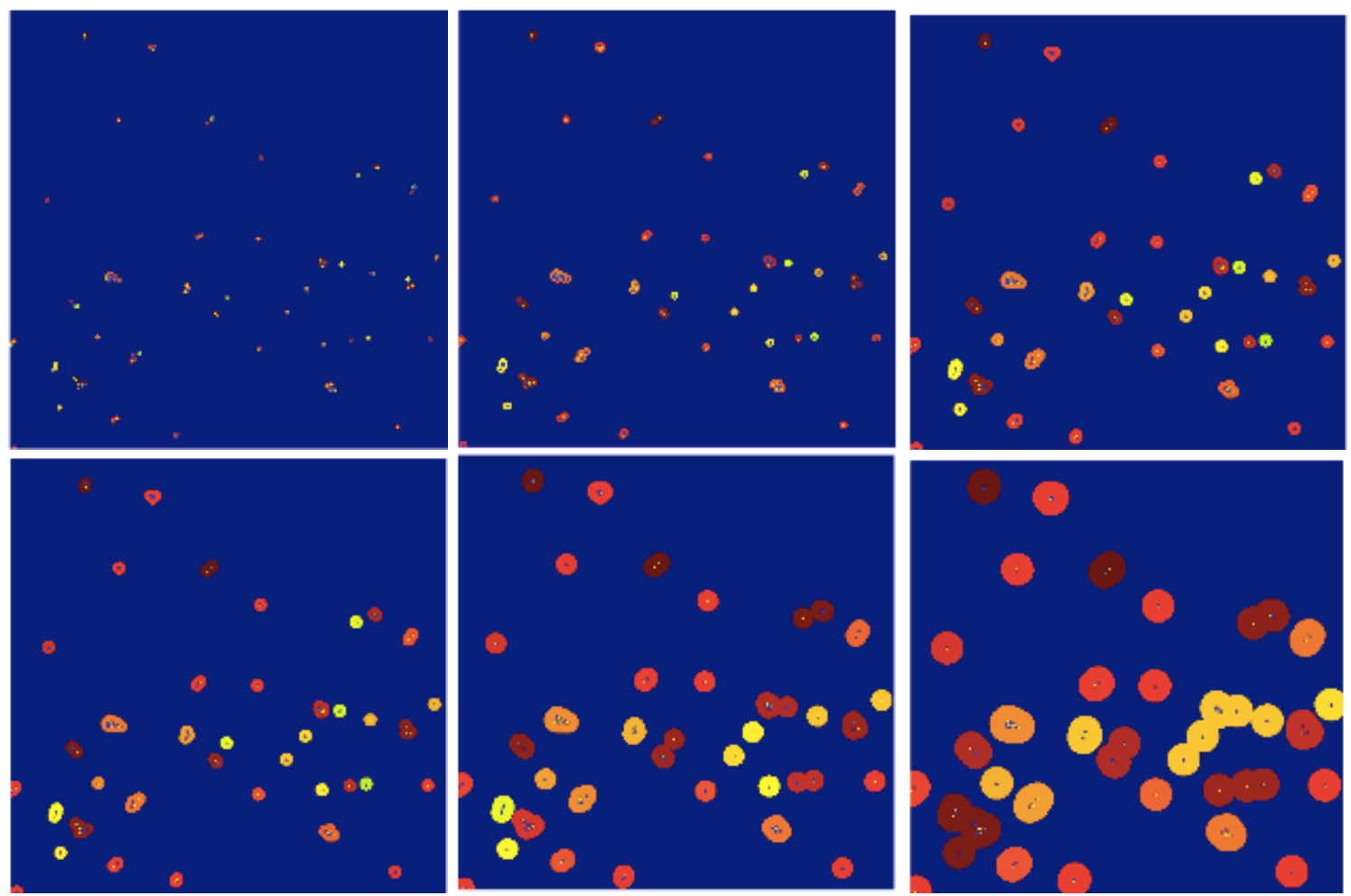

Figure 18: Cluster sizes distribution of image 5-16616 for different length scale $L=$ $\{30,50,70,100,130,200\}$ from top to bottom. Greater length scales lead to have less clusters. 

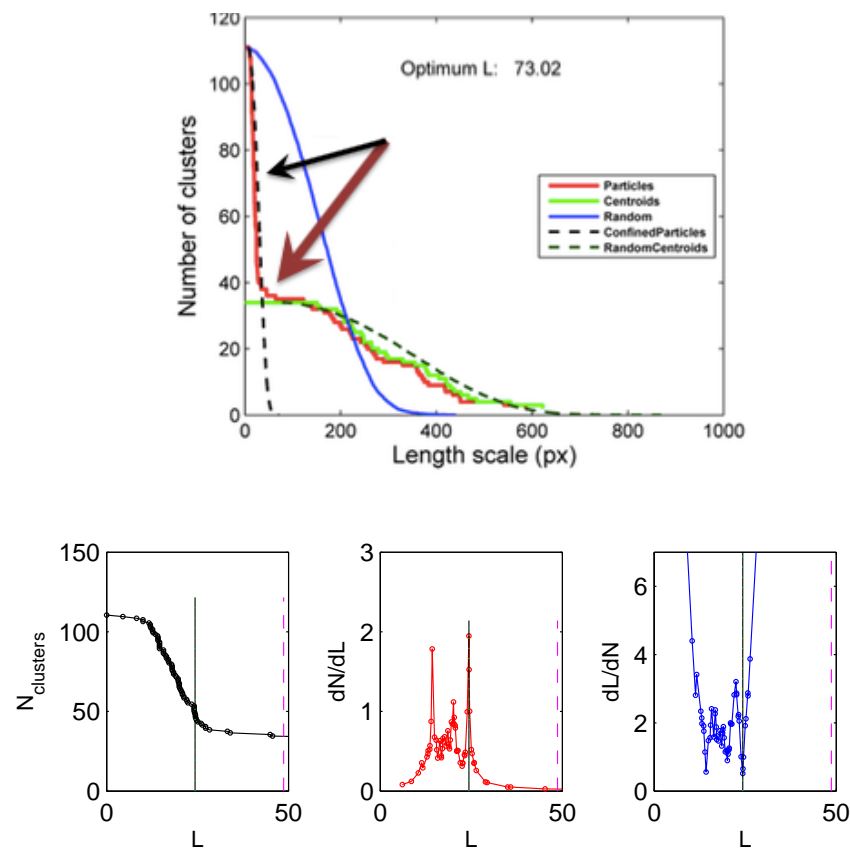

Figure 19: The figure on top is the cluster scaling curve of an image with optimum $L$ around 73 pixels. The red curve represents the particle distribution of image whereas the black dashed curve represents random particles distribution into a smaller area. We take the derivative of this red curve to identify the inflection point. At bottom, the second picture exhibits the identification of the the clustering distance $L$ as the maximum. We then decided to take twice of the maximum of the cerivative curve for all the images empirically.

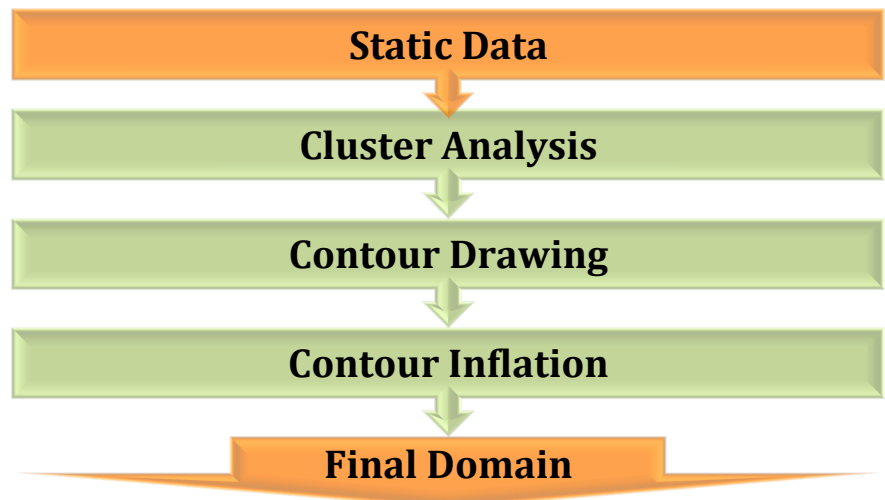

Figure 20: The diagram of how micrographs (static data) are converted to static spatial data and used to determine domain size and distribution. This process goes as reconstructing the domains via a clustering algorithm, contour drawing, and contour inflation. 


\section{Contour Building Algorithm}

1. Start with the rightmost point of the set; begin with the reference direction pointing to the right. (Any particle on the convex hull can be used; the reference direction needs to be pointing toward the outside of the convex hull.)

2. Add new geometric points (particles):

a. Identify all the candidate geometric points in a circle of radius L centered on the last point added (the current point).

b. Draw line segments from the current point to each candidate point. If this intersects a segment in the already identified part of the contour, respective candidate point can be discarded.

c. Order the remaining candidates by the clockwise angle from the reference direction to the segment connecting the current point to the candidate; choose the candidate with the smallest angle and add it to the list.

d. Set reference direction to point from the newly added point back to the previously added point

3. The process terminates when the same segment is added to the contour. The same point may be visited twice, in opposite directions. Upon successful termination, the last point in the list is the same as the first one.

NOTE: The contour defined this way is not unique, but the algorithm always returns a contour that is a refinement of the convex hull of the points, is not self-intersecting, and contains no edges longer than $L$. 

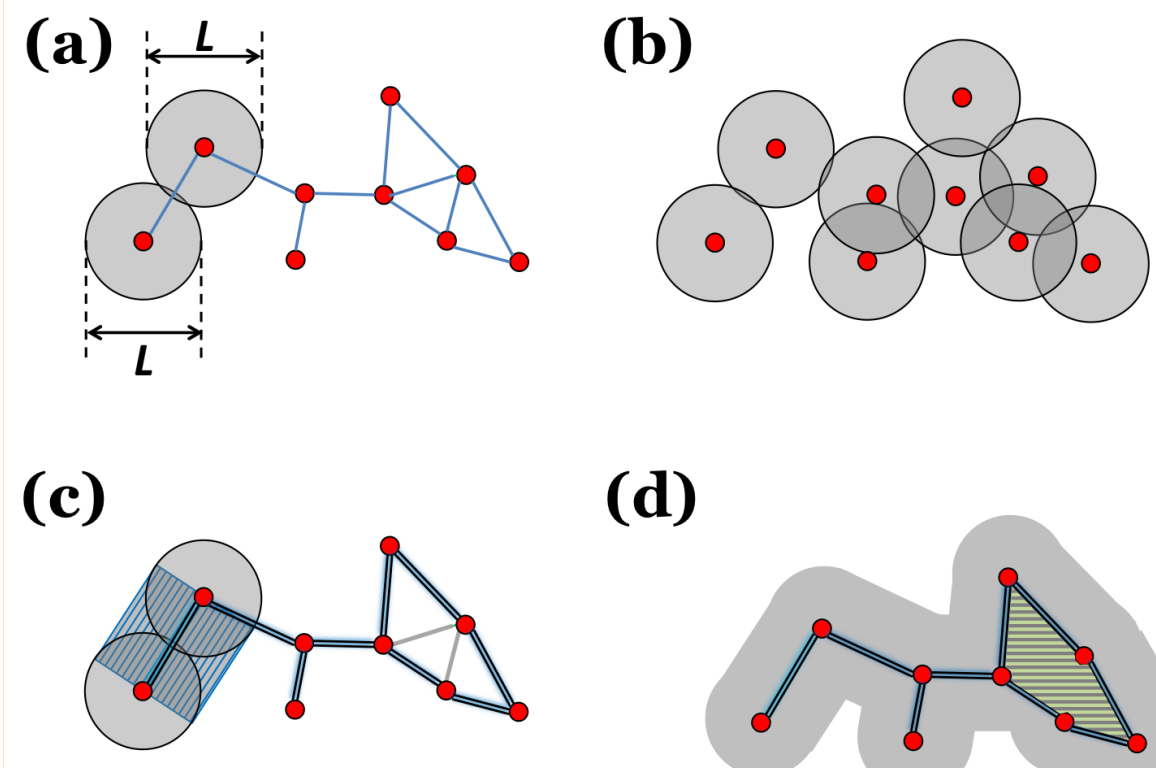

(d)

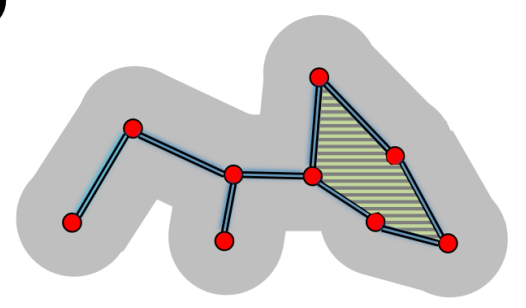

Figure 21: (a) The points in a cluster form a connected graph, where edges connect points whose distance is less than the length scale L. Circles of diameter L centered on two points intersect if and only if the points are connected in the sense described above. (b) We want to define the footprint based on the reunion of all the circles of diameter $L$, centered on the points in the cluster. (c) We first identify the outer contour of the cluster graph (double blue shaded lines). We pad the area defined by the circles by adding rectangles along the edges of the contour graph. (d) The reconstructed region is the reunion of the inside of the contour graph (if any), and the circles and padding rectangles around the vertices and edges of the contour graph [18] 


\section{Chapter 2}

\section{Model}

In this chapter, we use the very first results of descriptive statistics in Chapter 2 in order to quantify the clustering of the biological static data. The main and important thing is that we come up with a model which predicts the size of clusters. Our model explains the micro-domains on the cell surface quantitatively which biologists think that these micro-domains are attractive for receptors. Pre-existing micro-domain model is the best hypothesis that explains the specific compartments that are attractive to the receptors which affects cell signaling. The new statistics based on hierarchical clustering and dendrogram analysis produce numerical values that increase by applying our model. Consequently, it is now easy to check rigorously that our domain reconstruction algorithm produce consistent results to construct the clusters and applying these clusters to our model.

There is no clear understanding of the mechanism of receptor accumulation. This occurs for VEGFR and also other receptor types, for which there is no evidence of a collective binding mechanism $^{1}$. A widely accepted explanation is that receptors accumulate in micro-domains: small, physically delimited areas of the cell membrane that result from partitioning by actin filaments (elements of the cytoskeleton) or are formed by local aggregations of specific types of lipids and / or membrane proteins.

\footnotetext{
${ }^{1}$ Some receptor types, such as FceRI, may form multi-molecular aggregates through cross-linking, which would explain clustering. However, clustering is observed for receptor types for which multiple cross-linking does not occur; this includes EGF receptors as well as FceRI under different circumstances.
} 


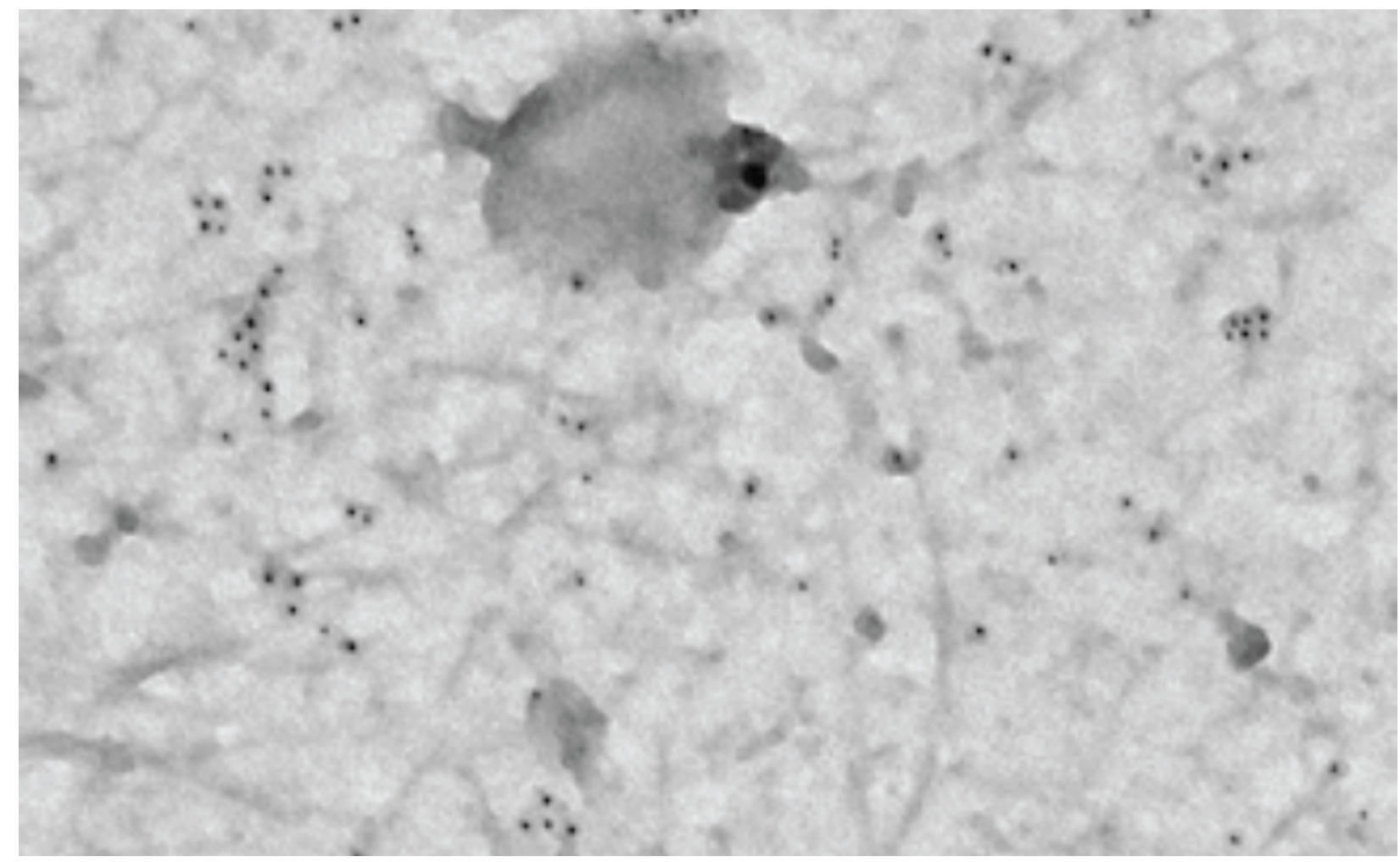

Figure 22: The clusters of labelled receptors appear to be located in regions of the membrane that have different local properties. The interpretation may be somewhat misleading in that the shades on a transmission electron micrograph do not indicate depth, but rather, the ability to absorb electrons. However, the clusters seen in the image are occupy small areas of a different apparent consistency; there are very similar domains that do not contain labeled receptors.

\subsection{Attractive Micro-domains}

The reasons for receptor clustering are not completely understood, but it is generally accepted that it is a consequence of the physical features of the cell membrane, which influence the movement of receptors. Here we rely on a simple hypothesis, that the clusters form in specific regions (small domains) on the cell membrane, due to special physical properties of those regions.

Figure 22 illustrates the idea. The membrane has many of these darker shaded regions (or domain), which seem to be preferred by the receptors. Some of the domains are empty, but the domains altogether represent a small fraction of the total area.

Direct detection of membrane micro-domains is challenging. Available methods provide a range of temporal, spatial and chemical resolutions. Thus, different pictures can arise from different experimental modalities for the cell / receptor combination, and it may not be possible 

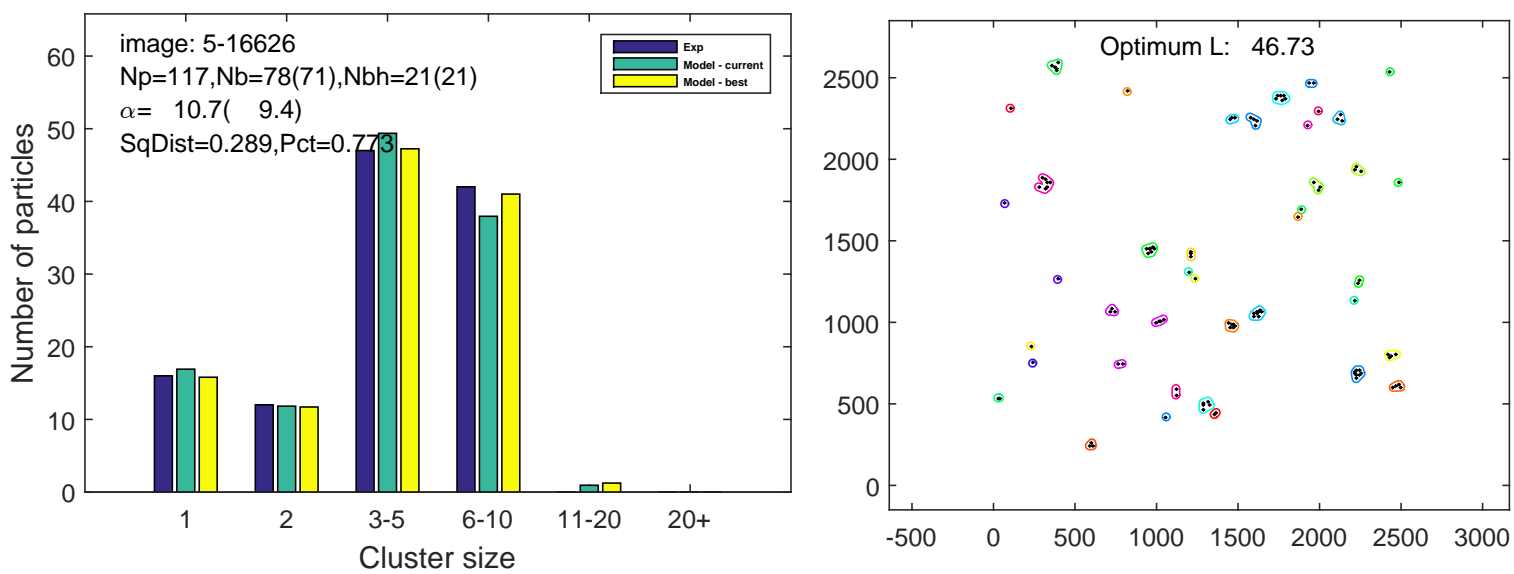

Figure 23: The left panel shows the distribution of particles by cluster size in a somewhat typical micrograph. The distance based cluster identification is shown in the right panel. The simultaneous abundance of moderately large clusters (size 6-10) as well as singleton particles is not consistent with random placement of all particles in a small number of attractive domains.

simultaneously detect several different features of membrane domain properties [4].

Static images of receptors (even in the absence of ligand) typically reveal a clustering pattern, where receptors tend to accumulate in groups ranging from a few to a few tens of receptors. However, clustering is observed for VEGFR and also for other receptor types, for which there is no evidence of a binding mechanism leading to aggregates that contain more than two receptors. Our working hypothesis is that some of the micro- domains found on the cell membrane have a specific molecular composition that results in an affinity for the receptors; receptors may diffuse in and out of the domain boundaries, but the crossing probability is asymmetric.

\subsubsection{A Working Hypothesis for the Mechanism of Receptor Clustering}

Our modeling approach relies on the following hypothesis. The observed clusters result from small domains (micro-domains), whose physical properties cause the freely diffusing receptors to locate preferentially in them. We assume that the observed clusters are typically receptors located in one such domain. However, depending on the number of receptors and the density and size of the micro-domains, some observed clusters may be groups of receptors that are not in a micro-domain, but are located close to each other by chance. 


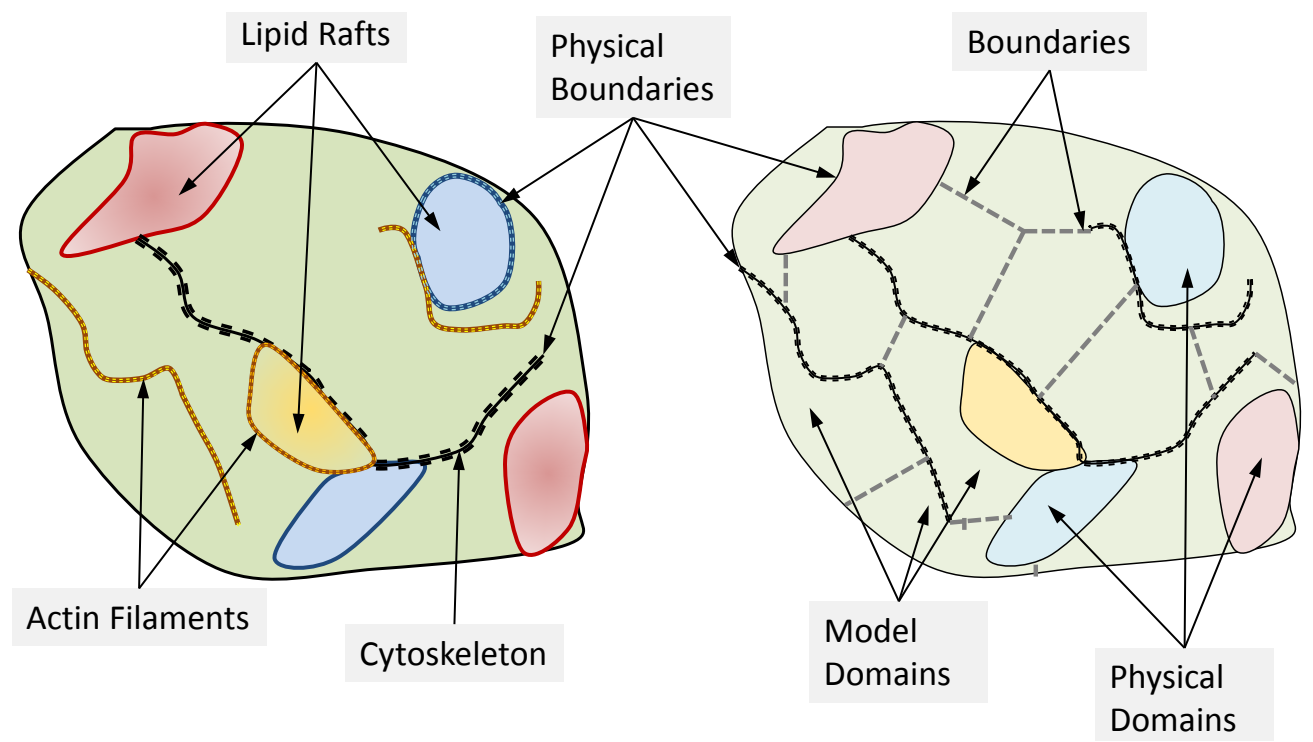

Figure 24: The attractive microdomains are part of a diverse membrane landscape and represent a small fraction of its area. For the purpose of modeling, (1)we assume that the attractive domains are approximately of the same size and (2) we partition the rest of the membrane into domains of comparable size. Diagram following [24]

Attractive domains as high affinity boxes. Using this hypothesis, we developed a simple model to predict the way the observed points group into clusters. We assume that the area observed in each micrograph contains a number of pre-existing attractive domains, and that each domain is approximately the same size. These domains are represented by 'boxes' that will have a high affinity to the diffusing receptors. If we know the number of high affinity boxes $\left(N_{B H}\right)$, the simplest approach would be to take the number of points observed in a given micrograph $(R)$, and calculate the most likely numbers of points in each box, assuming random placement ${ }^{2}$. The points in a box correspond to a cluster, and the predicted particle numbers per box would be compared to the observed number of poins per cluster.

Incomplete confinement of particles and low affinity boxes. Attempts to fit this model to cluster distributions of individual micrographs quickly fail, because we can not simultaneously account for the larger clusters of more than 5 particles and the significant number of particles that are in a cluster by themselves (see Fig. 23). Random placement of $R$ particles into $N_{B H}$ boxes results in distributions that underestimate either the lower end (for small $N_{B H}$ ) or the higher end (for large $N_{B H}$ ) of the distribution. To correct this, we must account for the possibility that a fraction of the particles may remain outside the attractive domains; this fraction needs to be identified as a second model parameter.

\footnotetext{
${ }^{2}$ we will do this next, using a multinomial distribution
} 
There is one more issue with the particles outside attractive domains. Counting them all as singletons misses small clusters of 2-3 receptors, which may be the result of random co-location outside an attractive domain. To account for this, we imagine we subdivide the rest of the cell membrane into regions ("virtual domains") of the same size as the high affinity domains. Figure 24 illustrates the process. The true attractive domains (shown in color) have physical boundaries that control the movement of particles; the domains resulting from a formal subdivision of the rest of the cell membrane may have some physical boundaries, but the movement of particles between these domains is essentially unimpeded. We will treat these new domains as low affinity boxes, that have the same size as the $N_{B H}$ previously defined high affinity boxes. Particles will also be placed at random into these domains, but with a lower probability.

\subsubsection{Model Definition}

There are a number of number of high density boxes $\left(N_{B H}\right)$ which correspond to attractive microdomains in a membrane patch of area $A_{T}$. The total area of all the micro-domains put together is $A_{H}$, therefore they occupy a fraction $f=\frac{A_{H}}{A_{T}}$ of the membrane. The rest of the membrane area, $A_{L}=A_{T}-A_{H}$, is subdivided into $N_{B L}$ domains called "low affinity boxes". These boxes have the same individual size as their high affinity counterparts ${ }^{3}$. The number of boxes of each type is approximately proportional to the corresponding areas, $N_{B H} / N_{B L} \approx A_{H} / A_{L}$.

The physical property of microdomains that causes them to accumulate receptors is quantified by attractiveness, $\alpha$. We define $\alpha$ as the ratio between the equilibrium density of receptors inside versus outside attractive domains. The model is defined by three parameters, $\alpha$, the total number of boxes $N_{B}=N_{B H}+N_{B L}$, and the fraction of area (and boxes) that corresponds to the high affinity domains, $f=N_{B H} / N_{B} \approx A_{H} / A_{T}$. The triplet $\left(\alpha, f, N_{B}\right)$ fully defines one instance of the model that will be fitted to cluster distributions. The number of boxes of two types is obtained as follows,

$$
N_{B H}=f \cdot N_{B} ; N_{B L}=(1-f) \cdot N_{B}
$$

Note that an instance of the model represents an area of the membrane corresponding to one micrograph. The parameters are not identical, but are closely related to physical properties of the membrane, namely, the size, density, and receptor affinity of attractive domains. We expect these properties to vary due to biological factors such as the experimental condition and the part of the membrane that is imaged, but also expect the same set of parameters to reasonably match groups of several images.

\footnotetext{
${ }^{3}$ As we discussed, this division is artificial and is meant to represent the fact that clusters are identified based on distance. Our clustering methods, even if it works perfectly, will identify a group of receptors co-located in an area of the typical size of an attractive domain as a "cluster", whether or not they are in a special domain or they just happen to be co-located.
} 

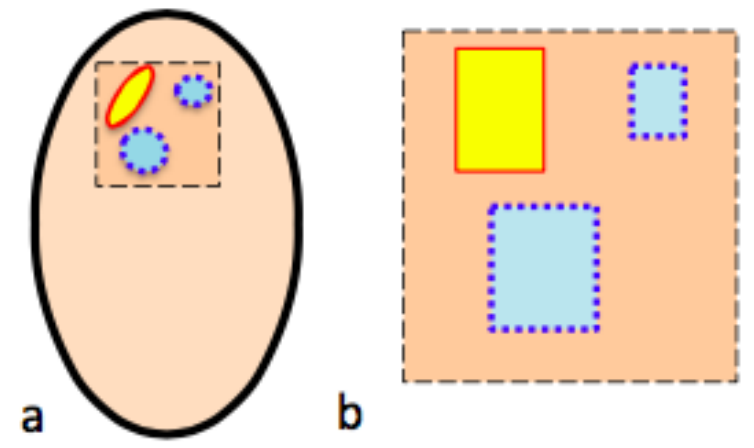

b

Figure 25: Attractive microdomains occupy a small fraction of the cell membrane, and their measurements may be extracted from experimental images. Diagram from [3].
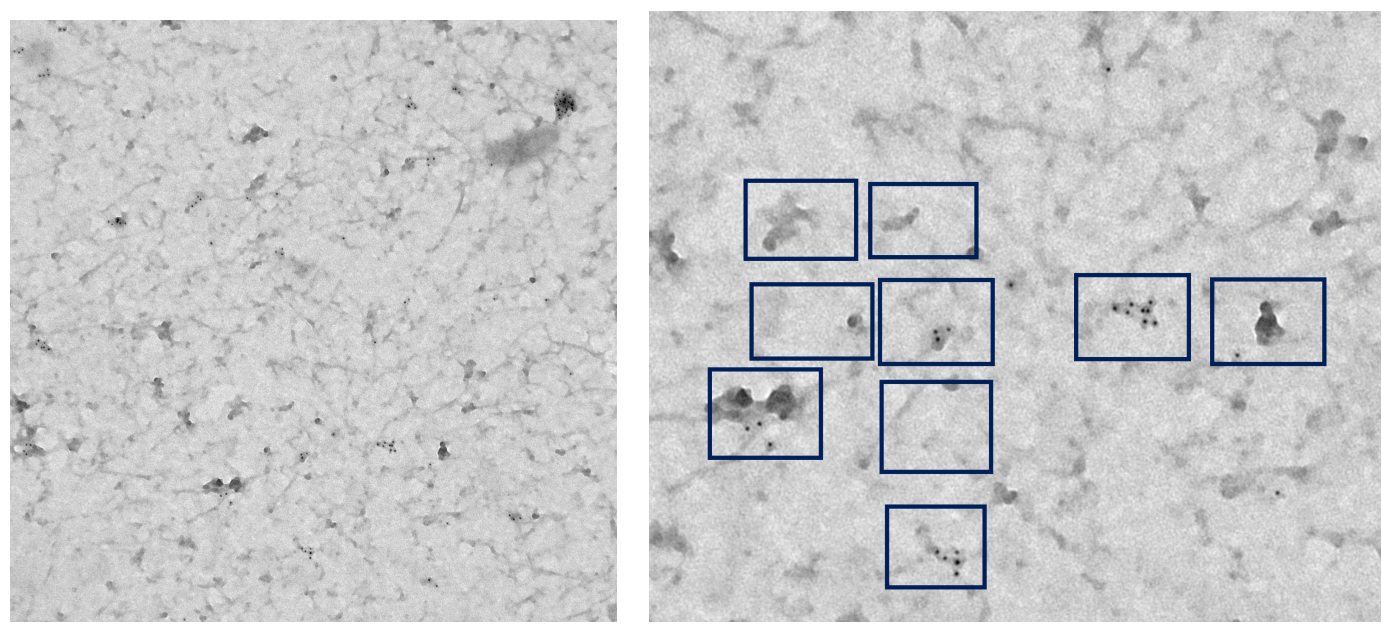

Figure 26: Micro-domains and our model. 

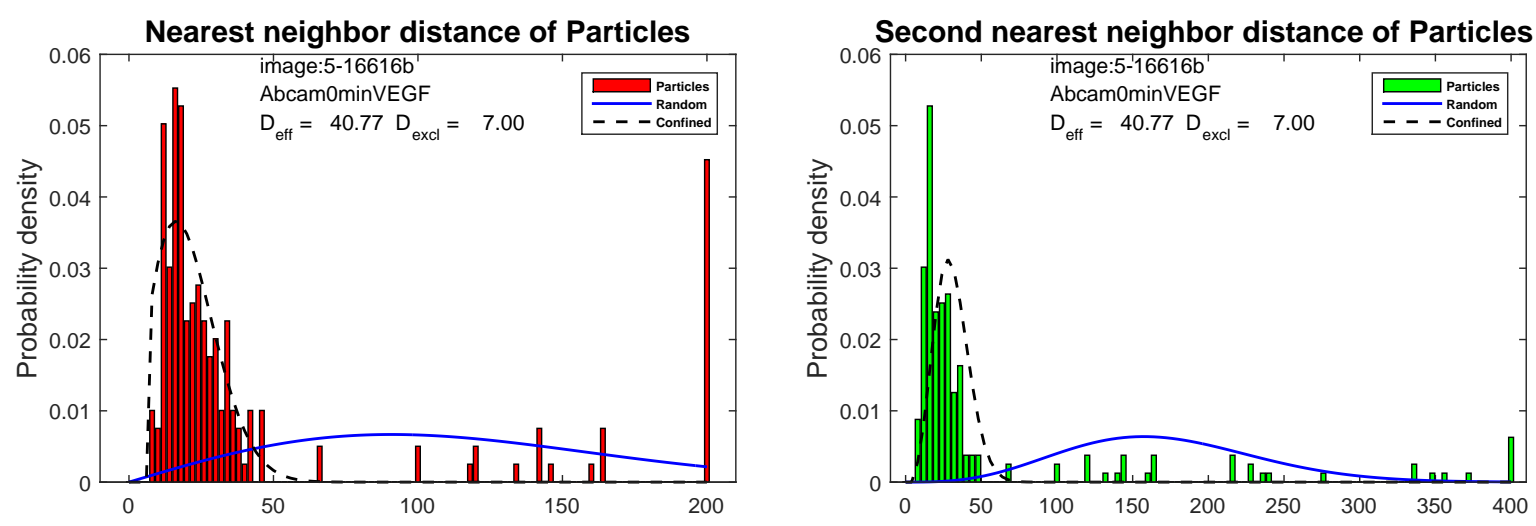

Figure 27: Nearest and next nearest neighbor distributions of image $5-16616 b$ Abcam 0 minutes. The short distance part is consistent with random placement in an area comparable to that of the clusters. The few large values correspond to single-particle clusters, and they ar distributed consistently with the random curve correspoinding to the entire image area.

\subsubsection{Understanding the Spatial Distribution other than Microdomain-Induced Clustering}

The model as defined in Section 2.1.2 does not explicitly refer to the exact location of the receptors. We previously used various statistics to establish the fact of clustering, but a complete understanding of the phenomena behind clustering should provide some insight into how the observed spatial distributions emerge.

The main hypothesis needs to be complemented to address the detailed distribution of receptors. Here we will focus on the simplest possible additional assumption, that other than the accumulation of receptors inside attractive domains, the distribution of receptors within and outside domains, as well as the location of the domains themselves, are all uniform and random.

This assumption implies that there is no additional structure to the receptor distributions other than microdomain-induced clustering. This additional hypothesis can be tested by attempting to deconstruct the previously discussed spatial distributions into parts that reflect the distribution of particles inside clusters and the distribution of cluster locations.

\section{Localization of Cluster Centroids}

Similar to particle distributions within a domain, we obtained the distribution of clusters for a given cell.To understand whether the location of centroids (center of each cluster) are random or not, we apply the same set of methods and calculations as for particle distributions. We take the cluster 
centroids this time and apply our probability calculations to them. As in particles distribution with a domain, our hypothesis is that centroids are localized randomly on the membrane domain.

\subsubsection{Predicting the Distribution of the Number of Particles per Cluster}

The attractive domain hypothesis implies that the majority of the observed clusters correspond to an attractive domain. Assuming the domains are approximately the same size, the distribution of the observed particles among these domains should follow a random (multinomial) distribution. In addition to the clusters corresponding to attractive domains, we estimate the likelihood that clusters emerge randomly among the rest of the particles that are not in the attractive domains.

The model defined in Section 2.1.2 randomly places $R$ receptors into a set of $N_{B}$ boxes that are divided into two types, $N_{B H}$ high affinity and $N_{B L}$ low affinity. This is a stochastic model which has a number of possible outcomes (states), characterized by the number of receptors placed in each box. The boxes of each type are identical, and outcomes are usually grouped by the number of boxes that have $0,1,2, \cdots$ receptors.

Each run or instance of the model produces a specific outcome. We implemented a computer simulation of the model that can be used to estimate the probability of individual outcome (types). This is used in conjunction to analytical calculations (presented later) of expected cluster distributions. The full model prediction would be a set of probabilities assigned to each individual outcome (or outcome group). In this work, we compare the average number of occurrences of $k$ receptors in a box to the number of observed clusters of size $k$.

\subsection{Spatial Distribution}

\subsubsection{Analysis of Distribution within Clusters}

We constructed clusters for values of the distance parameter ranging from $0.698 \mathrm{~nm}$ (1 pixel) to a few hundred, in order to obtain the full $N_{\mathrm{C}}(L)$ dependence. We can visualize the distribution of clusters for various length scales. A detail of the cluster map by using our domain construction algorithm shown in Figure (28). The colors are used to indicate the different clusters.

The dependence of the number of clusters on the length scale is plotted in Figure (29), for a single image as well as for the entire set analyzed. We first scaled the theoretical (random) distribu- 

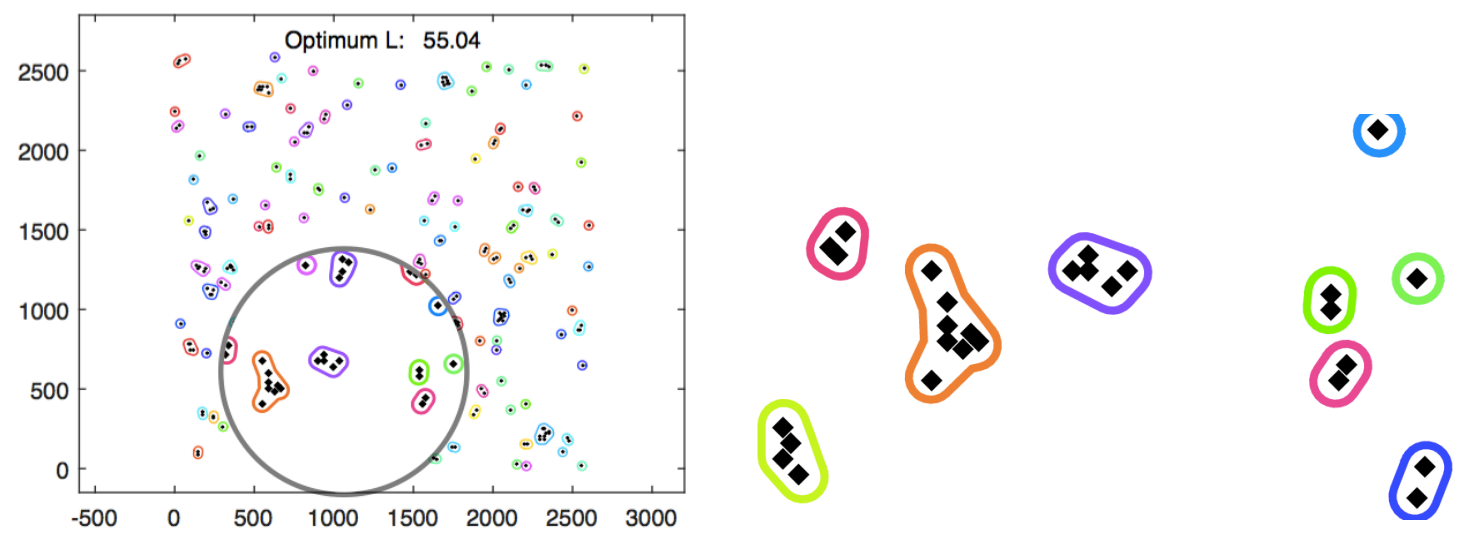

Figure 28: Left: Cluster or contour map of image no: 516646 by using domain construction algorithm. Disks corresponding to the same cluster have the same color. Right: Detail of the cluster map.

tions to correspond to a higher particle density $D_{\text {eff }}$. We then added a correction to take into account a minimal separation of points, by setting the corresponding pdf $p(x)$ to zero for $x \leq D_{\text {exclusion }}$ in the case of nearest neighbor distance distributions, and by shifting the entire cluster size distribution by the exclusion distance $D_{\text {exclusion. }}$. Finally, the curves were scaled vertically to match the integral of the high density mode (NND), respectively the total number of points (clustering). Comparison with the random distribution shows clear and consistent deviations. Both the individual image and the cumulative plot exhibit a sharp initial decrease, followed by a significantly slower variation. The initial fast decrease corresponds to the fact that particles are much closer to their neighbors than the average distance. In an ideal clustered scenario, where the intra-cluster separation between particles is smaller than the shortest distance between clusters, the number of clusters would decrease until the largest intra-cluster distance, and one would then observe a plateau until $L$ becomes comparable to the inter-cluster distance. While we do not observe a perfect scale separation, both the single image and the cumulative curves exhibit a clear change in behavior around $27.93 \mathrm{~nm}$ (40 pixels), and a shoulder that extends to approximately $48.89 \mathrm{~nm}$ (70 pixels).

\subsubsection{Analysis of Distribution Between Clusters}

After analyzing the distribution of particles within the clusters, next thing to understand is the distribution and localization of centroids which is taking each cluster on a given image as a particle in the given area. We find the center of each cluster by taking the average of particles coordinates location of each cluster which we call it a centroid. We then analyze the distribution of centroids as the same way we did for particles within the clusters. We used the nearest neighbor distribution, 

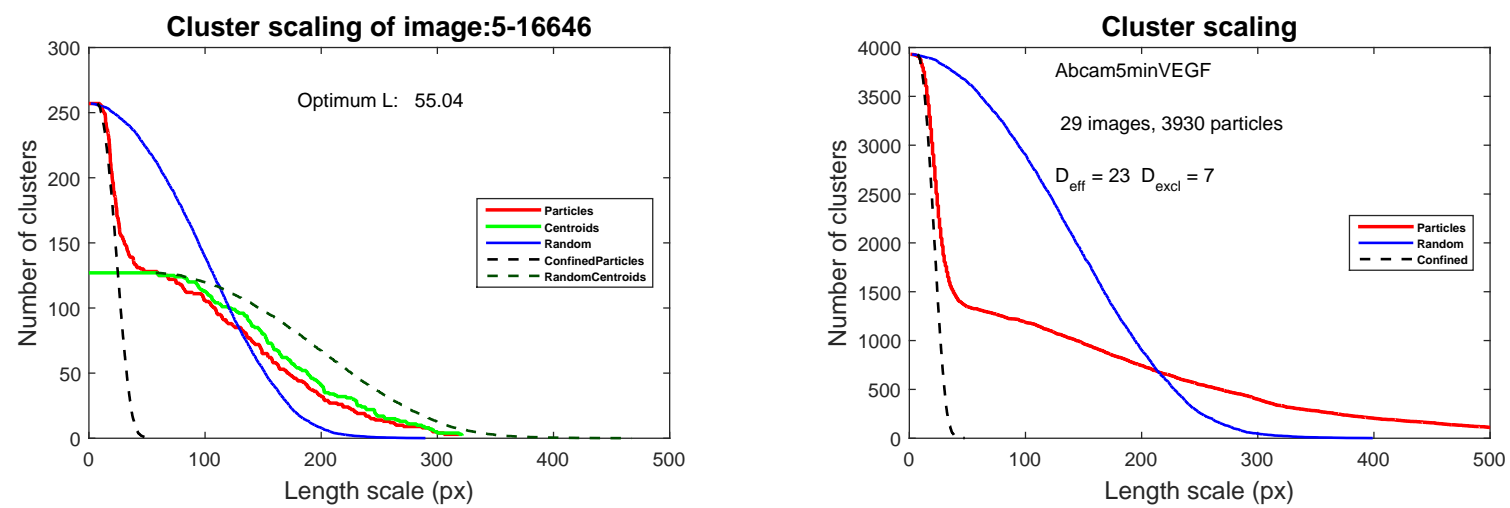

Figure 29: Number of clusters as a function of the length parameter (red lines) in a single TEM image (left), and a set of 28 images (right), compared to the average number of clusters expected from a random distribution with the same number points in the same area (blue lines).

Hopkins and Ripley statistic tests to analyze the clustering formation. These methods can distinguish between more and less clustered data even though they do not provide a simple quantitative measure of the extent of clustering. The reason the need of a new and simple predictive model is to provide the quantitative measurements by quantifying both clustering and the effect of microdomains on clustering amount. As in shown Figure (29) on the left the green curve corresponds to the distribution of centroids of the given length scales. The result of centroid distribution is consistent with the distribution of particles within the clusters.

\subsection{Number of Particles by Cluster}

\subsubsection{Distribution of Domain Sizes and Shapes}

The decision on the characteristic length based on hierarchic clustering distance facilitate combination and comparison of a number of calculations. The domain reconstruction algorithm compiles the particles. The experimental data is then converted to static spatial data and used to determine domain size and distribution. The domains are reconstructed via a clustering algorithm, contour drawing. Because we now have the contours or in other words clusters which reveals us to calculate total area, perimeter and give the knowledge of even the shapes of clusters. The domain reconstruction algorithm converts particle clustering to static spatial data that can be used to approximate the size and contours of confinement zones occupied by VEGF ligands on the cell membranes. The algorithm is fully described in Chapter 2. 

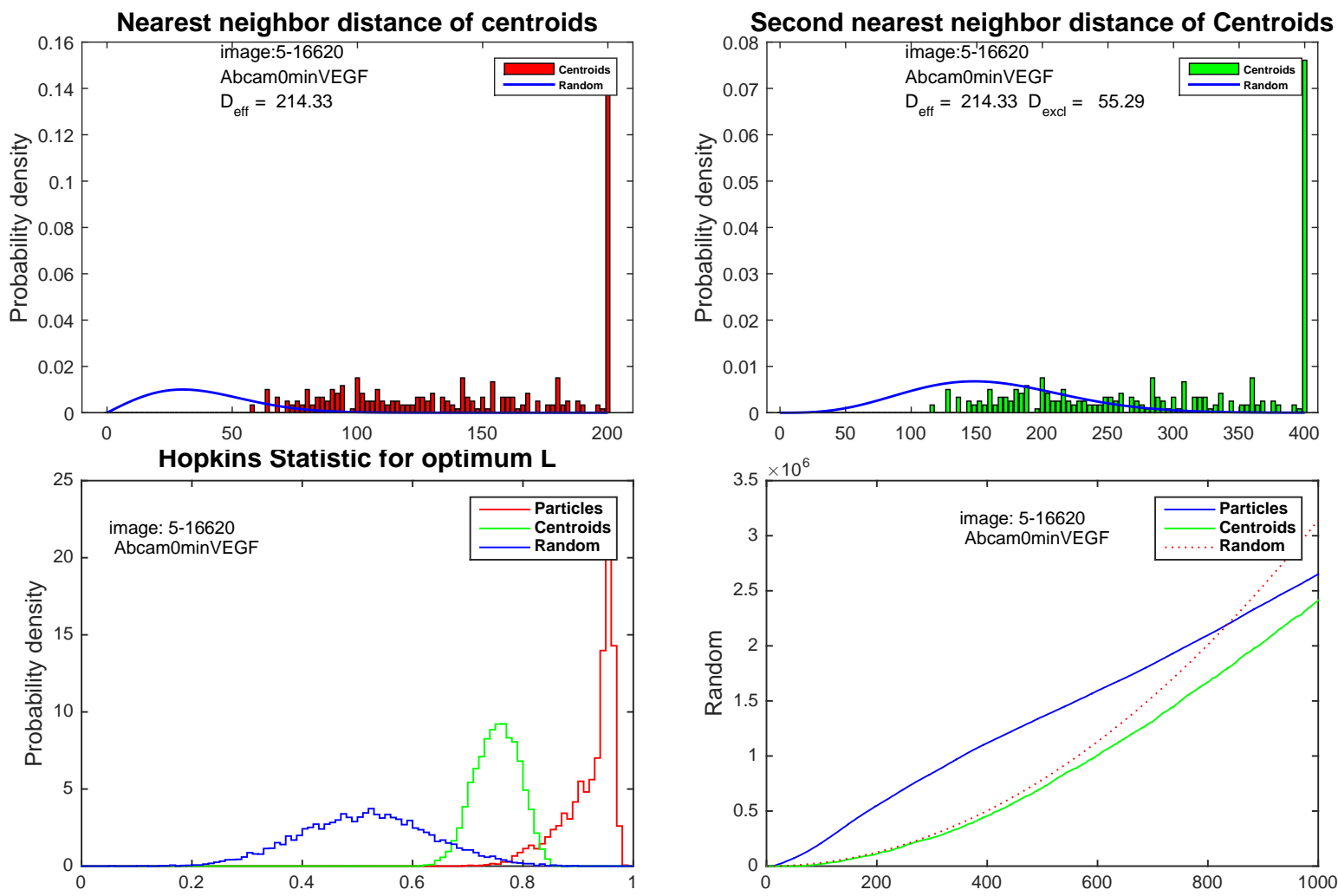

Figure 30: Statistical measures of clustering applied to the cluster centroids for image $5-16620$. The plots are not fully consistent with random placement, but are closer to the random expecation than the corresponding statistics of the particle distributions. 
Based on area, perimeter and cluster sizes comparisons we can group TEM images on common properties. This is a very important finding to analyze clustering phenomenon of VEGF receptors quantitatively. Having the quantities of domain sizes and shape, we compare the domain size and shapes with the clusters. The results of distribution of domain sizes and shapes which is derived by reconstruction of the domains shows consistency with the clusters on TEM images.

\section{Number of Particles, Clusters and the Optimal Clustering Length of Images}

By using distance based hierarchic clustering, we identified the number of clusters and characteristic length of images with a given number of particles. One way to approach the clustering phenomenon is analyzing the data image by image then comparing the set of data for resting and activated cells. We also analyzed the characteristic properties of each data set by comparing two data subsets; Abcam and CS for different stimulus time durations. Grouping somewhat similar objects based on those characteristics information is another approach. Therefore, we perform histograms on resting and stimulated cells with the number of particles, characteristic lengths, and number of clusters in order to understand the characteristics of each data set. As it was shown in Fig.13, even though there are a number of outliers in the histograms of number of particles, optimum length scales and number of clusters versus the number of images, one observations instant observation is that the majority of the images are distributed normally with the corresponding parameters scales.

\section{Aggregate Area of Domains as defined by the Domain Reconstruction Algorithm}

We determine the characteristic length which best describes the distance between points in a cluster. This length is varied from 0 pixels to 500 pixels. Over this range the average length of the clusters is computed. The length between two particles reaches a local maximum over this range where is used as the characteristic length. It is then used to create the clusters to construct a contour that will define the final confinement area. The contour is created by inflating the cluster. The outside points of the cluster are extended outward by $\frac{1}{2}$ the characteristic length. This allows some of the domains give in the confinement area to consider that these points could have fluctuated a structure and would not have sat directly next to the confining structure. The extended points are then connected to create a contour which is the final domain structure. The reconstructed domains are statistically different. 

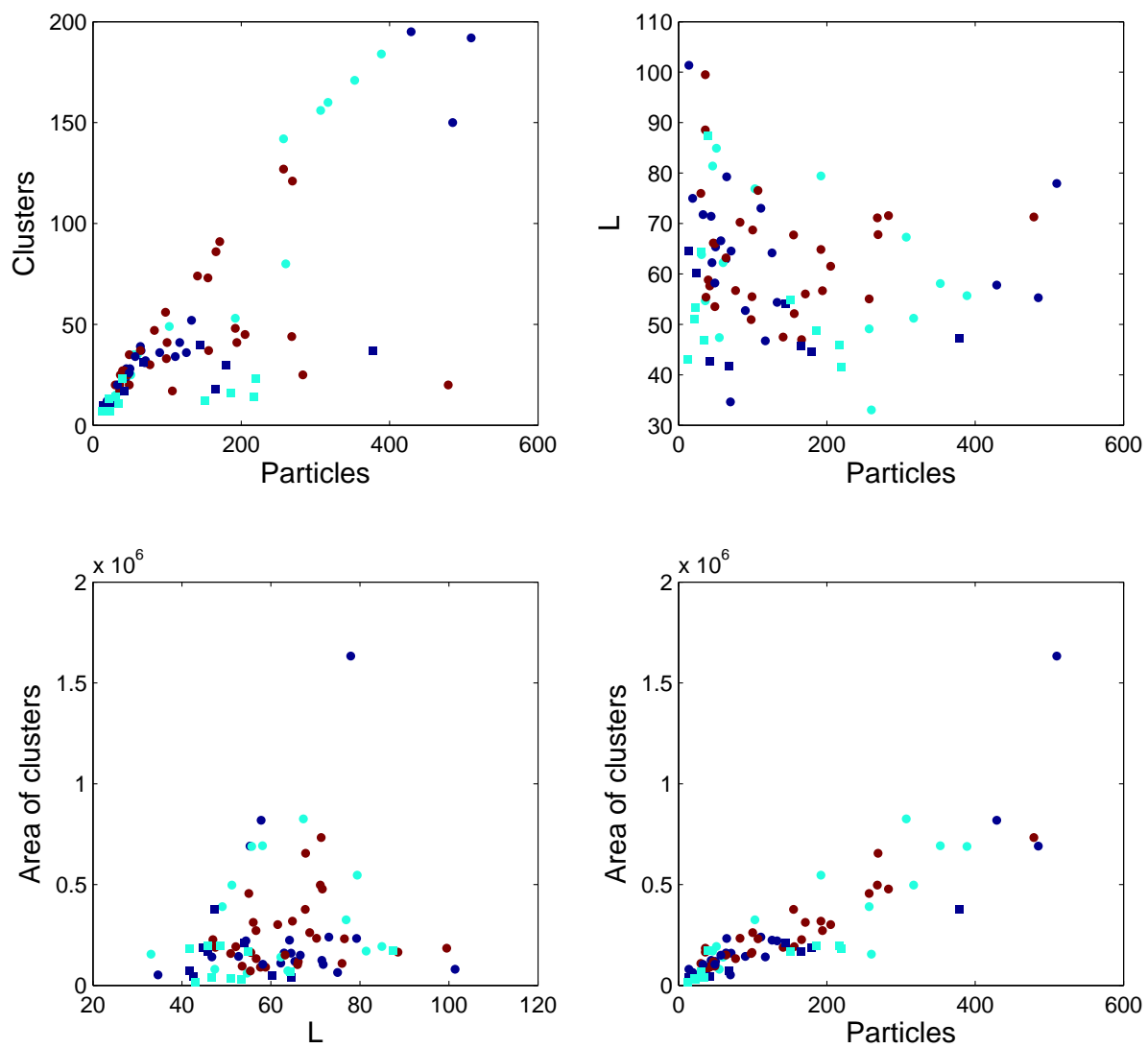

Figure 31: Scatter plots of the characteristics of clusters for all Abcam resting cells.

\section{Area and Perimeter of Clusters}

By using the domain reconstruction algorithm, we can now calculate the area and perimeter of the polygons. We observed $P^{2} / A / N_{C}$ value which we call form factor is important because this value gives an idea about the shape of the clusters in a given micrograph. As it is shown in Fig.32, the majority of clusters are shaped as circle where as there are outliers that are not close to a circle shape. 

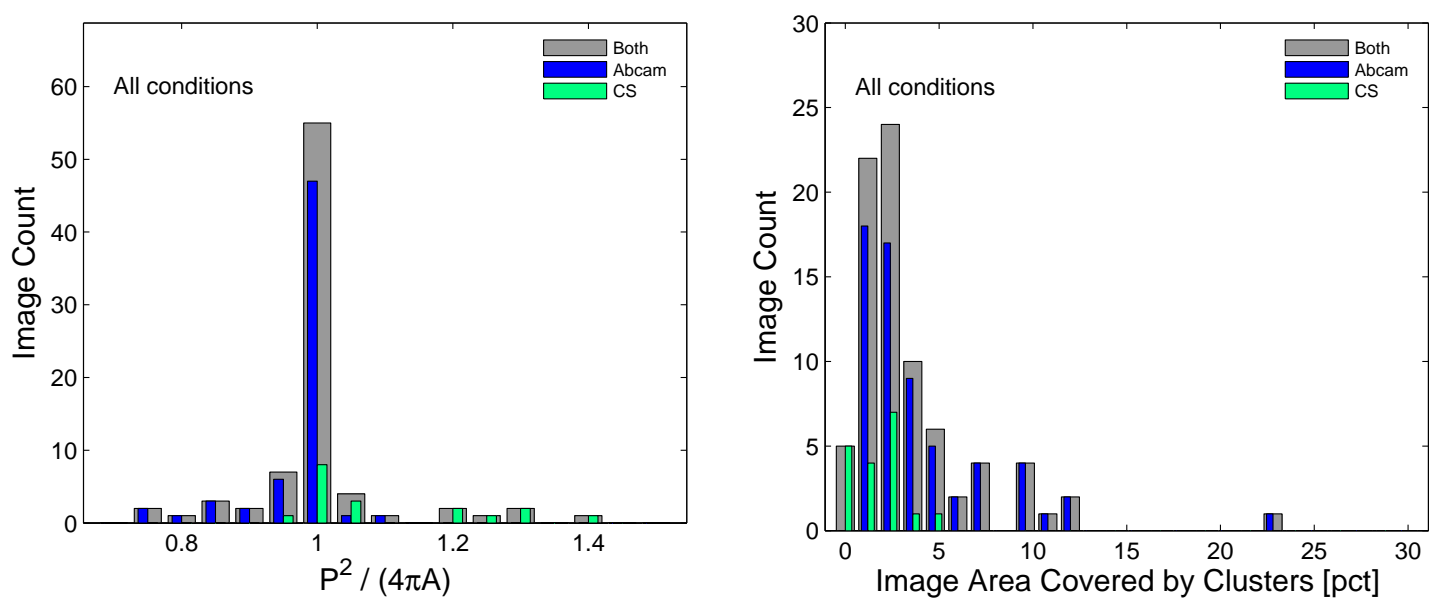

Figure 32: Histograms of all the data sets that decribes the properties of the clusters such as area, perimeter and the shape.

\subsection{Fitting the Stochastic Model of Cluster Distribution}

\subsubsection{Model Definition}

Assuming that we have identified the clusters in a set of images, we can use the distribution of cluster sizes to test our hypotheses regarding the mechanism behind clustering. Below we outline a predictive model for cluster distributions based on the hypothesis of pre-existing high affinity micro-domains. These are small areas in the cell membrane that are attractive to receptors, in the sense that receptors spend a relatively high fraction of their time in these regions. Such attractive areas could be, for example, lipid rafts, protein islands, or regions immediately adjacent to elements of the cytoskeleton.

A number of $R$ particles (receptors) are placed randomly in $N_{B}$ compartments (boxes). There are two types of boxes, high affinity (or "high density"), and low affinity. We denote the fraction of HD boxes by $f$, so the numbers of HD and LD boxes are respectively

$$
N_{B H}=f \cdot N_{B} ; N_{B L}=N_{B}-N_{B H}=(1-f) \cdot N_{B} .
$$

Particles may locate in any box, independently of the number of particles already in there. The probability that a specific particle falls into a specific box is the same as that of falling into any other box of the same type. We will sometimes refer to the entirety of boxes of one type as the "HD (or LD) sector". The probability that a (specific) particle falls into a (specific) HD box is $\alpha$ times higher than that of falling into a LD box.

$$
p_{H}=\alpha \cdot p_{L}
$$


We are interested in the distribution of the number of particles per box, to be compared to the distribution of the number of observed particles per cluster. Our goal is to identify a set of model parameters $\left\{\alpha, N_{B}, f\right\}$ that work (i.e. provide a reasonable match) for a large set of images, irrespective of the number of receptors captured in the image.

We will now try to derive a simple prediction for the distribution of the number of particles per box. The probabilities defined in Eq. 2.3 refer to a single box of the respective types. The probability that a given particle falls into any $\mathrm{HD}$ box is $N_{B H} . p H$, and the corresponding probability for any LD box is $N_{B L} \cdot p_{L}$. These two must add up to 1 , since they are mutually exclusive and there are no other possibilities. Based on this normalization argument, we can derive the actual values of $p L, p H$ :

$$
\begin{array}{r}
1=N_{B H} \cdot p_{H}+N_{B L} \cdot p_{L}=N_{B} \cdot(f \cdot \alpha+(1-f)) \cdot p_{L} \Rightarrow \\
p_{L}=\frac{1}{N_{B}(\alpha f+1-f)} ; \quad p_{L}=\frac{\alpha f}{(\alpha f+1-f)}
\end{array}
$$

The probabilities that a particle flaunt either type of box are;

$$
p_{L}^{(t o t)}=\frac{1-f}{\alpha f+1-f} ; p_{L}^{(t o t)}=\frac{\alpha f}{\alpha f+1-f}
$$

and we use them to define the expected number of particles in each sector,

$$
R_{H}=R \cdot p_{H}^{(t o t)}=R \cdot \frac{\alpha \cdot f}{\alpha f+1-f} ; R_{L}=R \cdot p_{L}^{(t o t)}=R \cdot \frac{1-f}{\alpha f+1-f}
$$

Define the average number of particle per box $\lambda=\frac{R}{B}$ for the system, and for each sector:

$$
\lambda_{H}=\frac{R_{H}}{N_{B H}}=\frac{\lambda \alpha}{\alpha f+1-f} \cdot \lambda_{L}=\frac{R_{L}}{N_{B L}}=\frac{\lambda}{\alpha f+1-f}
$$

Distributing exactly $R$ particles in the manner described above corresponds to a "random experiment" that has a large number of possible outcomes, identified by the number of particles in each box,

$\left\{r_{H, 1}, r_{H, 2}, \cdot, r_{H, N_{B H}} ; r_{L, 1}, r_{L, 2}, \cdot, r_{L, N_{B L}}\right\}$, where the number of particles in each sector,

$$
R_{H}^{*}=\sum_{j=1}^{N_{B H}} r_{H j} ; \quad R_{L}^{*}=\sum_{j=1}^{N_{B L}} r_{L j}=R-R_{H}^{*}
$$

is also the result of random sampling. The distribution of $R_{H}^{*}$ is binomial, with $R$ trials and probability $p_{H}^{(t o t)}$. Then, for each $R_{H}^{*}$, we have to distribute the $R_{H}^{*}$ particles into the $N_{B H}$ boxes. The distribution of the number of particles that fall into each box is multinomial, with $R_{H}^{*}$ trials and $N_{B H}$ possible outcomes each with probability $p=1 / N_{B H}$. This is a complicated way to obtain a result that is well approximated by a simpler argument. 
The simpler approach is as follows. Once we identify $\lambda_{H}$ and $\lambda_{L}$, we can imagine that each box (which represents a small area on a relatively very large cell) receives particles from a very large number of "trials", in a way that average number of particles per box is set by the corresponding $\lambda$. The probability that exactly $k$ particles of the $N$ total fall into this box is controlled by a binomial with success probability $\lambda / N$ and $N \gg \lambda$ trials:

$$
\begin{aligned}
P(k, \lambda ; N) & =\left(\frac{\lambda}{N}\right)^{k} \cdot\left(\frac{N-\lambda}{N}\right)^{N-k} \cdot\left(\begin{array}{c}
N \\
k
\end{array}\right) \\
& =\frac{\lambda^{k}}{k !} \cdot \frac{N \cdot(N-1) \cdot \cdot(N-k+1)}{(N-\lambda)^{k}} \cdot\left(1-\frac{\lambda}{N}\right)^{N}
\end{aligned}
$$

The limit of $P(k, \lambda ; N)$ as $N \rightarrow \infty$ works out a Poisson distribution,

$$
\varphi_{k}(\lambda)=\lim _{N \rightarrow \infty} P(k, \lambda ; N)=e^{-\lambda} \cdot \frac{\lambda^{k}}{k !}
$$

Assuming that the $N_{B H}$ HD boxes receive particles independently of each other and of the LD boxes (which amount to the same hypothesis as above), the average number of HD boxes that contain exactly $k$ particles, irrespective of each sector, is

$$
b_{k}^{(t o t)}=R_{H} \cdot \varphi_{k}^{\left(\lambda_{H}\right)}+R_{L} \cdot \varphi_{k}^{\left(\lambda_{L}\right)},
$$

where $R_{H}, R_{L}$ and $\lambda_{H}, \lambda_{L}$ are given by Eqs. $(2.5,2.6)$. Let the area of a cell be $A$. Assuming the colored regions in Fig. 25 that may contain clusters be the high density domain, let the area of high density domain be $A_{H}=f A$, and the rest area be $A_{L}=(1-f) A$. For one particle, let the probability that it falls in to $A_{H}$ ( $A_{L}$ respectively) be $p_{H}$ ( $p_{L}$ resp.), and let the attractiveness of high density domain be $\alpha$. Then we have that $\frac{p_{H}}{p_{L}}=\frac{\alpha A_{H}}{A_{L}}$. As $p_{H}+p_{L}=1$, it follows that $p_{H}=\frac{\alpha A_{H}}{\alpha A_{H}+\alpha A_{L}}$. By substituting $A_{H}=f A$ and $A_{L}=(1-f) A$, we have

$$
p_{H}=\frac{\alpha f}{1+(\alpha-1) f} .
$$

Similarly, we have

$$
p_{L}=\frac{1-f}{1+(\alpha-1) f} .
$$

For simplifications, we assume that the whole cell is the replication of one patch (i.e. cell = $Q *$ one patch), and the area of boxes that may contain clusters are the same, denoted as $a_{0}$ (refer to a figure here!!!). We divide a patch to high density bins and low density bins.

Let $N_{P}^{H}$ ( $N_{P}^{L}$ respectively) be the number of particles in high (low resp.) density domain and $N_{B}^{H}$ ( $N_{B}^{L}$ resp.) be the number of boxes in high (low resp.) density domain. 
Then $N_{B}^{H}=\frac{A_{H}}{a_{0}}=\frac{f A}{a_{0}}$ and $N_{B}^{L}=\frac{A_{L}}{a_{0}}=\frac{(1-f) A}{a_{0}}$.

And let $Q_{B}^{H}=Q \cdot N_{B}^{H}\left(Q_{B}^{L}=Q \cdot N_{B}^{L}\right.$ resp.) be the number of boxes in the high (low resp.) density domain a cell, and

$Q_{P}^{H}=Q \cdot N_{P}^{H}\left(Q_{P}^{L}=Q \cdot N_{P}^{L}\right.$ reps.) be the number of particles in high (low resp.) density domain of a cell.

The probability of a specific particle falls into a specific box is

$$
p=\left\{\begin{array}{l}
p_{H} \cdot \frac{1}{Q_{B}^{H}}, \text { if the particle falls in high density region; } \\
p_{L} \cdot \frac{Q_{B}^{H}}{Q_{B}}, \text { if the particle falls in low density region. }
\end{array}\right.
$$

In the following context, we focus on the events in high density domain. The probability of specific $k$ particles falls into a specific box is $p^{k}$. Therefore, the probability that any $k$ particles falls into a specific box is

$$
\left(\begin{array}{c}
Q_{P}^{H} \\
k
\end{array}\right) p^{k}(1-p)^{Q_{P}^{H}-k}=\frac{Q_{P}^{H} !}{\left(Q_{P}^{H}-k\right) !}(1-p)^{Q_{P}^{H}-k} \frac{1}{k !} p^{k}
$$

Assume that the number of particles in our chosen set of $N_{B}^{H}$ boxes is $\left(k_{1}, k_{2}, \cdots, k_{N_{B}^{H}}\right)$ with

$$
\sum_{i=1}^{N_{B}^{H}} k_{i}=N_{P}^{H}
$$

By neglecting the fact that these events are independent since we have a huge number of particles and boxes, one can assume that the probability of a specific configuration is

$$
\begin{aligned}
P_{\mathrm{abs}}\left(k_{1}, \cdots k_{N_{B}^{H}}\right) & =\prod_{i=1}^{N_{B}^{H}}\left(\begin{array}{c}
Q_{P}^{H} \\
k_{i}
\end{array}\right)(1-p)^{Q_{P}^{H}-k_{i}} p^{k_{i}} \\
& =\prod_{i=1}^{N_{B}^{H}} \frac{Q_{P}^{H} !}{\left(Q_{P}^{H}-k_{i}\right) !}(1-p)^{Q_{P}^{H}-k_{i}} \frac{1}{k_{i} !} p^{k_{i}}
\end{aligned}
$$

For big enough numbers of particles we have $\frac{Q_{P}^{H} !}{\left(Q_{P}^{H}-k_{i}\right) !} \approx Q_{P}^{H^{k}}$. Hence,

$$
\begin{aligned}
P_{\mathrm{abs}}\left(k_{1}, \cdots k_{N_{B}^{H}}\right) & \approx(1-p)^{Q_{P}^{H}} \prod_{i=1}^{N_{B}^{H}} \frac{Q_{P}^{H_{i}^{k}}}{k_{i} !} \cdot\left(\frac{p}{1-p}\right)^{k_{i}} \\
& =Q_{P}^{H^{N_{P}^{H}}}(1-p)^{Q_{P}^{H}}\left(\frac{p}{1-p}\right)^{N_{P}^{H}} \prod_{i=1}^{N_{B}^{H}} \frac{1}{k_{i} !} .
\end{aligned}
$$


Substituting (2.11), $Q_{P}^{H}=Q N_{P}^{H}$ and $Q_{B}^{H}=Q N_{B}^{H}$ to (2.15), for the case of $p=p_{H} \cdot \frac{1}{Q_{B}}$, we have that

$$
\begin{aligned}
& (1-p)^{Q_{P}}=\left(1-\frac{P_{H}}{Q N_{B}}\right)^{Q N_{P}}=e^{-\frac{N_{P}}{N_{B}} p_{H}} \text { as } Q \rightarrow \infty \\
& \text { and } \frac{p}{1-p}=\frac{p_{H}}{Q_{B}} /\left(1-\frac{p_{H}}{Q_{B}}\right)=\frac{p_{H}}{Q_{B}-p_{H}} \simeq \frac{p_{H}}{Q_{B}} .
\end{aligned}
$$

Therefore, (2.15) can be simplified to the following

$$
P_{\text {abs }}^{H}\left(k_{1}, \cdots, k_{N_{B}^{H}}\right) \approx\left(\frac{N_{P}^{H}}{N_{B}^{H}}\right)^{N_{P}^{H}} p_{H}^{N_{P}^{H}} e^{-\frac{N_{P}}{N_{B}} p_{H}} \prod_{i=1}^{N_{B}^{H}} \frac{1}{k_{i} !} .
$$

This is the absolute probability of having $\left(k_{1}, \cdots, k_{N_{B}^{H}}\right)$ particles in the high density domain (or low density domain) in our favorite patch of $N_{B}^{H}$ bins. Now, there is no guarantee that this patch will receive exactly $N_{P}^{H}$ particles.

The probability of a specific particle falling into our set of $N_{B}^{H}$ bins is $p_{\text {patch }}=N_{B}^{H} / Q_{B}^{H}=1 / Q \ll$ 1 .

Then, the probability of any $N_{P}^{H}$ particles in this patch is

$$
\begin{aligned}
P_{\mathrm{patch}}\left(N_{P}^{H}\right) & =\left(\begin{array}{c}
Q_{P}^{H} \\
N_{P}^{H}
\end{array}\right) p_{\mathrm{patch}}^{N_{P}^{H}}\left(1-p_{\mathrm{patch}}\right)^{Q_{P}^{H}-N_{P}^{H}} \\
& \approx Q_{P}^{H_{P}^{N_{P}^{H}}}\left(1-p_{\mathrm{patch}}\right)^{Q_{P}^{H}}\left(\frac{p_{\mathrm{patch}}}{1-p_{\mathrm{patch}}}\right)^{N_{P}^{H}}
\end{aligned}
$$

We are looking for the relative probability of a specific $\left(k_{1}, \cdots, k_{N_{B}}\right)$ that satisfies (2.13),

So we should divide (2.15) by (2.17). The large factor $Q_{P}^{N_{P}}$ cancels out.

Then $(1-p)^{Q_{P}}$ type factors can be approximated as follows Since we know that

$$
\begin{gathered}
(1-p)^{Q_{P}} \approx \lim _{Q \rightarrow \infty}\left(1-\frac{1}{Q N_{P}}\right)^{Q N_{P}} \rightarrow e^{-1} \\
\left(1-p_{\text {patch }}\right)^{Q_{P}} \approx \lim _{Q \rightarrow \infty}\left(1-\frac{1}{Q N_{B}}\right)^{Q N_{P}}=e^{-N_{P} / N_{B}} \text { etc. }
\end{gathered}
$$


follows from (2.18)

As $p<<1$ and $p_{\text {patch }}<<1$, we have that $1-p \approx 1$ and $1-p_{\text {patch }} \approx 1$. Then

$$
\left(\frac{1-p}{1-p_{\text {patch }}}\right)^{Q_{P}} \approx 1
$$

and

$$
\left(\frac{p}{1-p}\right)^{N_{P}} /\left(\frac{p_{\text {patch }}}{1-p_{\text {patch }}}\right)^{N_{P}}=\left(\frac{p\left(1-p_{\text {patch }}\right)}{(1-p) p_{\text {patch }}}\right)^{N_{P}} \approx\left(\frac{p}{p_{\text {patch }}}\right)^{N_{P}}
$$

Substitute (2.11) and $p_{\text {patch }}=\frac{1}{Q}$ to (2.21) and simplify it, we have that

$$
\left(\frac{p}{p_{\text {patch }}}\right)^{N_{P}}= \begin{cases}\left(\frac{p_{H}}{N_{B}}\right)^{N_{P}}, & \text { if in HD region; } \\ \left(\frac{p_{L}}{N_{B}}\right)^{N_{P}}, & \text { if in LD region. }\end{cases}
$$

Therefore, divided (2.15) by (2.17) and combining (2.19), (2.20) and (2.22), we will have the relative probability of a specific $\left(k_{1}, \cdots, k_{N_{B}}\right)$ that satisfies $(2.13)$ is approximately equal to the following

$$
P\left(k_{1}, k_{2}, \cdots, k_{N_{B}}\right)=\left\{\begin{array}{l}
e^{\frac{1}{N_{B}}-1}\left(\frac{e \cdot p_{H}}{N_{B}}\right)^{N_{P}} \prod_{i=1}^{N_{B}} \frac{1}{k_{i} !}, \text { if in HD } \\
e^{\frac{1}{N_{B}}-1}\left(\frac{e \cdot p_{L}}{N_{B}}\right)^{N_{P}} \prod_{i=1}^{N_{B}} \frac{1}{k_{i} !}, \text { if in LD. }
\end{array}\right.
$$

\subsubsection{Fitting the Model to the Data}

Our goal is to identify the model parameters for all data sets both globally and individually. The distribution of the labeled receptors is not uniform. We identify receptor clusters through a hierarchic distance based algorithm with a globally optimized characteristic length, and then summarize the distribution of cluster sizes. We used the hypothesis of attractive micro-domains to build a mathematical model, which provides the probability distribution of cluster sizes. The model parameters are related to the typical size and density of the domains and the relative affinity of the receptors for them. These parameters are not directly measurable. The size of a cluster in an image depends on the number of receptors present in the imaged area, as well as on the labeling efficiency; both may vary substantially. Our approach relies on comparing the model prediction for the cluster size distribution in each image with the one derived experimentally. A MetropolisHastings (simulated annealing) algorithm is used to minimize the overall square distance between the model and experimental distributions. 
In our model, we have has some intrinsic parameters, which we believe reflect the way we think the observed receptors behave, but each file has a different number of points. The distribution of cluster sizes depends on this, also. For instance, even if the intrinsic model parameters are the same for two data or a TEM image, if one data has a lot of points, most of its points are not in small clusters. Whereas, if the other file has only a few points, most of them will be in small clusters.

In principle, each file could follow a separate distribution. On the other hand, some of the files are similar to each other, so they may follow the same distribution. If two files have the same intrinsic parameters but very different numbers of points, the distributions will not be similar. The issue of fitting then revolves around the following: 1) fitting each file with a set of parameters, or 2) obtaining a single set for several files.

\section{Image by image}

The high density model described above only explains the first mode of the observed cluster number distributions and of the nearest neighbor distance plots. The rest of the distribution is somewhat similar to a lower density random distribution, that may reflect the inter-cluster spacing. Our investigation is attempt to reproduce both modes, based on a model of randomly distributed clusters, consisting of particles randomly distributed at a high density. The typical size of a cluster could be inferred from the distribution of the number of particles in a cluster.

We make two assumptions which is consistent with experimental picture. The first assumption is that a fraction $f \leq 1$ of the membrane is covered by domains that have a physical affinity for receptors. As receptors diffuse throughout the membrane, the probability of crossing the boundary of such an attractive domain is asymmetric, all else being equal, inbound crossing is $\geq 1$ times more likely than outbound. We will consider the aggregate of the high affinity patches as a single high density domain, and refer to the rest as the low density domain or sector. Second, we will set the mobility of receptor dimers lower than that of monomers. These two ingredients result in the preferential accumulation of receptors in the high density patches.

We first identify the best fitting parameters for each file. By comparing the distribution of cluster sizes in that file to what the model gives us, the discrepancy between the number of clusters of each type in the file and the model prediction. We find the 'best' parameter set by varying the parameters and looking for the set that gives the smallest discrepancy. Each file results a different optimal parameter set. If the files are related, the sets are similar. The parameters for a given image as number of receptors $R$ fixed, we estimate number of boxes $N_{B}$, number of high density boxes $N_{B h}$, and the attractiveness of high density region $\alpha$ parameters by minimizing the distance $<\Delta^{2}>$ of a given probability distribution of particles (clustering) on a high density region. Another way comparing our static model with experimental data would be an attempt of 
taking the total number of particles of a given data sets and making a global fit for the entire set of images. Individual fitting file by file, which is not global fits individual files the best but may not be informative about the group. We use this as a preliminary fit, to help identify files that 'belong' together.

The first thing we do is to choose an error function. The most common is the sum of square errors given by

$$
\chi^{2}=\sum_{i} \frac{\left(D\left(t_{i}\right)-y\left(t_{i} \mid \theta\right)\right)^{2}}{2 \sigma^{2}}
$$

where the $\sigma$ is some estimate of the error of the data. We use the experimental uncertainty by making a guess .

We construct a likelihood function which is $P(D \mid \theta)=\exp \left(-\chi^{2}\right)$

Hence, the likelihood function parameter is maximal when the error parameter is minimal. The method of maximum likelihood estimation is simply finding the set of parameters that maximizes the likelihood function.

We use the simple Metropolis algorithm to do a maximum likelihood estimation. We start with some initial guess for the parameter values and compute $\chi_{\text {CurrentFit }}^{2}$. The number of parameters that we like to estimate is more than one parameter so we make a guess for all of them at once. We then propose a new set of parameter values and calculate $\chi_{\text {BestFit }}^{2}$ and compute the likelihood ratio:

$$
\frac{P\left(D \mid \theta_{\text {CurrentFit }}\right)}{P\left(D \mid \theta_{\text {CurrentFit }}\right)}=\exp \left(-\chi_{\text {BestFit }}^{2}+\chi_{\text {CurrentFit }}^{2}\right)
$$

The likelihood ratio is a positive real number. We then pick a uniform random number between 0 and 1 to compare with likelihood ratio. If it is bigger, we replace the current parameter set with the proposed set and then repeat. At each step of the process, we store the value of the current set. It is iterated for 100000 steps and picked the parameter set that had the maximum likelihood.

\section{Is a Global Fit Possible?}

Once we fit the model individually file by file then by using the parameters, we merge all the cluster distribution information for the set of files into one distribution. This is like taking the images and arranging them next to each other, then pretending it is a single larger image. We can then fit a single instance of the model to this joint distribution. One would use the total number of points. As a result it gives a single set of parameters. The global fit, not file by file procedure is a quicker way to get a global fitting parameter set. Individual files may be fit very badly. However, even 
though the procedure is quick, possibly mixing files with too many points (therefore not many small clusters) with files with few points (relatively many small clusters). That information was vague when we merge the distributions. The model assumes random placement of points in the boxes, which is not consistent with having pieces that have widely different densities (i.e. points per file, which in the joint picture become regions). We improve our approach as follows; fitting the model to minimize discrepancy and keeping track of the model by using the same set of parameters simultaneously from individual fits but we also compute a predicted distribution for each file, using its number of points. For each file, we compute the discrepancy, we then construct a joint distance that characterized how well this parameter set works for this group of files. This third way is what we sometimes call global fit file by file. It is global because we seek a single parameter set for the group of files, but it is file by file because we compute a distance (discrepancy) for each file first.

After investigation of fitting the model to data image by image we use the same parameters obtained from individual model fitting file by file for later use. A global fit because it identifies a single parameter set for the entire set of images whereas it is file by file because it computes a discrepancy which is minimized as much as possible for each file first. That allows us to analyze the data not just image by image to perform model fitting globally even though it does not fit each file as well as individual fit file by file but it gives a meaningful characterization of a group of files by assuming the conditions corresponding to those files are similar.

\section{K-Means Clustering}

K-means clustering is generally a good way to to find clusters of comparable spatial extent. The problem of finding the best k-clusters partition among $N$ observation is computationally difficult (NP-hard); however, there are efficient algorithms that converges to a local optimum faster. $\mathrm{K}$-means algorithm uses cluster centers to model the data and the algorithm mechanism allows clusters to have different shapes.

Given a set of observations $\left(x_{1}, x_{2}, \cdots, x_{n}\right)$, k-means clustering aims to partition this $n$ observation into $k \leq n$ sets $C=\left(c_{1}, c_{2}, \cdots, c_{k}\right)$ by minimizing the within-cluster sum of squares. The mathematical definition of finding this k clusters among $\mathrm{n}$ observation is as follows;

$$
\underset{C}{\operatorname{argmin}} \sum_{i=1}^{k} \sum_{x \in C_{i}}\left\|x-\mu_{i}\right\|
$$

To group the somewhat similar images based on the individual model parameters, we use the kmeans clustering for our entire data set. Since we are interested in the distribution of the number of particles per box, to be compared to the distribution of the number of observed particles per cluster, 


\begin{tabular}{|c|c|c|c|c|c|c|}
\hline Filename & $\mathrm{R}$ & \multicolumn{1}{l|}{ Length } & \multicolumn{1}{c|}{ Clusters } & \multicolumn{1}{l|}{ Alpha } & \multicolumn{1}{l|}{$\mathrm{f}=\mathrm{N}$ BH/NBoxes } & $\mathrm{p}^{\wedge} 2 / \mathrm{A} / \mathrm{N} \_\mathrm{C}$ \\
\hline \hline $5-16594$ & 145 & 54.1 & 40 & 119.912 & 0.1245 & 13.0934 \\
\hline $5-16596$ & 14 & 64.57 & 10 & 121.583 & 0.0230 & 12.5660 \\
\hline $5-16598$ & 378 & 47.27 & 37 & 5.24444 & 0.3830 & 14.8046 \\
\hline $5-16598 \mathrm{~b}$ & 179 & 44.65 & 30 & 5.16812 & 0.3667 & 15.0891 \\
\hline $5-16600$ & 24 & 60.17 & 11 & 152.552 & 0.0172 & 12.3885 \\
\hline $5-16600 \mathrm{~b}$ & 42 & 42.63 & 17 & 106.629 & 0.0591 & 12.5477 \\
\hline $5-16602$ & 68 & 41.66 & 31 & 356.649 & 0.0528 & 12.4683 \\
\hline $5-16604$ & 165 & 45.73 & 18 & 8.32877 & 0.2500 & 16.1065 \\
\hline $5-16606$ & 186 & 48.68 & 16 & 95.0296 & 0.0437 & 16.4774 \\
\hline $5-16606 \mathrm{~b}$ & 151 & 54.9 & 12 & 5 & 0.0500 & 13.1122 \\
\hline $5-16608$ & 13 & 43.06 & 7 & 190.504 & 0.0338 & 12.5698 \\
\hline $5-16608 \mathrm{~b}$ & 23 & 53.29 & 7 & 5 & 0.0500 & 12.9514 \\
\hline $5-16610$ & 220 & 41.6 & 23 & 7.66836 & 0.1875 & 15.8192 \\
\hline $5-16610 \mathrm{~b}$ & 217 & 45.93 & 14 & 18.7051 & 0.2500 & 17.6077 \\
\hline $5-16612$ & 21 & 51.04 & 13 & 85.9387 & 0.0431 & 12.8144 \\
\hline $5-16612 \mathrm{~b}$ & 40 & 87.4 & 23 & 363.474 & 0.0585 & 12.5334 \\
\hline $5-16614$ & 30 & 64.34 & 14 & 500 & 0.0234 & 12.6638 \\
\hline $5-16614 \mathrm{~b}$ & 34 & 46.76 & 11 & 121.648 & 0.0179 & 11.6663 \\
\hline $5-16616$ & 70 & 34.63 & 31 & 9.39864 & 0.0667 & 12.4073 \\
\hline $5-16616 \mathrm{~b}$ & 133 & 54.38 & 52 & 4.3895 & 0.2222 & 12.6938 \\
\hline $5-16618$ & 126 & 64.17 & 36 & 3.32178 & 0.3953 & 13.7047 \\
\hline $5-16618 \mathrm{~b}$ & 90 & 52.73 & 36 & 3.77468 & 0.2391 & 12.8368 \\
\hline $5-16620$ & 485 & 55.29 & 150 & 5.77309 & 0.0330 & 11.6834 \\
\hline $5-16620 \mathrm{bkgd}$ & 429 & 57.81 & 195 & 6.18437 & 0.0458 & 12.5699 \\
\hline $5-16622$ & 510 & 77.95 & 192 & 4.03975 & 0.0878 & 12.8593 \\
\hline & & & & & & \\
\hline
\end{tabular}

Table 3: Fitted model parameters: a set of model parameters estimated by Monte Carlo simulation performed on resting cells individually. Column 1 represents the name of individual data, column 2 is the total number of receptor of a corresponding file. The columns are and perimeter represents total perimeter and area of all clusters in an individual data. The rest of the columns are as follows: number of boxes (NB), number of high density boxes (NBH) and the attractiveness $(\alpha)$ are the predictive model parameters. The last column gives the error $\left.\left(<\Delta^{2}\right\rangle\right)$ to compare the experimental data and the model. 
we merge a set of model parameters $\left\{\alpha, N_{B}, f\right\}$ that work for a large set of images, irrespective of the number of receptors captured in the image.

We detect the similar groups in a given data set by performing 6-means clustering. We then again fit those groups into globally file by file and compare the distribution of the number of observed particles per cluster with our model.

\begin{tabular}{|c||r|r||r|r||r|r||r|r||c|}
\hline \multirow{2}{*}{ Cluster } & \multicolumn{2}{c||}{$N_{B}$} & \multicolumn{2}{c||}{$N_{B H}$} & \multicolumn{2}{c||}{$\alpha$} & \multicolumn{2}{c|}{$f(\%)$} & \multirow{2}{*}{ File ct. } \\
\cline { 2 - 8 } & cntrd & \multicolumn{1}{|c|}{ fit } & cntrd & \multicolumn{1}{c|}{ fit } & cntrd & \multicolumn{1}{c|}{ fit } & cntrd & fit & \\
\hline 1 & 41 & 75 & 5.27 & 6 & 8.3 & 10.9 & 11.65 & 8.00 & 15 \\
2 & 1362 & 1093 & 18.38 & 23 & 275.0 & 185.0 & 1.30 & 2.10 & 9 \\
3 & 127 & 100 & 1.68 & 1 & 23.2 & 9.4 & 0.67 & 1.00 & 2 \\
4 & 236 & 335 & 9.59 & 13 & 93.7 & 107.6 & 3.81 & 3.88 & 13 \\
5 & 227 & 266 & 41.07 & 44 & 423.0 & 317.0 & 17.90 & 16.54 & 10 \\
6 & 154 & 134 & 18.05 & 8 & 5.7 & 5.3 & 11.49 & 5.97 & 16 \\
\hline
\end{tabular}

Table 4: Fitted model parameters after we performed k-means clustering for the unknown model parameters for each data set. Columns labelled (cntrd) represent cluster centroids ( $\mathrm{k}=6$, only model parameters). Results of global fitting by k-means group are lebelled (fit).

\subsubsection{Cluster Number Distributions}

At the microscopic level, we investigate the localization of clusters and localization of receptors within the clusters. We test the analysis against electron microscopy images from an experiment in which PAE-KDR cells were exposed for 2-5 minutes to increasing concentrations of antibodies that binds the high affinity VEGF receptor. The cluster scaling curve analysis as shown in Fig.34 confirms the increase in clustering with increasing antibody dose predicted from visual analysis and from Hopkins and Ripley test statistic. A key step to understand the detailed fine scale structure of the cell membrane to access a robust and sensitive tool to both observe and quantify clustering. U1timately, determining the role of spatial organization in the regulation of transmembrane signaling is another step.

Static distributions (see Fig.4), exhibit clusters that are not consistent with a random distribution. The identification of clusters can be done by a hierarchic clustering algorithm. The distribution of nearest neighbor distances, as well as other statistical measures, point toward a structure of high density patches, essentially identified with the observed clusters. Receptors are distributed randomly within the patches, and the patches themselves also appear to be distributed randomly. 

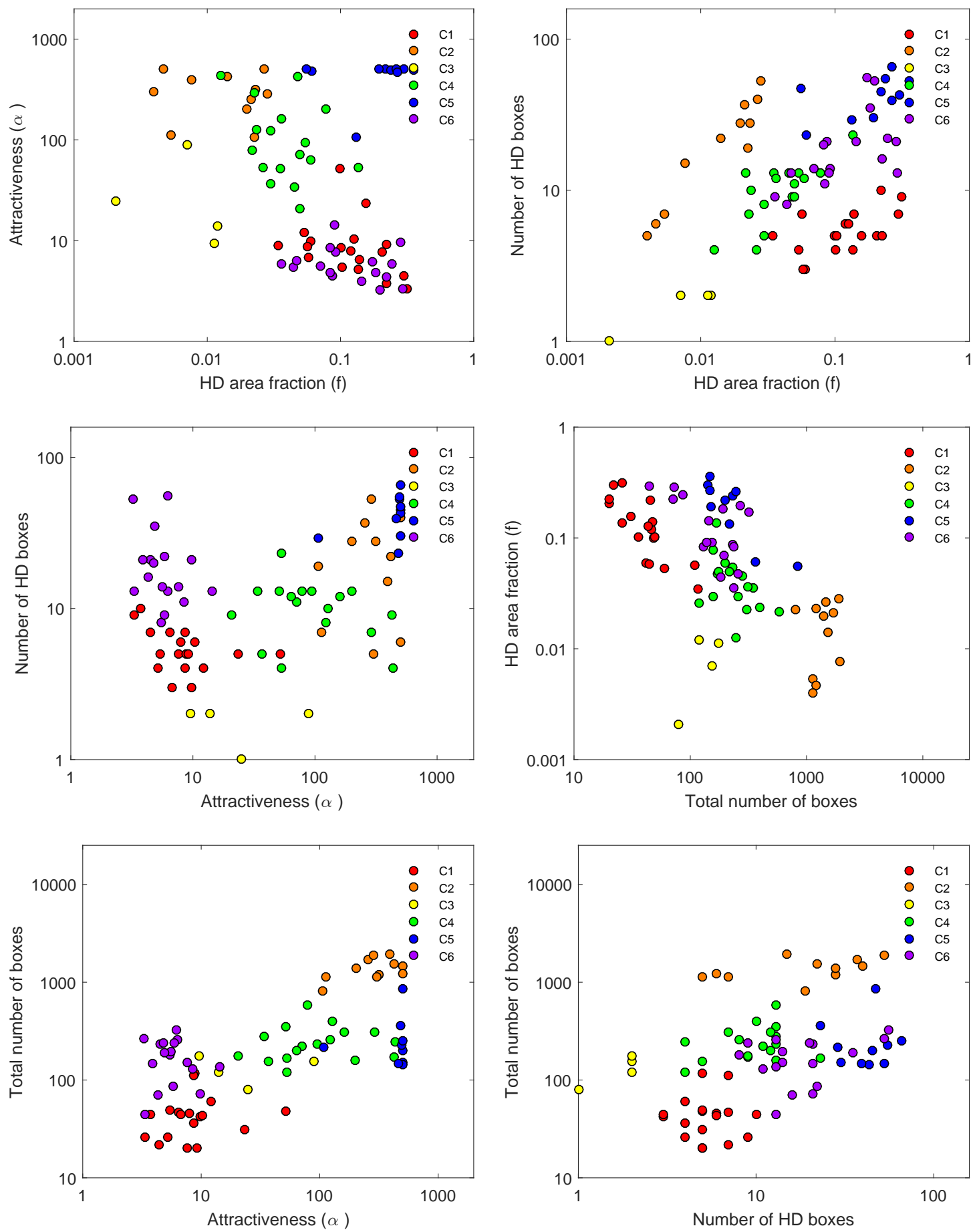

Figure 33: Scatter plots of model parameters after applying 6-means clustering from the individually fitted data. 

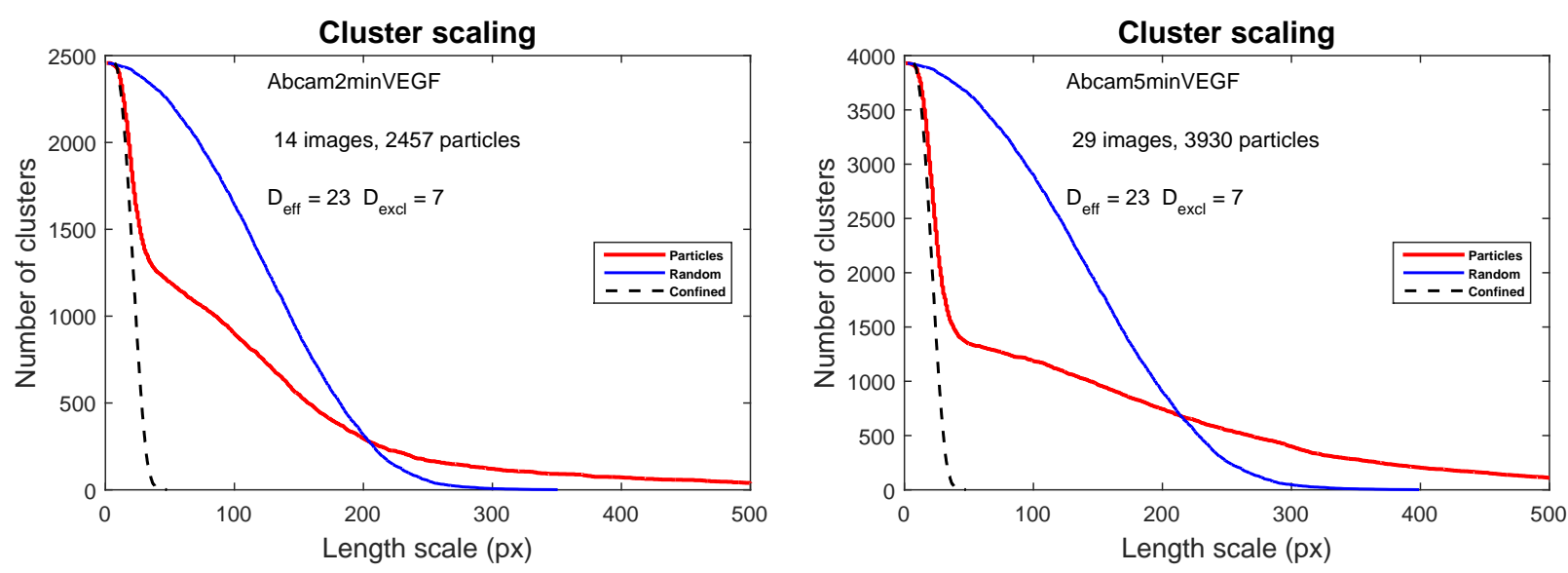

Figure 34: Number of clusters as a function of the length parameter (red lines), and a set of 14 images (left) and 29 images (right), compared to the average number of clusters expected from a random distribution with the same number points in the same area (blue lines). Cumulative distributions of particles plot obtained from Abcam 2 min and 5 min VEGF images.
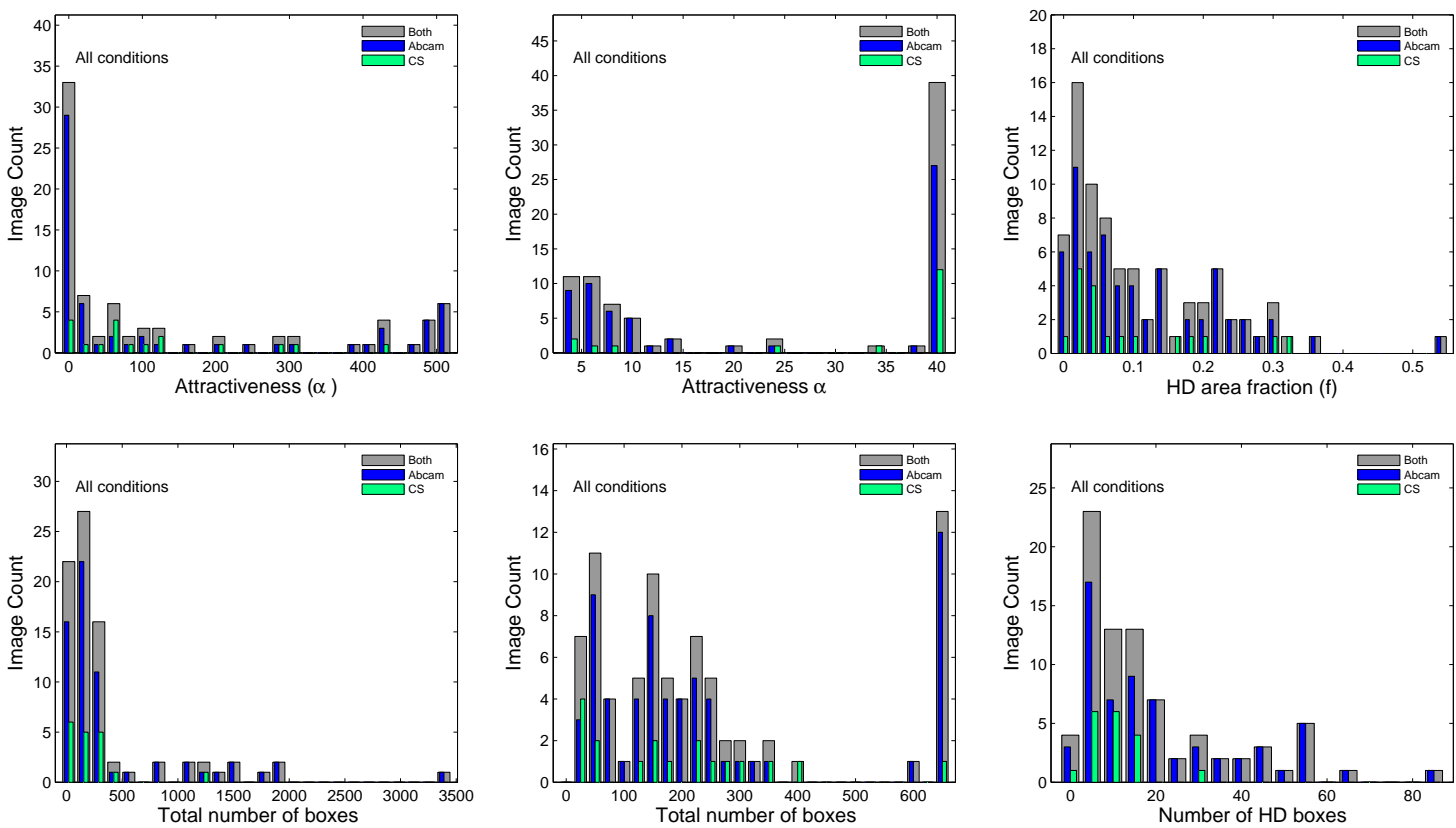

Figure 35: Histograms that demonstrate how model parameters are distributed for all the data sets 

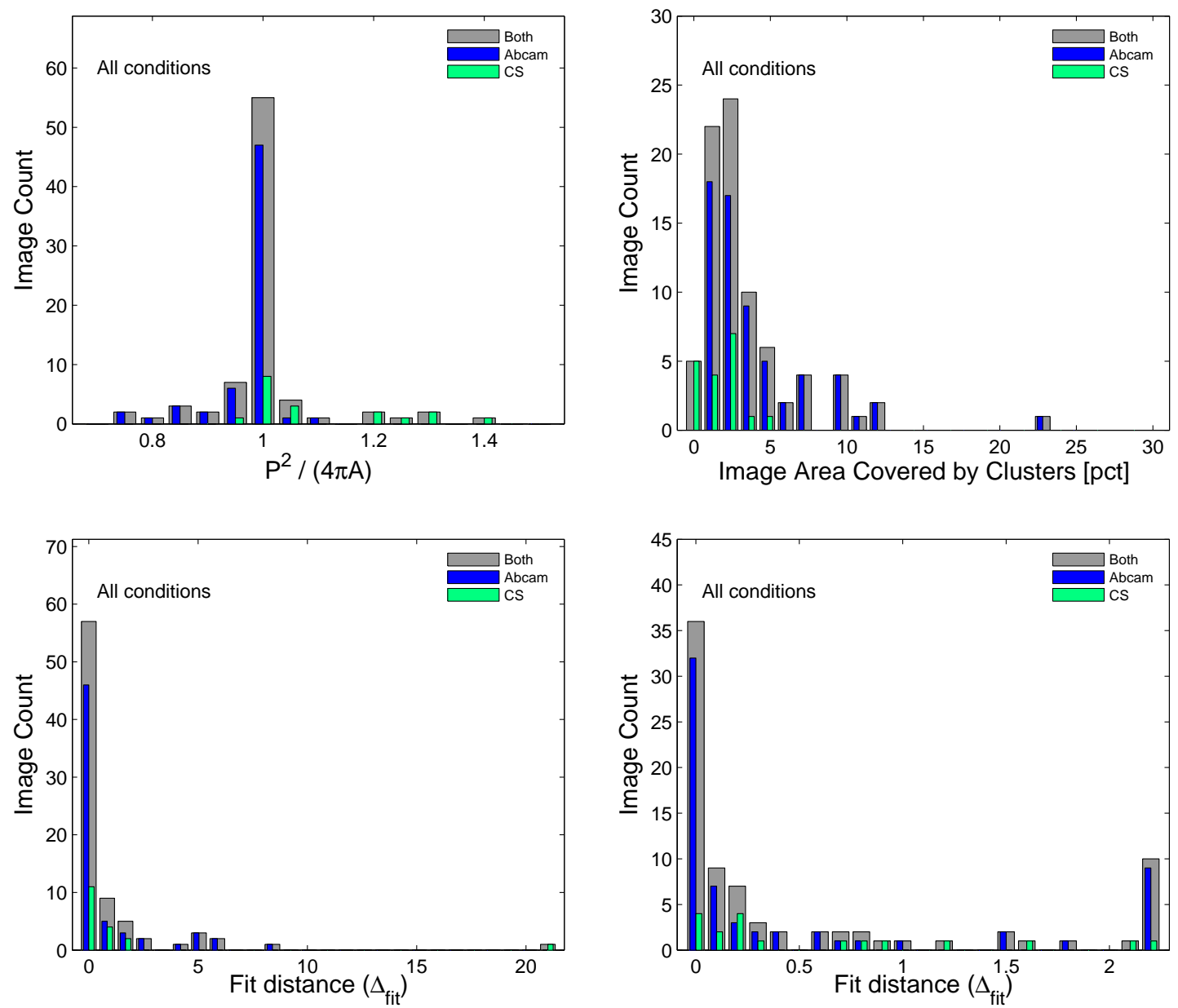

Figure 36: Histograms demonstrates how well our model fits the data 

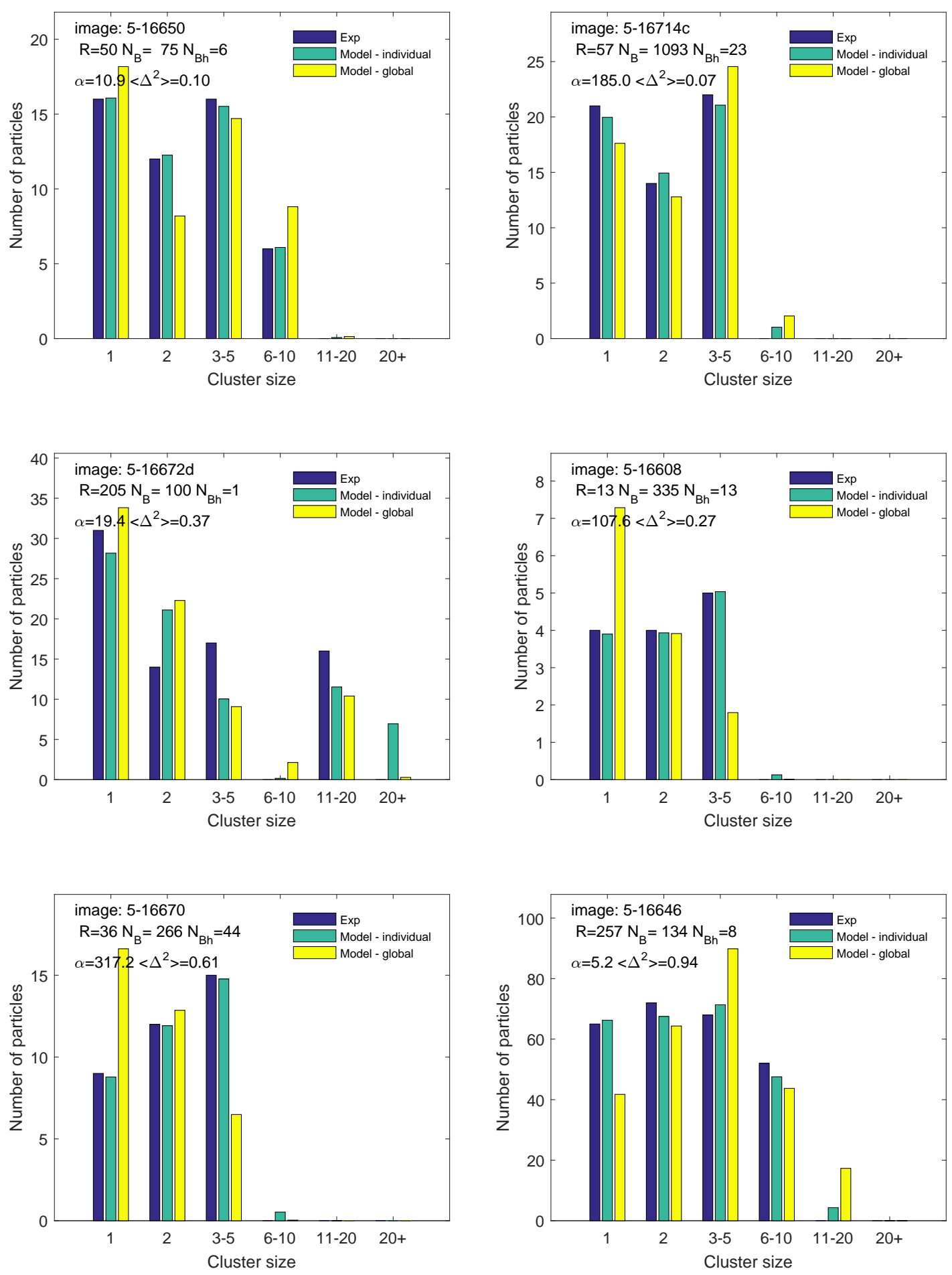

Figure 37: Global fit file by file results after performing 6-means clustering. 

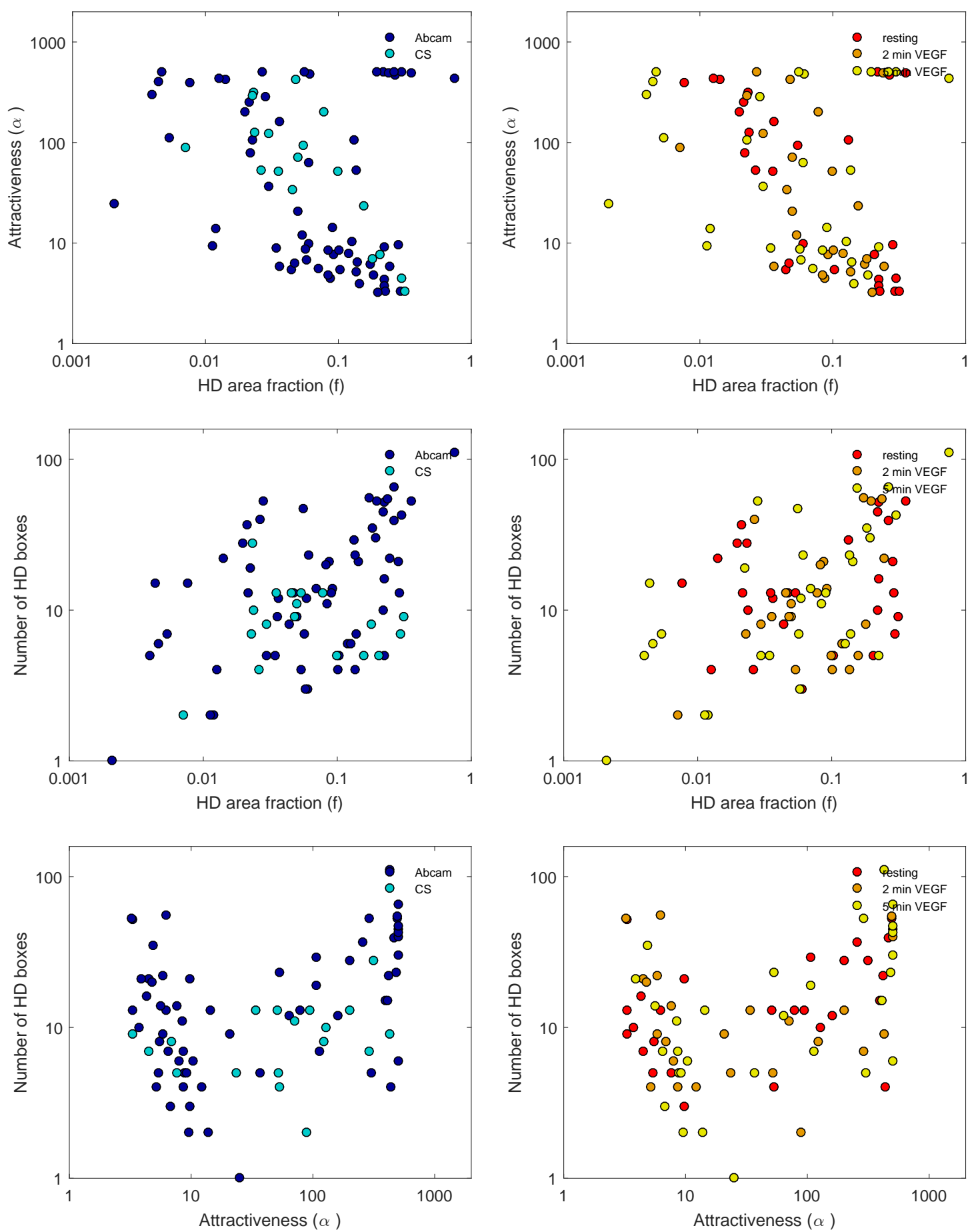

Figure 38: Scatter plots of model parameters for other model parameters. Left: by antibody types, right: by time amount of stimulus 

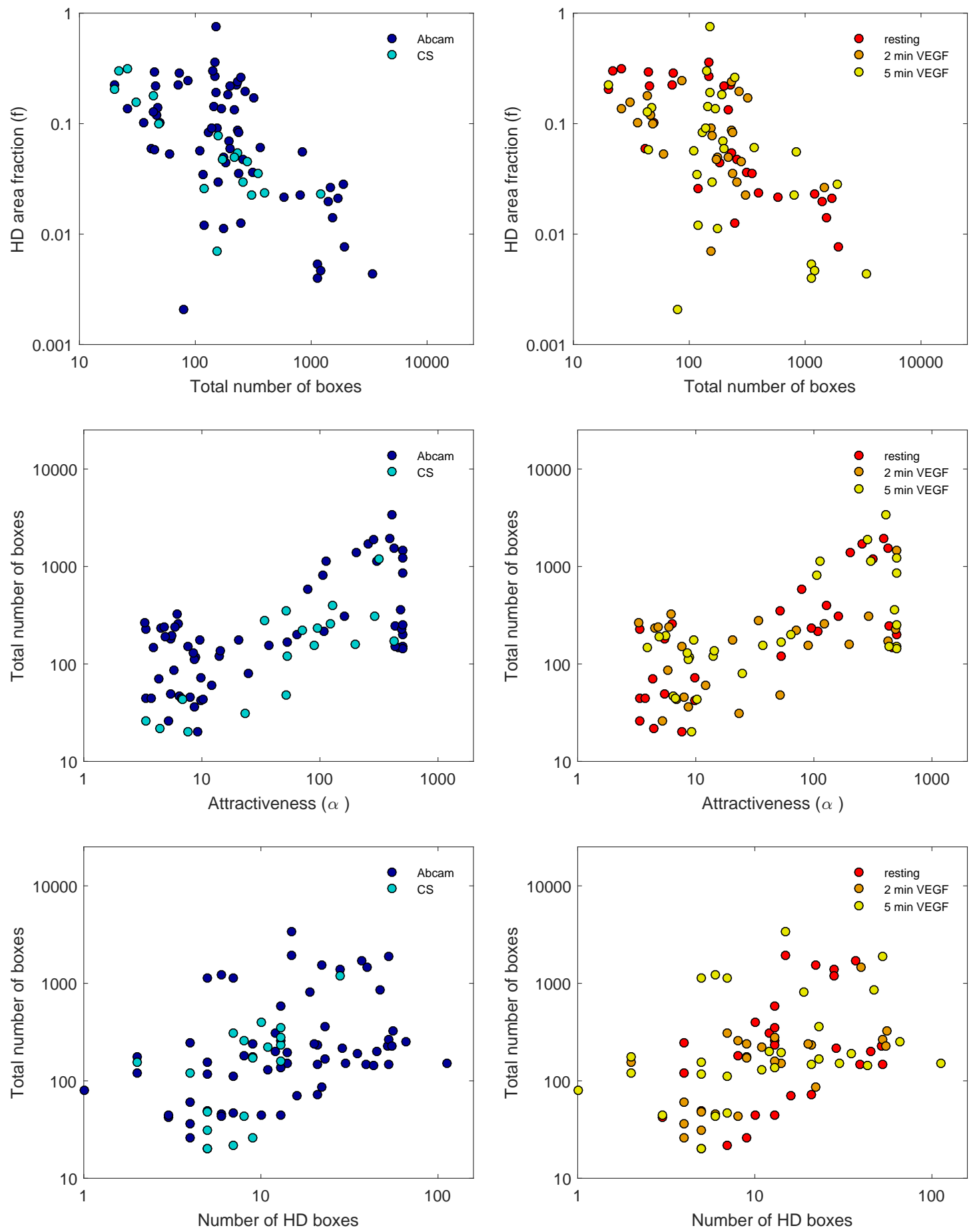

Figure 39: Scatter plots of model parameters for other model parameters. Left: by antibody types, right: by time amount of stimulus 


\section{Chapter 3}

\section{Discussion and Future Work}

Membrane proteins are distributed unevenly and non-randomly on the plasma membranes. Special features of cell membrane subdomains and compartment support the evidence of the heterogeneity. There is strong evidence that these features are lipid rafts, protein islands and cytoskeletal corrals [17]. When cells are stimulated, protein distributions on the cell-membrane change. We analyzed results derived from PAE-KDR cells that were exposed for $2-5$ minutes to increasing concentrations of VEGF, that binds the high affinity VEGF receptor KDR. VEGF receptors were labeled with gold particles for visualization by TEM imaging. The resulting micrographs showed the receptors localized in singletons, small clusters and large clusters. These patterns changed between groups of cells.

We analyzed the distribution of receptors using nearest neighbor distance distributions, Hopkins and Ripley statistic tests [35]. However, the Hopkins and Ripley statistic tests are not enough to quantify the clusters in this type of experiment; they only give a qualitative indication of clustering. Therefore, there is a need of a simple and predictive model which can identify clusters and compare clustering between and within experimental conditions. Previously, Espinoza and co-workers [5] developed an approach based on hierarchical clustering algorithm to define the clustering distance which enables to quantify the density of the clustering in TEM images. Their approach of quantifying the clustering and to compare experimental conditions between activated mast cells with increasing amount of stimulus. In our study, beyond cluster identification, we want to investigate how clusters form. Our hypothesis is that the area of the cell membrane that contains the cluster has special physical properties where receptors tend to collect in attractive micro-domains. After we identify the optimum length to perform the clustering for each image, we implement domain reconstruction algorithm to define shapes of clusters. The shape is defined by a contour around the cluster, following DRA developed previously for the study of live trajectories of labelled receptors [18]. 
After performing the initial statistical analysis of the data, we defined a predictive model, based on the hypothesis of pre-existing micro-domains. We compared the model predictions for the cluster size distributions $g_{k}$ with the experimental data using a least square distance between the model and experimental cluster size distributions, as shown in Fig.37. In the results shown, the fit was performed using a simulated annealing (Metropolis-Hastings) algorithm that quickly identified a range of good fits. The best fit parameters are demonstrated in Fig.37. In this optimization run, we limited $f<0.75$ and $N_{B}>20 . N_{B}$ corresponds to the typical size of a single attractive domain and should therefore be comparable to the typical area occupied by a cluster. Fits of similar optimality can be obtained with significantly different parameter sets, for example $\alpha=4$, $f=31$ and $N_{B}=65$ obtained without imposing the limitations mentioned above. Therefore, this result should not be interpreted as a measurement of these quantities, but rather as a proof of the principle that the observed cluster size distributions are consistent with the high density domain hypothesis. The model parameters can be identified by fitting to the experimentally derived cluster size distributions. Using a Metropolis-Hastings algorithm, we found that the majority of the images (close to 75\%) could be fit individually. The remaining images exhibited large scale features that were not meant to be captured in the model. The global fit of the 60 images with a single model parameter set was less successful. We obtained better results by separating the images into groups using k-means clustering, and then performing global fits to each group taken separately. The biological significance of these emerging groups is not clear at the moment; however, the process yielded sets of parameter values that can readily be used in dynamical calculations as estimates of the quantitative characteristics of the clustering domains. 


\section{APPENDICES}

\section{Appendix A: Implementation}

We provide a complete implementation of the computational methods that has three modules in this project: The initial step of extracting the particle locations from the micrographs was performed using ImageJ, a public domain software package [26] .

Secondly, all the subsequent analysis, mathematical procedures and numerical calculations discussed in thesis were implemented using Matlab. Finally, we store all parameters into a structure in this main script to perform plots and figures where useful for later use. The main script loops through all the coordinate files, perform analyses, and put everything in a structure. We then make plots based on the structure to make the data analysis easier. We save all figures by using SaveThisFigure.m which also automatically format each figure with FormatThisFigure.m that we created as subscripts. By that way we ease the process of reaching the outputs of all saved figures in the related folder.

\section{Appendix B: Statistical Analysis Program Scripts}

\section{Matlab Code}

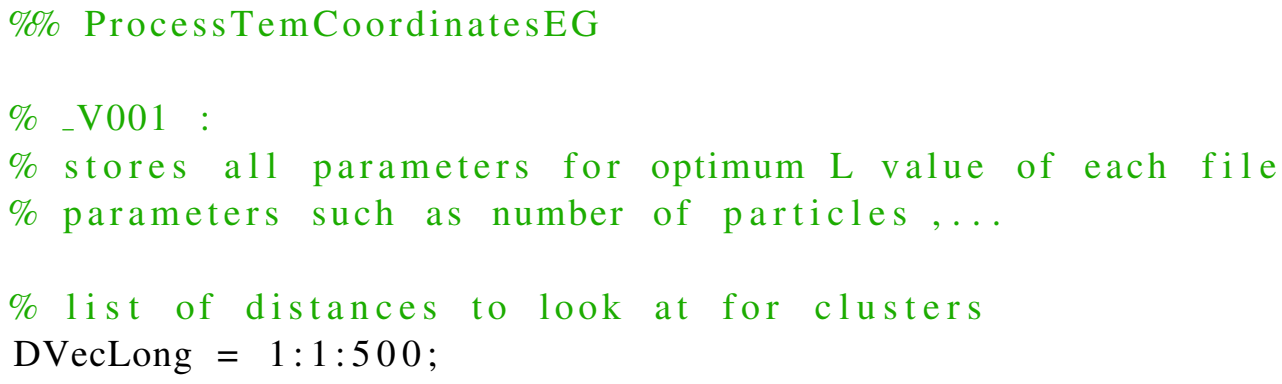




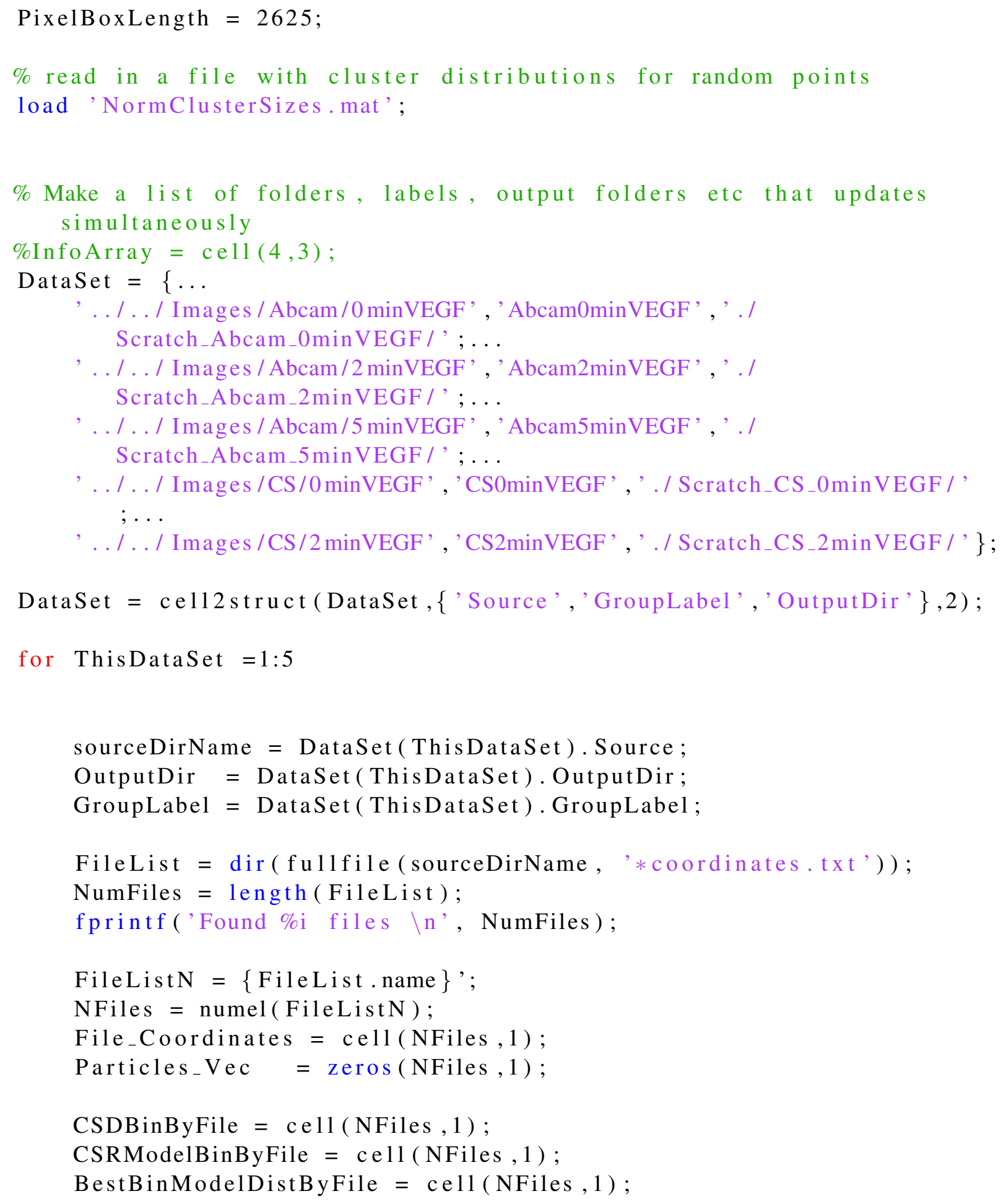


CBoxes = cell (NFiles, 1$) ;$

NBoxesHigh = cell( NFiles, 1$)$;

Alpha $=$ cell $($ NFiles, 1$)$;

ClusterDist $=$ cell $($ NFiles, 1$)$;

\% struct to contain info and data for each file

FileRecord $=\operatorname{struct}(\ldots$

'FileCharacteristics', \{\}$, \ldots$

'BestLInfo', \{\}$, \ldots$

'NearestNeighborDistances' $,\{\}, \ldots$

'Contour', \{\}$, \ldots$

'Hopkins' $,\{\}, \ldots$

'Ripleys' $,\{\}, \ldots$

'ModelParameters', \{\}$)$;

$\%$ FileRecord(NFiles). NParticles $=0$;

MaxParticles $=500$;

CCountRec $=$ zeros $($ NFiles, length $($ DVecLong $)) ; \%$ number of clusters per each file

CSizeRec $=$ zeros (NFiles, length (DVecLong), MaxParticles $) ; \%$ number of

clusters per each file,

$\%$ by length scale and size $1 . .100$

CCountRecCent $=$ zeros $($ NFiles, length $($ DVecLong $)) ; \%$ number of clusters per each file

CSizeRecCent $=$ zeros (NFiles, length (DVecLong), MaxParticles) $; \%$ number of clusters per each file,

$\%$ Hopkins initialzations

HopkinsStatTem=cell (NFiles, 1); \%Hopkins Statistics for each file HopkinsRandStatTem = cell (NFiles, 1$)$;

Hopkins StatCent $=$ cell (NFiles, 1$)$;

HopkinsRandStatCent $=$ cell $($ NFiles, 1$)$;

$\mathrm{dHH}=0.01 ;$

HopkinsHistVec $=0: \mathrm{dHH}: 1$;

\%Ripley's initialzations

RipleysKandLStatTem = cell (NFiles, 1$) ; \%$ Ripleys $\mathrm{K}$ and L Stat

RipleysKandLStatCent $=$ cell (NFiles, 1$)$; 
RValues $=1: 1: 1000$

$\mathrm{NRV}=$ length (RValues $)$;

RipleysTem = zeros $(2, \mathrm{NRV})$;

RipleysCent $=$ zeros $(2, \mathrm{NRV})$;

$\%$ nearest neighbor info

NNDRec $=$ zeros $(1,500) ; \%$ will contain the nearest neighbor distances from all files processed

NND2Rec $=$ zeros $(1,500) ; \%$ next nearest neighbor

NNDRecCent $=$ zeros $(1,500) ; \%$ will contain the nearest neighbor distances for centroids from all files processed

NND2RecCent $=$ zeros $(1,500)$; \%next nearest neighbor distance for centroids

ClusterZ = cell(NFiles, 1$) ; \%$ to collect the clustering results

ClusterCentroidsZ = cell (NFiles, 1$)$; \%to collect the centroids

clustering results

BestL_Vec $=$ zeros (NFiles, 1$) ; \quad \%$ Characteristic L values

BestL_VecCentroids = zeros (NFiles, 1$)$; \% Characteristic L Values for centroids

BestClustersInfo $=$ cell (NFiles, 1$)$;

ClusterLabelsInfo = cell( NFiles, 1$)$;

BestL_ClusterCount $=$ zeros(NFiles, 1$)$; \%corresp. number of clusters OR the number of centroids in each file

BestL_ClusterMap = cell(NFiles, 1$)$; \% cluster membership by point

BestL_Centroids = cell(NFiles, 1$) ; \%$ to colect centroids results for best $\mathrm{L}$

FatContour = cell(NFiles, 1$)$; \% to collect FatContour values for plots

LVec $=1: 1: 500 ; \%$ for cumulative plots to later use

\%Contour Parameters Area and Perimeter Vector

A_Vec $=$ zeros $($ NFiles, 1$)$;

P_Vec $=$ zeros $($ NFiles, 1$)$;

for IFile = 1: NFiles; 


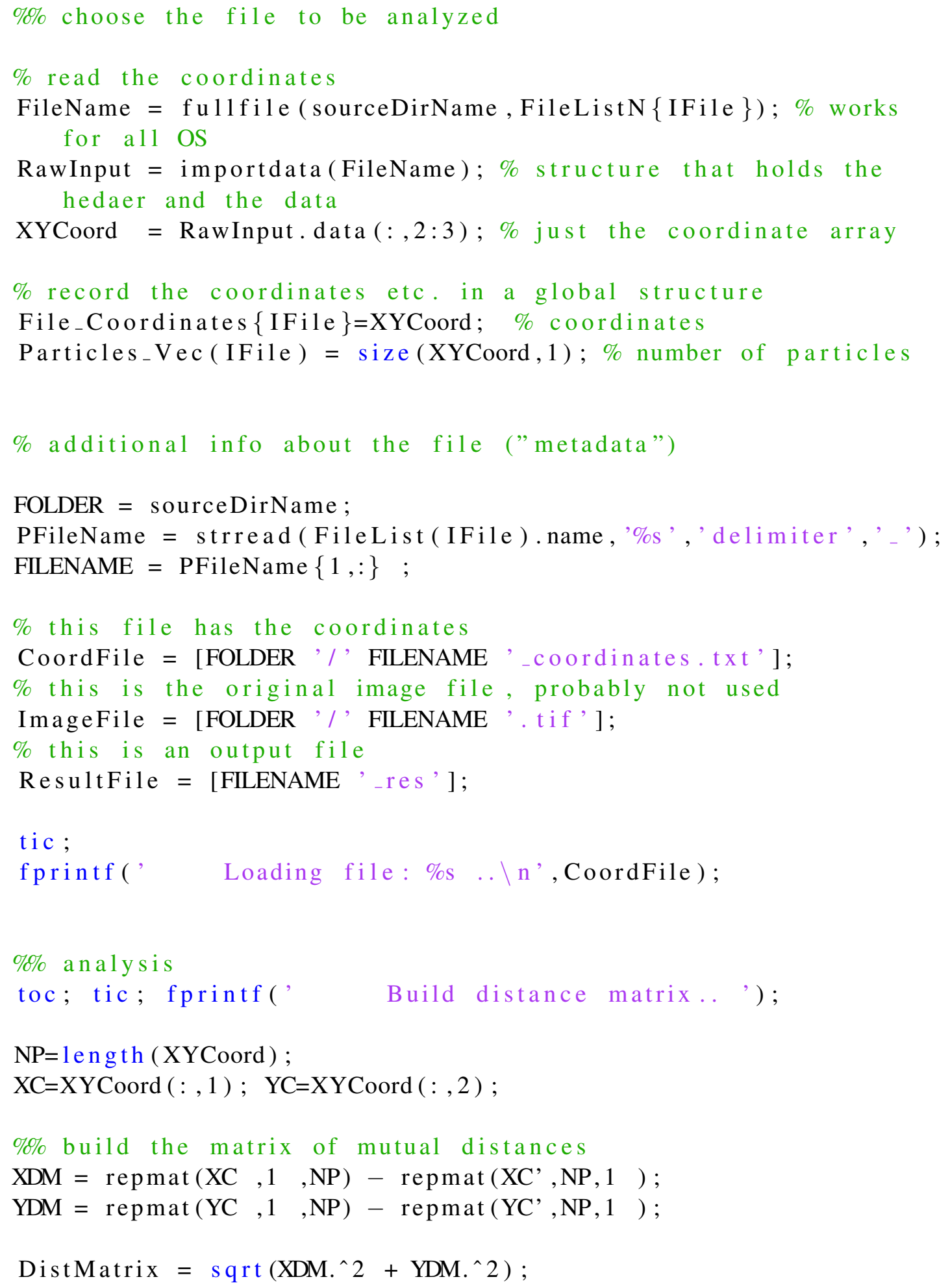




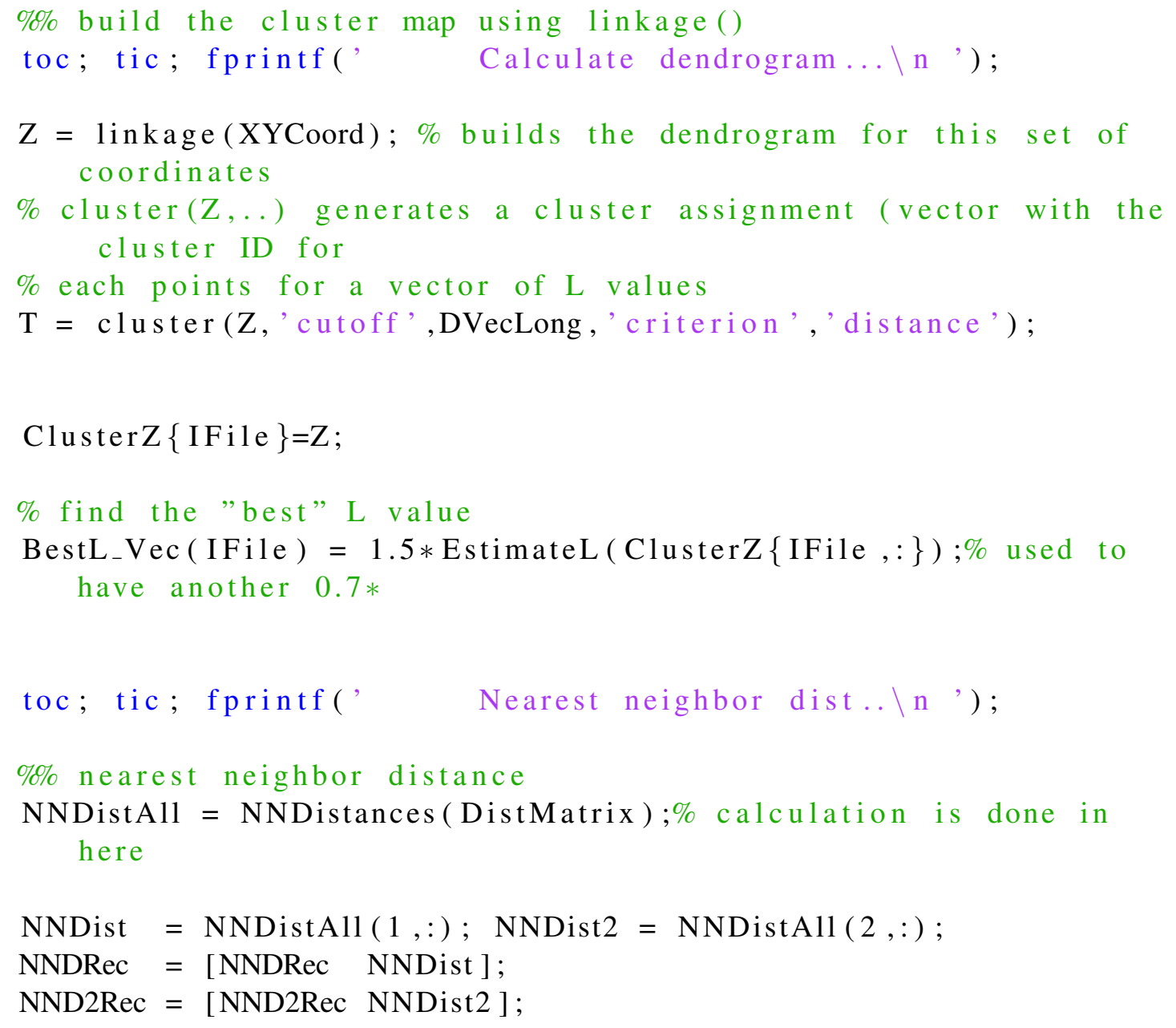


\% cluster, sometimes the cluster we have is only a subset that is ok

\% the suggested length parameter - most of the time all the points form a single

$\mathrm{BL}=\operatorname{sqrt}(\max ($ DistMatrix $))$

$\%$ the factor $1.16=2 / \operatorname{sqrt}(3)$ is to avoid missing "upside down T" mistakes

$\mathrm{BL} 1=\mathrm{BL} * 1.16$

$\%$ FatContour $=$ zeros $(\operatorname{size}($

for icl=1: BestL_ClusterCount(IFile) \% loop over clusters

PointsInCluster = find (BestClusters $==$ ClusterLabels (icl));

ThisClusterPoints = XYCoord(PointsInCluster ,: );

$\%$ Contour calculations

[Contour, Diam $]=$ TightContour $($ ThisClusterPoints, BestL_Vec (

IFile )*1.16);

$\%$ a temporary fix to eliminate inadvertently closed thin contour

if length (Contour) $>1 \& \&$ Contour (end) $==$ Contour $(1)$, Contour $=$ Contour (1: end -1$)$; end ;

$\%$ close the tight contour

$\mathrm{CC}=$ [Contour' Contour( 1$)] ; \%$ this is a set of indices

XYContourClosed $=$ ThisClusterPoints $(\mathrm{CC},:) ; \%$ this is a NPC $\mathrm{x}$ 2 array of coordinates

$\%$ give the tight contour (the OPEN version) to the fat

contour function

XYContour $=$ ThisClusterPoints $($ Contour,$:)$;

$\%$ FC is the (closed) fat contour as a NPC $x 2$ array of coordinates

$\mathrm{FC}=$ FatContourBuild (XYContour, BestL_Vec(IFile ), pi/12);

$\% c a l c u l a t e$ the area of cluster

A_Vec (IFile $)=$ A_Vec $($ IFile $)+$ poly area $(F C(:, 1), F C(:, 2))$;

if isnan(A_Vec(IFile)), return; end;

$\%$ calculate the perimeter of cluster

$\mathrm{DFC}=\mathrm{FC}(2:$ end,$:)-\mathrm{FC}(1:$ end $-1,:) ;$

P_Vec $($ IFile $)=P_{-}$Vec $($IFile $)+\operatorname{sum}(\operatorname{sqrt}(\operatorname{sum}($ DFC.^2,2) $))$;

MyClusterMap (PointsInCluster) = icl;

MyCentroids (icl,$:)=$ mean $($ XYCoord $($ PointsInCluster , : ) , 1); 
end

BestL_ClusterMap $\{$ IFile $\}=$ MyClusterMap;

BestL_Centroids $\{$ IFile $\}=$ MyCentroids;

BestClustersInfo $\{$ IFile $\}=$ BestClusters;

ClusterLabelsInfo $\{$ IFile $\}=$ ClusterLabels;

$\%$ FatContour $\{$ IFile $\}=$ FC;

$\%$ analysis

toc; tic; fprintf(' Build distance matrix for centroids .. $\left.\backslash \mathrm{n}^{\prime}\right)$;

$\mathrm{NC}=$ length ( BestL_Centroids $\{$ IFile $\})$;

CXCoord=BestL_Centroids $\{$ IFile $\}(:, 1)$; CYCoord=BestL_Centroids \{ IFile $\}(:, 2)$;

$\% \%$ build the matrix of mutual distances

$\mathrm{CXDM}=\operatorname{repmat}(\mathrm{CXCoord}, 1, \mathrm{NC})-\operatorname{repmat}\left(\mathrm{CXCoord}^{\prime}, \mathrm{NC}, 1\right)$;

$\mathrm{CYDM}=\operatorname{repmat}(\mathrm{CYCoord}, 1, \mathrm{NC})-\operatorname{repmat}\left(\mathrm{CYCoord}^{\prime}, \mathrm{NC}, 1\right)$;

DistMatrixCent $=\operatorname{sqrt}\left(\mathrm{CXDM} \cdot \cdot^{\wedge} 2+\mathrm{CYDM}^{\wedge}{ }^{\wedge} 2\right)$

$\%$ \%bulid the cluster map of centroids using linkage() again

toc; tic; fprintf(' Calculate dendrogram for centroids.. \n');

ZCentroids $=$ linkage $($ BestL_Centroids $\{$ IFile $\}) ;$

ClusterCentroidsZ $\{$ IFile $\}=$ ZCentroids;

$\%$ find the "best" L value

$\%$ set it to 500 if $>500$

BestL_VecCentroids (IFile) $=0.7 *$ EstimateL (ClusterCentroidsZ \{ IFile ,: \}) ;

$\%$ record for cumulative analysis

$\%$ CCountRec (IFile, $:$ ) = BestClusters;

$\% \%$ calculate the number of clusters for each length value in DVecLong

ClusterCount $=\operatorname{zeros}(\operatorname{size}($ DVecLong $)) ;$

$\%$ the cluster goes down when $L$ is equal to the split distance

$\%$ (i.e. the entries of Z) 


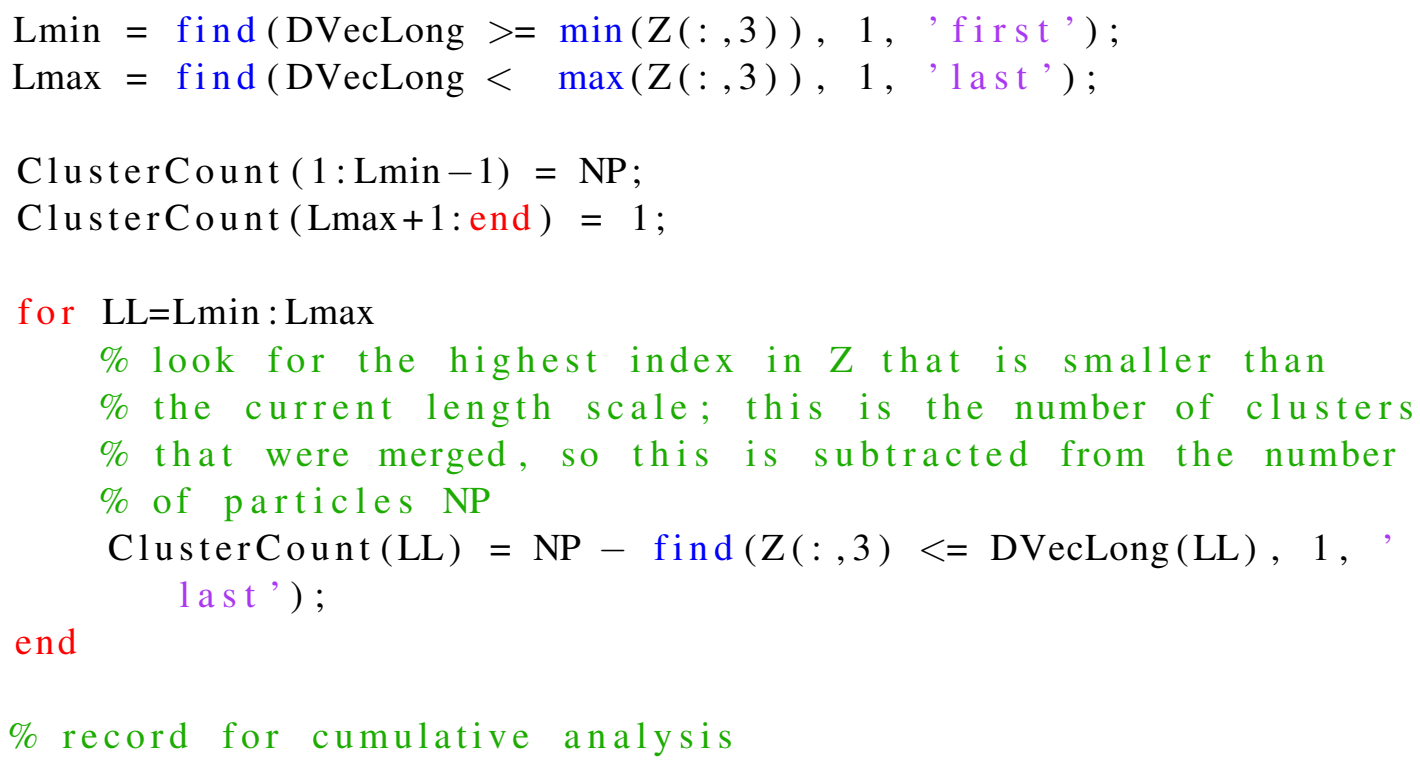

toc; tic; fprintf(' Hopkins and Ripleys stats for particles.. $\backslash n$ ) ;

\%\% Particles Hopkins and Ripleys Stat

\%Hopkins Test Initialzations

$[\mathrm{m} 1, \mathrm{n} 1]=\operatorname{size}(\mathrm{XYCoord})$;

HopkinsStatTem $\{$ IFile $\}=$ HopkinsT (XYCoord, 1, 1,3);

HopkinsRandStatTem $\{$ IFile $\}=$ HopkinsT $(\operatorname{rand}(\mathrm{m} 1, \mathrm{n} 1), 1,1,3)$; 


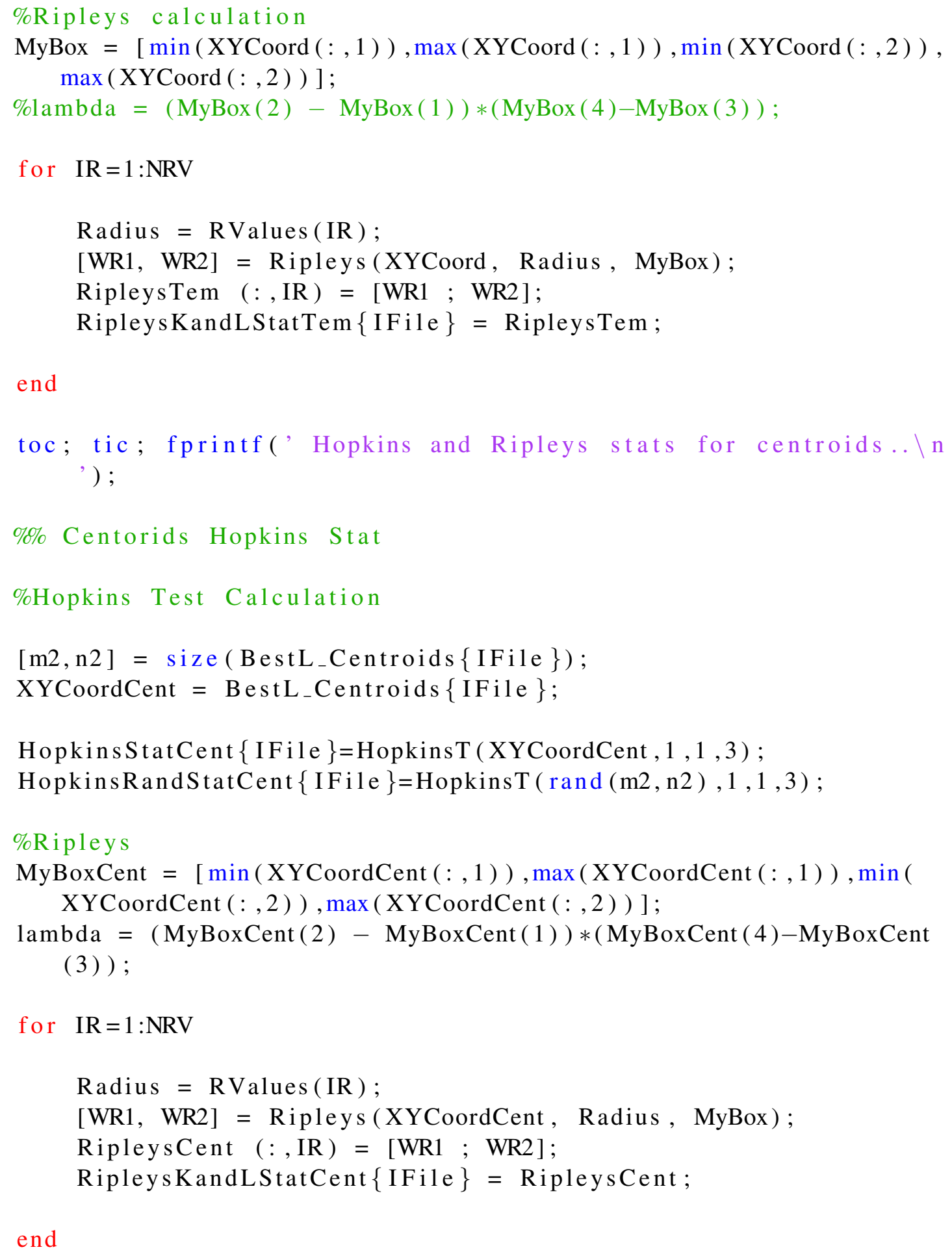


toc ;

BestLength=BestL_Vec (IFile , 1);

$\mathrm{IL}=$ find $($ DVecLong $==$ floor $($ BestLength $), 1)$

ClusterList $=$ zeros $(\mathrm{NP}, 3)$;

NClusters = length (ClusterLabels);

CCount $($ floor $($ BestLength $))=$ NClusters ;

ClusterList $(1:$ NClusters, 1$)=$ ClusterLabels; $\%$ original label Clusters $=\mathrm{T}(:, \mathrm{IL})$;

ClusterRL = ClusterList (Clusters, 3$) ; \%$ points relabelled

for $\mathrm{IC}=1: \mathrm{NClusters}$

ClusterList $($ IC, 2$)=\operatorname{sum}($ Clusters $==\mathrm{ClusterList}(\mathrm{IC}, 1)) ; \%$ size

ClusterList $(\mathrm{IC}, 3)=$ find $(\mathrm{Clusters}==\mathrm{ClusterList}(\mathrm{IC}, 1), 1) ; \%$ new 1 abel

end

$\mathrm{HC}=\mathrm{h}$ istc $(\mathrm{ClusterList}(1:$ NClusters ,2), $1:$ MaxParticles $)$;

$\% \quad$ CSizeRecCumulative = zeros (NFiles, length (DVecLong), 100$)$;

$\% \quad$ CSizeRecCumulative (: , find (DVecLong==floor (BestLength), 1)

$, \therefore)=\operatorname{HC}(1:$ MaxParticles $) . *(1:$ MaxParticles )';

CSizeRec(IFile, find (DVecLong==floor (BestLength), 1), :) $=\mathrm{HC}(1$ :

MaxParticles ).*( 1 : MaxParticles )';

$\%$ pull out the cluster distribution for BestL \#IL

$\mathrm{CSD}=$ squeeze $($ CSizeRec $($ IFile, IL,$:))$;

MaxCluster $=30$;

$\operatorname{CSD}=\operatorname{CSD}(1: \text { MaxCluster })^{\prime} ; \%$ we are only looking at one file here

CBins $=\left[\begin{array}{llllll}1 & 2 & 5 & 10 & 20 & 200\end{array}\right]$;

NBins $=$ length $($ CBins $)$;

BinProjector $=$ zeros (MaxCluster, NBins $)$;

for $\mathrm{IBin}=1: \mathrm{NBins}$

$\%$ column IBin of BinProjector has 1 at rows that go into this bin

$\%$ and zero otherwise 
if $\operatorname{IBin}==1, \quad \mathrm{CMin}=1 ;$ else $\operatorname{CMin}=\operatorname{CBins}(\operatorname{IBin}-1)+1$; end

if $\operatorname{IBin}==1$ ength (CBins), CMax $=\min (\mathrm{CBins}($ end), MaxCluster $)$; else CMax=CBins (IBin); end;

\% fprintf('Bin \%d: [\%d \%d] $\backslash \mathrm{n}$ ', IBin, CMin, CMax);

BinProjector $($ CMin: $\mathrm{CMax}, \mathrm{IB}$ in $)=1$;

end

\% Particle per cluster size counts by bin

CSDBin $=$ CSD $*$ BinProjector

$\%$ B. Fitting

$\%$ B.1. Initialize (sample model parameters)

NBoxes $=500$;

$\mathrm{f}=0.05$;

alpha $=5.0$

$\mathrm{NBH}=$ ceil $(\mathrm{f} *$ NBoxes $)$;

$\%$ model distribution to compare with

$\% \quad$ \#points, \#boxes, alpha, f

$\% \mathrm{~S}=\operatorname{sum}(\mathrm{A}, \operatorname{dim})$ returns the sum along dimension dim.

$\%$ For example, if $\mathrm{A}$ is a matrix, then $\operatorname{sum}(\mathrm{A}, 2)$ is a column vector

\%containing the sum of each row

NPoints $=\operatorname{sum}($ CSDBin $) ;$

$\%$ model prediction of the cluster distribution

$\%$ using the parameter set [NBoxes, NBH, alpha]

CSDModel $=$ HDClusterDist $($ NPoints, NBoxes, NBH, alpha);

if NPoints $<$ MaxCluster,

$\%$ pad the model output if necessary

CSDModel $=[$ CSDModel zeros $(1$, MaxCluster - NPoints $)]$; else

$\%$ cut off the model at MaxCluster

CSDModel $=$ CSDModel $(2:$ MaxCluster +1$) *$ NPoints $/ \operatorname{sum}($

CSDModel (2: MaxCluster +1$))$;

end ;

\% ready to compare with the data

CSRModelBin $=$ CSDModel $(1: 30) *$ BinProjector ; 


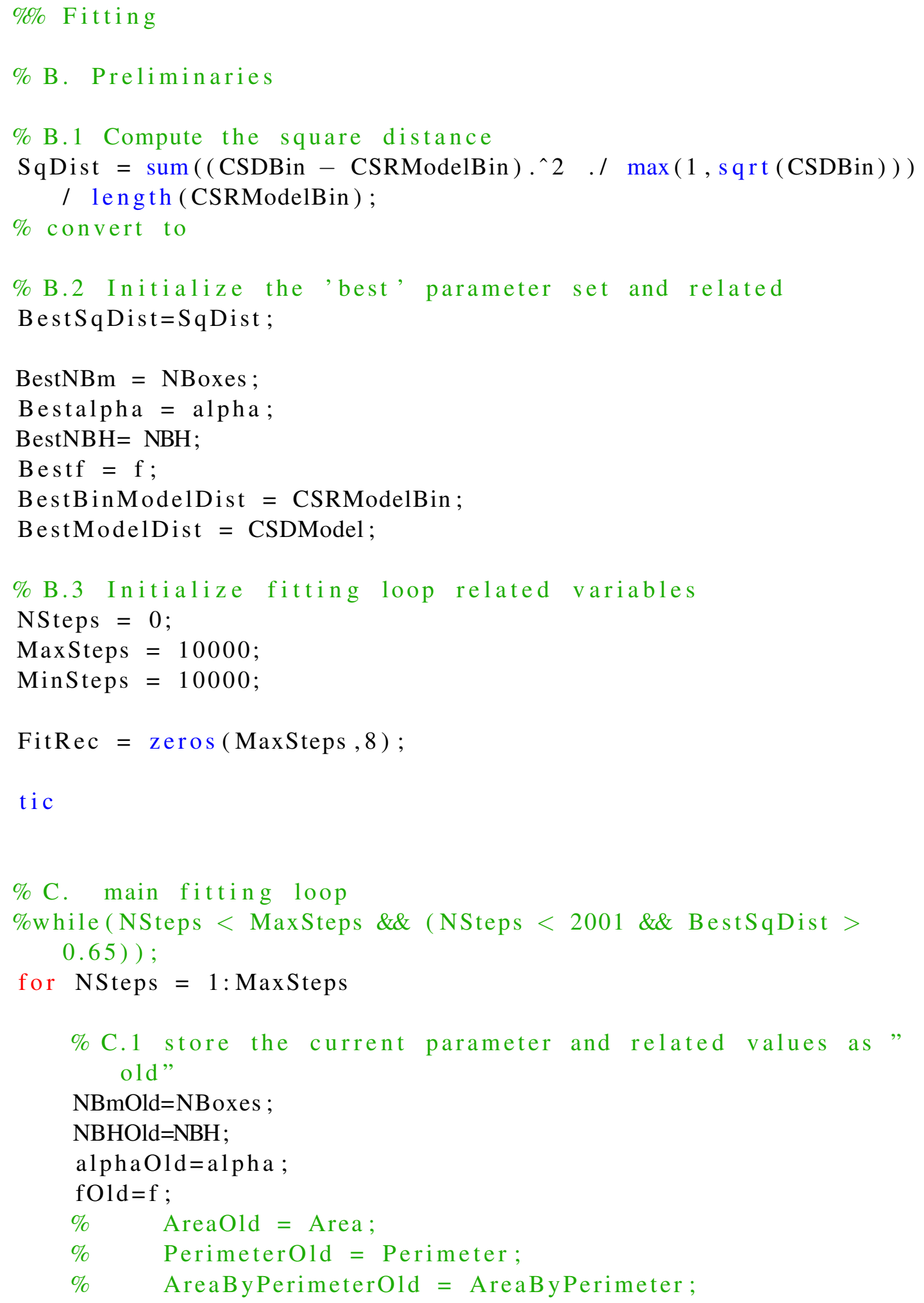




\section{OldSqDist $=$ SqDist}

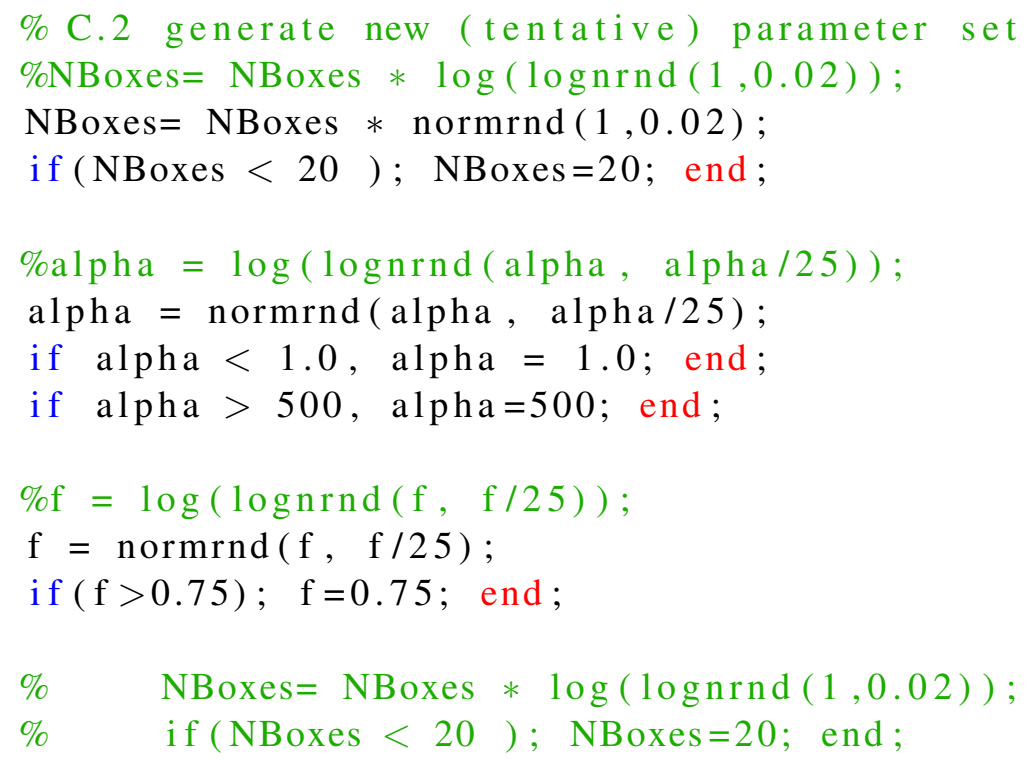

\% C.3 Model prediction using the tentative parameters

CSDModel $=$ HDClusterDist $($ NPoints, NBoxes, NBH, alpha); if NPoints $<$ MaxCluster,

$\%$ pad the model if necessary

$\%$ CSDModel $=[$ CSDModel zeros $(1$, MaxCluster - NPoints $)]$;

$\%$ THIS NEEDS TO BE CHECKED \%

CSDModel $=[$ CSDModel $(2$ : end $)$ zeros $(1$, MaxCluster NPoints ) ];

else

$\%$ cut off the model at MaxCluster

CSDModel $=$ CSDModel $(2:$ MaxCluster +1$) *$ NPoints $/ \operatorname{sum}($ CSDModel (2: MaxCluster +1));

end ;

CSRModelBin $=$ CSDModel $(1: 30) *$ BinProjector 


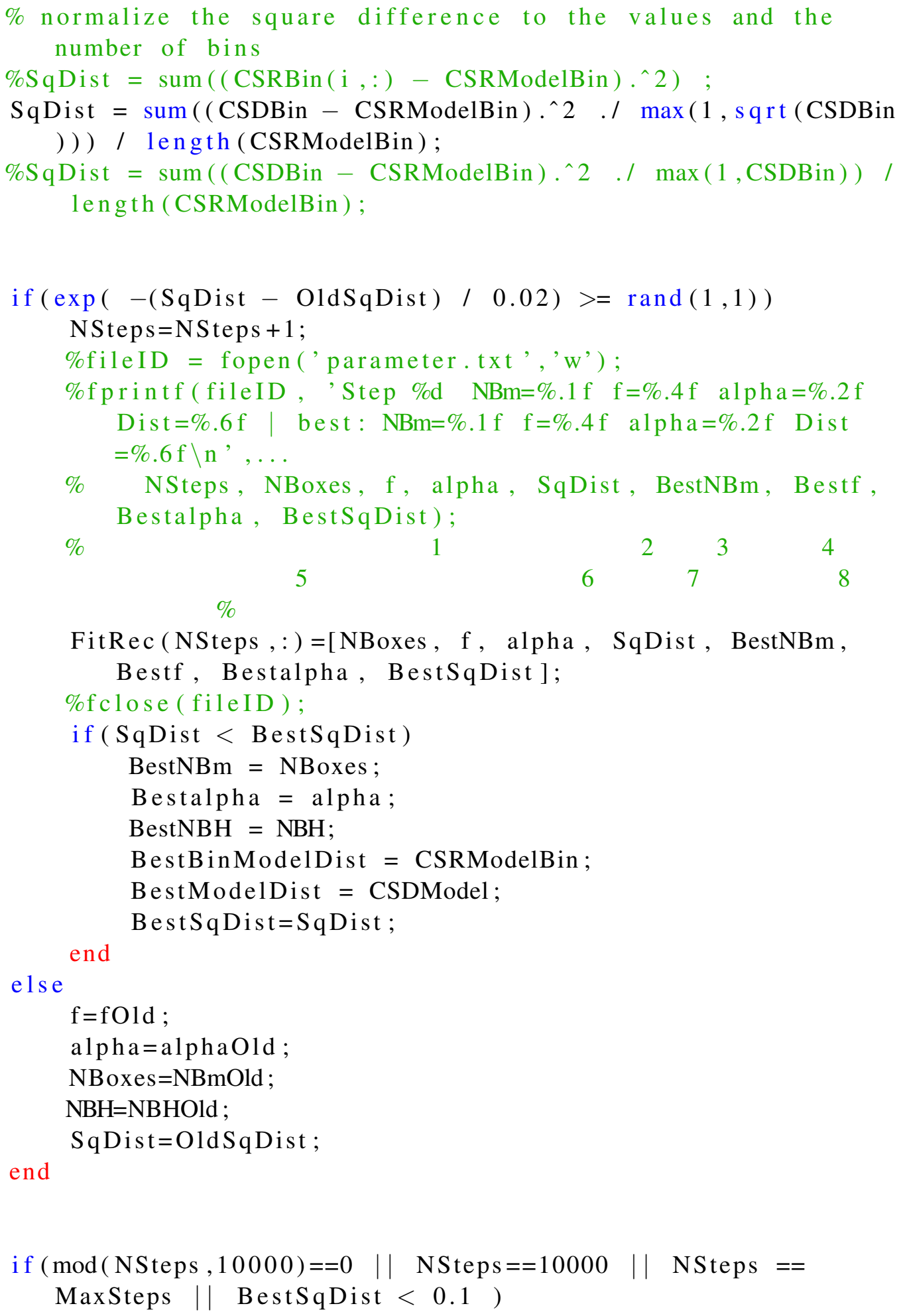


$\mathrm{z}=\mathrm{BestSqDist}$;

$\mathrm{z}=1-(\operatorname{erf}(\mathrm{z} / \mathrm{sqrt}(2))-\operatorname{erf}(-\mathrm{z} / \mathrm{sqrt}(2))) / 2$;

end

$\%$ even stop it at 1.0

if BestSqDist $<0.1$, break; end;

end

toc

CSDBinByFile $\{$ IFile $\}=$ CSDBin;

CSRModelBinByFile $\{$ IFile $\}=$ CSRModelBin;

BestBinModelDistByFile $\{$ IFile $\}=$ BestBinModelDist ;

CBoxes $\{$ IFile $\}=[$ NBoxes, BestNBm]; \% number of boxes recorded NBoxesHigh $\{$ IFile $\}=[\mathrm{NBH}$, BestNBH $]$;

Alpha $\{$ IFile $\}=[$ alpha, Bestalpha $]$;

ClusterDist $\{$ IFile $\}=[$ BestSqDist, $\mathrm{z}]$;

\%or cumulative model fitting

$\mathrm{CSR}=$ squeeze $(\mathrm{CSizeRec}(:, \mathrm{IL},:))$;

$\%$ put this into the new structure

$\%$ Record all Clustering info related to data charactersitics

$\%$ Look into FileRecord. BestLInfo

FileRecord(IFile). FileCharacteristics = struct('FileName', FILENAME, . . .

'FileInfo', GroupLabel ,...

'NImages', NFiles ,...

'XYCoord', XYCoord , . .

'NParticles', size (XYCoord, 1$), \ldots$

'NParticles_Vec', Particles_Vec);

$\%$ Record all Clustering info related to Best $L$ and Centroids

$\%$ Look into FileRecord. BestLInfo 
FileRecord (IFile). BestLInfo = struct('BestL', BestL_Vec(IFile), 'BestLVector', BestL_Vec ,...

'ClusterZ',Z, ...

'BestLClusterCount ', BestL_ClusterCount (IFile ) , BestLCC_Vec' , BestL_ClusterCount ,...

'BestLCentroids', BestL_VecCentroids (IFile ), ...

'ClusterZCentroids', ZCentroids,...

'ClusterDistBestL', CSD, ...

'CumulativeClusterDistBestL', CSR, ...

'CSizeRecCumulative', CSizeRec, ...

'CumulativeClusterCount', CCountRec);

$\%$ Record all NearestNeighborDistances

\% Look into FileRecord. NearestNeighborDistances

FileRecord(IFile). NearestNeighborDistances = struct ('

DistanceMatrixParticles ', DistMatrix , 'NNDParticles ',NNDRec ,'

NND2Particles' , NND2Rec, ...

'DistanceMatrixCentroids ', DistMatrixCent, 'NNDCentroids', , NNDRecCent, ' NND2Centroids ', NND2RecCent) ;

\%Record all Contour information for Best L

$\%$ Look into FileRecord. Contour

FileRecord (IFile). Contour = struct ('FatContour' $, F C, \ldots$

'ThisBestClusters', BestClustersInfo\{IFile $\}, \ldots$

'ThisClusterLabels', ClusterLabelsInfo $\{$ IFile $\}, \ldots$

'BestLClusterCount', BestL_ClusterCount (IFile), ...

'Area', A_Vec(IFile), ' Perimeter', P_Vec(IFile) ,...

'AreaVec',A_Vec, 'PerimeterVec', P_Vec);

\%Record all Hopkins Statistic Test parameters

\%Look into FileRecord. Hopkins

FileRecord(IFile). Hopkins = struct ('Particles', HopkinsStatTem(

IFile ), 'ParticlesRandom ', HopkinsRandStatTem (IFile ),...

'Centroids', HopkinsStatCent (IFile), 'CentroidsRandom', HopkinsRandStatCent ( IFile));

\%Record all Ripleys Statistic Test parameters

\%Look into FileRecord. Ripleys structure

FileRecord (IFile). Ripleys = struct ('Particles',

RipleysKandLStatTem $\{$ IFile $\}, \ldots$

'Centroids', RipleysKandLStatCent $\{$ IFile $\}$ ); 


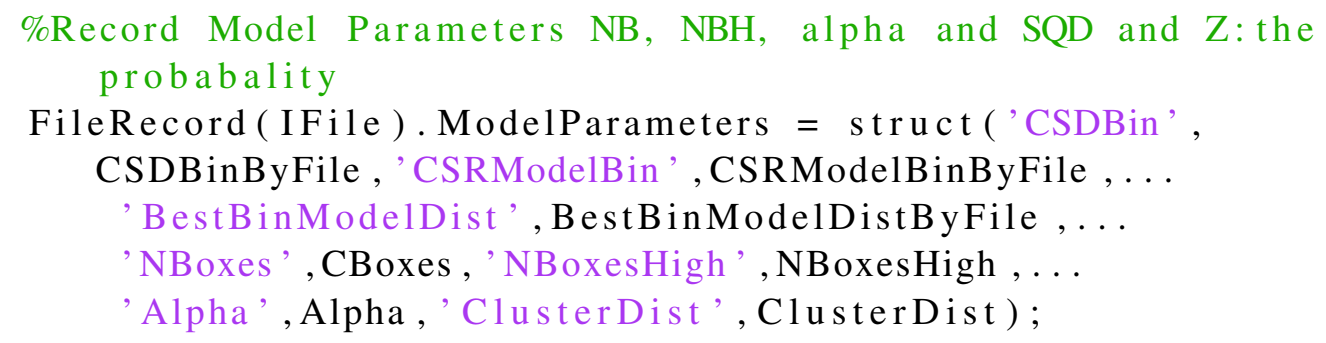

end

\section{Appendix C: Creating Figures from Structure}

We loop through and analyze all the data with main script (see Appendix B) which stores all parameters in a structure. We then use that information and perform plots based on the relevant structure. 


\section{Bibliography}

[1] E. M. Bublil and Y. Yarden. The egf receptor family:spearheading a merger of signaling and therapeutics. Curr. Opin. Cell Biol., 19(2):124-134, 2007.

[2] O. Casanovas, D. J. Hicklin, G. Bergers, and D. Hanahan. Drug resistance by evasion of antiangiogenic targeting of vegf signaling in late-stage pancreatic islet tumors. Cancer cell, 8(4):299-309, 2005.

[3] Y. Chen, C. Short, Á. M. Halász, and J. S. Edwards. The impact of high density receptor clusters on VEGF signaling. Electronic Proceedings in Theoretical Computer Science, pages 37-52, September 2013.

[4] M. Edidin. Lipid microdomains in cell surface membranes. Curr Opin Cell Biol, 7(4):528532, 1997.

[5] F. A. Espinoza, J. M. Oliver, B. S. Wilson, and S. L. Steinberg. Using Hierarchical Clustering and Dendrograms to Quantify the Clustering of Membrane Proteins. Bull. Math. Biol., 74(1):190-211, 2011.

[6] B. B. Friday and A. A. Adjei. Advances in targeting the ras/raf/mek/erk mitogen-activated protein kinase cascade with mek inhibitors for cancer therapy. Clin. Cancer Res., 14(2):342346, 2008.

[7] R. A. Govindan. Review of epidermal growth factor receptor/her2 inhibitors in the treatment of patients with non-small-cell lung cancer. Clin. Lung Cancer, 11(1):8-12, 2010.

[8] D. Hanahan and J. Folkman. Patterns and emerging mechanisms of the angiogenetic switch during tumorigenesis. Cell, 86:353-364, 1996.

[9] A.K Jain, M.N. Murty, and P.J. Flynn. Data Clustering: A Review. ACM Computing Surveys, 31(3):264-323, September 1999.

[10] J. P. Klooster and P. L. Hordijk. Targeting and localized signalling by small gtpases. Biol. Cell, 99(1):1-12, 2007. 
[11] A. Kusumi, C. Nakada, K. Ritchie, K. Murase, K. Suzuki, H. Murakoshi, R. S. Kasai, J. Kondo, and T. Fujiwara. Paradigm shift of the plasma membrane concept from the twodimensional continuum fluid to the partitioned fluid: high-speed single-molecule tracking of membrane molecules. Annu. Rev. Biophys. Biomol. Struct., 34:351-378, 2005.

[12] B. Christoffer Lagerholm, Gabriel E. Weinreb, Ken Jacobson, and Nancy L. Thompson. Detecting microdomains in intact cell membranes. Annual Review of Physical Chemistry, 56(1):309-336, 2005.

[13] D. A. Lauffenburger and J. Linderman. Receptors: models for binding, trafficking, and signaling. Oxford University Press, 1993.

[14] M. A. Lemon and J. Schlessinger. Cell signaling by receptor tyrosine kinases. Cell, 7(141):1117-1134, June 252010.

[15] D. S. Lidke and B. S. Wilson. Caught in the act: quanti- fying protein behaviour in living cells. Trends Cell Biol., 19(11):566-574, 2009.

[16] B. F. Lillemeier, J. R. Pfeiffer, Z. Surviladze, B. S. Wilson, and M. M. Davis. Plasma membrane-associated proteins are clustered into islands attached to the cytoskeleton. Proc Natl Acad Sci USA, 103:18992-7, 2006.

[17] D. Lingwood and K. Simons. Lipid rafts as a membrane-organizing principle. Science, 327(5961):46-50, 2010.

[18] M.M.Pryor, M. P. Steinkamp, A. M. Halasz, Y. Chen, S. Yang, M. S. Smith, G.ZahoranskyKohalmi, M. Swift, X. Xu, D. Hanien, et al. Orchestration of erbb3 signaling through heterointeractions and homointeractions. Molecular biology of the cell, 26(22):4109-4123, 2015.

[19] P. Nagy, G. Vereb, Z. Sebestyén, G. Horváth, S. J. Lockett, S. Damjanovich, J. W. Park, T. M. Jovin, and J. Szöllősi. Lipid rafts and the local density of erbb proteins influence the biological role of homo-and heteroassociations of erbb2. Journal of cell science, 115(22):42514262, 2002.

[20] D. A. Olsson, J. Kreuger, and L. Claesson-Welsh. Vegf receptor signaling - in control of vascular function. Nat Rev Mol Cell Biol, 7:359-371, 2006.

[21] J. R. Pfeiffer, J. M. Oliver, and B. S. Wilson. Observing signal transduction, endocytosis, and degranulation by immunogold labeling and transmission electron microscopy on membrane sheets. American biotechnology laboratory, 20(6):18-22, 2002.

[22] A. A. Philimonenko, P. Hozák, et al. Statistical evaluation of colocalization patterns in immunogold labeling experiments. Journal of structural biology, 132(3):201-210, 2000. 
[23] I. A. Prior, C. Muncke, R. G. Parton, and J. F. Hancock. Direct visualization of ras proteins in spatially distinct cell surface microdomains. The Journal of cell biology, 160(2):165-170, 2003.

[24] K. Radhakrishnan, Á. Halász, M. M. McCabe, J. S. Edwards, and B. S. Wilson. Mathematical simulation of membrane protein clustering for efficient signal transduction. Annals of biomedical engineering, 40(11):2307-2318, 2012.

[25] K. G. Rothberg, Y. Ying, and B. A. Kamen. Cholesterol controls the clustering of the glycophospholipid-anchored membrane receptor for 5-methyltetrahydrofolate. J. Cell Biology, 111:2931-2938, December 1990.

[26] C. A. Schneider, W.S. Rasband, and K.W. Eliceiri. Nih image to imagej: 25 years of image analysis. Nature Methods, 9:671-675, 2012.

[27] M. Skobe, T. Hawighorst, D. G. Jackson, R. Prevo, L. Janes, P. Velasco, L. Riccardi, K. Alitalo, K. Claffey, and M. Detmar. Induction of tumor lymphangiogenesis by vegf-c promotes breast cancer metastasis. Nature medicine, 7(2):192-198, 2001.

[28] S. A. Stacker, C. Caesar, M. E. Baldwin, G. E. Thornton, R. A. Williams, R. Prevo, D. G. Jackson, S. Nishikawa, H. Kubo, and M. G. Achen. Vegf-d promotes the metastatic spread of tumor cells via the lymphatics. Nature medicine, 7(2):186-191, 2001.

[29] K. Suzuki, K. Ritchie, E. Kajikawa, T. Fujiwara, and A. Kusumi. Rapid hop diffusion of a gprotein-coupled receptor in the plasma membrane as revealed by single-molecule techniques. Biophysical journal, 88(5):3659-3680, 2005.

[30] N. P. Wells, G. A. Lessard, P. M. Goodwin, M. E. Phipps, P. J. Cutler, D. S. Lidke, B. S. Wilson, and J. H. Werner. Time-resolved three-dimensional molecular tracking in live cells. Nano letters, 10(11):4732-4737, 2010.

[31] B. S. Wilson, J. M. Oliver, and D. S. Lidke. Spatio-temporal signaling in mast cells. In Mast Cell Biology, pages 91-106. Springer, 2011.

[32] B. S. Wilson, J. R. Pfeiffer, M. A. Raymond-Stintz, D. Lidke, and N. Andrews. Exploring membrane domains using native membrane sheets and transmission electron microscopy. Methods Mol Biol, 398:245-261, 2007.

[33] B. S. Wilson, J. R. Pfeiffer, M. A. Raymond-Stintz, D. Lidke, N. Andrews, J. Zhang, W. Yin, S. Steinberg, and J. M. Oliver. Exploring membrane domains using native membrane sheets and transmission electron microscopy. In Lipid Rafts, pages 245-261. Springer, 2007.

[34] B. S. Wilson, S. L. Steinberg, K. Liederman, J. R. Pfeiffer, Z. Surviladze, J. Zhang, L. E. Samelson, L. Yang, P. G. Kotula, and J. M. Oliver. Markers for detergent-resistant lipid rafts 
occupy distinct and dynamic domains in native membranes. Molecular biology of the cell, 15(6):2580-2592, 2004.

[35] J. Zhang, K. Leiderman, J. R. Pfeiffer, B. S. Wilson, J. M. Oliver, and S. L. Steinberg. Characterizing the topography of membrane receptors and signaling molecules from spatial patterns obtained using nanometer-scale electron-dense probes and electron microscopy. Micron, 37:14-34, 2006. 